\title{
Conformal geodesics in spherically symmetric vacuum spacetimes with Cosmological constant
}

\author{
A. García-Parrado Gómez-Lobo ${ }^{*, 1,3}$, E. Gasperín ${ }^{\dagger, 2}$, and J.A. Valiente Kroon ${ }^{\ddagger}, 2$ \\ ${ }^{1}$ Física Teórica, Universidad del País Vasco, Apartado 644, 48080 Bilbao, Spain. \\ ${ }^{2}$ School of Mathematical Sciences, Queen Mary, University of London, Mile End Road, London E1 4NS, \\ United Kingdom. \\ ${ }^{3}$ Centro de Matemática, Universidade do Minho, 4710-057 Braga, Portugal.
}

October 17, 2018

\begin{abstract}
An analysis of conformal geodesics in the Schwarzschild-de Sitter and Schwarzschild-anti de Sitter families of spacetimes is given. For both families of spacetimes we show that initial data on a spacelike hypersurface can be given such that the congruence of conformal geodesics arising from this data cover the whole maximal extension of canonical conformal representations of the spacetimes without forming caustic points. For the Schwarzschildde Sitter family, the resulting congruence can be used to obtain global conformal Gaussian systems of coordinates of the conformal representation. In the case of the Schwarzschild-anti de Sitter family, the natural parameter of the curves only covers a restricted time span so that these global conformal Gaussian systems do not exist.
\end{abstract}

\section{Contents}

1 Introduction

2 The Schwarzschild-de Sitter and Schwarzschild-anti de Sitter spacetimes 3

2.1 Specific properties of the Schwarzschild-de Sitter spacetime . . . . . . . . . 5

2.2 Specific properties of the Schwarzschild anti de Sitter spacetime . . . . . . . . 5

2.3 Penrose diagrams . . . . . . . . . . . . . . . . . . . 6

3 Conformal geodesics in vacuum spacetimes with Cosmological constant 6

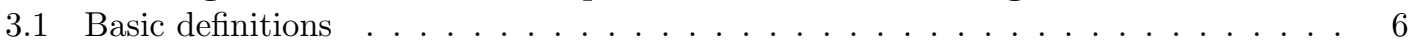

3.2 Conformal factors along conformal geodesics . . . . . . . . . . . . . . 7

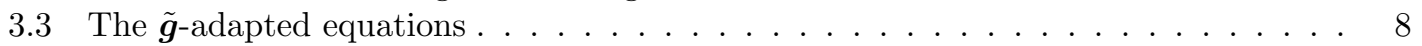

3.4 The deviation equations . . . . . . . . . . . . . . . . . . . 9

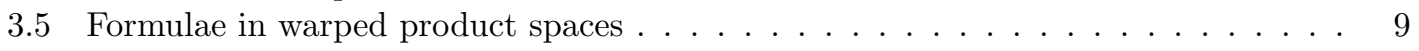

3.6 Explicit expressions for the reduced conformal geodesic equations . . . . . . . . . 10

3.7 Explicit expressions for the reduced conformal deviation equations . . . . . . . . . 12

4 Analysis of the conformal geodesics in the subextremal Schwarzschild-de Sitter case

$4.1 \quad$ Basic setup . . . . . . . . . . . . . . . . . . . . 12

4.2 Qualitative analysis of the behaviour of the curves . . . . . . . . . . . 14 4.2.1 Conformal geodesics with constant $r$ (critical curve).

\footnotetext{
*E-mail address:alfonso@math.uminho.pt

$\dagger$ E-mail address:e.gasperingarcia@qmul.ac.uk

$\ddagger$ E-mail address:j.a.valiente-kroon@qmul.ac.uk
} 
4.2.2 Conformal geodesics with $r_{\circledast}<r_{\star} \leq r_{c} \ldots \ldots \ldots \ldots$

4.2.3 Conformal geodesics with $r_{b} \leq r_{*}<r_{\circledast} \ldots \ldots \ldots \ldots$

4.2.4 Conformal geodesics through the bifurcation sphere of the cosmological

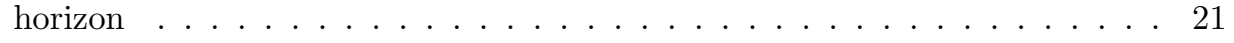

4.2.5 Conformal geodesic through the bifurcation sphere of the black hole horizon 23

4.3 Explicit expressions in terms of elliptic functions . . . . . . . . . . . . . . 24

4.4 Analysis of the behaviour of the conformal deviation equations . . . . . . . . . . 24

4.4.1 Conformal geodesics with $r_{\star}=r_{\circledast} \ldots \ldots \ldots \ldots$. . . . . . . . . . . . . . . .

4.4.2 Conformal geodesics with $r_{\star} \neq r_{\circledast} \ldots \ldots \ldots \ldots \ldots$

4.5 Conformal Gaussian coordinates in the subextremal Schwarzschild-de Sitter space-

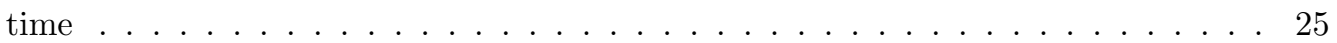

4.6 Summary of the analysis . . . . . . . . . . . . . . . . 27

5 Analysis of the conformal geodesics in the extremal

Schwarzschild-de Sitter case $\quad \underline{28}$

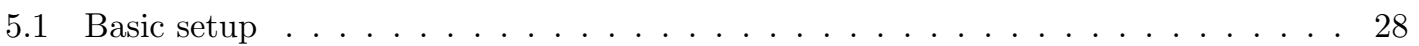

5.1 Conformal factor . . . . . . . . . . . . . . . . . 28

5.1 .2 Initial conditions for the congruence . . . . . . . . . . . . . . . 29

5.2 Qualitative analysis of the behaviour of the curves . . . . . . . . . . . . . . 30

5.2.1 Conformal geodesics with constant $t \ldots \ldots \ldots$. . . . . . . . . . . . . . . . .

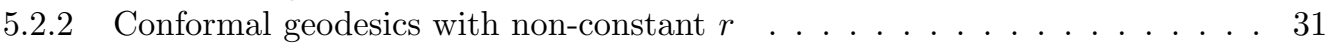

5.2.3 Behaviour of the congruence using null coordinates . . . . . . . . . . . . . 33

5.3 Explicit expressions in terms of elliptic functions . . . . . . . . . . . . . . . . 33

5.4 Analysis of the conformal geodesic deviation equation . . . . . . . . . . . 34

5.4 .1 Initial data for the deviation equation . . . . . . . . . . . . 34

5.4.2 Estimating the solution to the deviation equation . . . . . . . . . . . 35

5.5 Conformal Gaussian coordinates in the extremal Schwarzschild-de Sitter spacetime 35

5.6 Summary of the analysis . . . . . . . . . . . . . . . . . . 36

6 The Schwarzschild-anti de Sitter spacetime 36

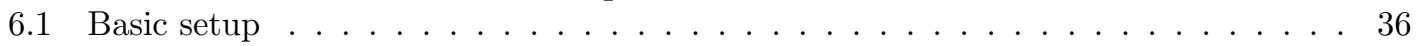

6.1 .1 Initial data . . . . . . . . . . . . . . . . . . . . . . . . . . .

6.1 .2 Technical observations . . . . . . . . . . . . . . . . 38

6.2 Qualitative analysis of the behaviour of the curves . . . . . . . . . . . . . 39

6.2.1 Conformal geodesics entering the horizon . . . . . . . . . . . . . 40

6.2 .2 Critical conformal geodesic . . . . . . . . . . . . . . . . 40

6.2.3 Conformal geodesics not entering the horizon . . . . . . . . . . . . . 42

6.2.4 Conformal geodesic starting at the bifurcation sphere . . . . . . . . . . 43

6.2.5 Conformal geodesics at the conformal boundary . . . . . . . . . . . . . 46

6.3 Analysis of the conformal geodesic deviation equation . . . . . . . . . . . . . . 48

6.4 Conformal Gaussian coordinates in the Schwarzschild-anti de Sitter spacetime . . . 49

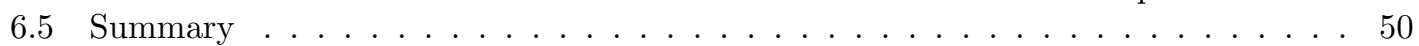

7 Concluding remarks

\section{Introduction}

The purpose of this article is to analyse the behaviour of conformal geodesics in vacuum spherically symmetric spacetimes with a Cosmological constant. Conformal geodesics are a powerful tool for the analysis of global properties of spacetimes. In addition to their conformal invariance, their relevance stems from the fact that they single out privileged representatives of the conformal class of a solution to the Einstein field equations. General properties of conformal geodesics in the context of General Relativity have been studied in [7, 6]. In particular, in the later reference it has been shown that for the Schwarzschild spacetime it is possible to construct a non-singular congruence of conformal geodesics covering the whole of the Kruskal-Székeres maximal extension 
of the spacetime. This congruence can be used, in turn, to construct a conformal Gaussian gauge system consisting of a system of coordinates and an adapted frame which are suitably propagated off a fiduciary hypersurface in the spacetime. The construction and analysis of the properties of this class of gauge systems can be regarded as a basic first step towards the analysis, by means of conformal methods, of generic spacetimes with a global structure similar to that of the Schwarzschild spacetime. The use of conformal Gaussian systems in conjunction with the conformal Einstein field equations renders a particularly attractive system of evolution equations for which all the conformal fields, save for the Weyl tensor, satisfy mere transport equations along the conformal geodesics - see e.g. 4, 5, 17.

The main result of our analysis is that, as in the case of the Schwarzschild spacetime, it is possible to construct congruences of conformal geodesics covering the maximal extensions of the subextremal and extremal Schwarzschild-de Sitter (SdS) solutions. These congruences allow, in turn, to construct global systems of conformal Gaussian coordinates. For the case of the Schwarzschild-anti de Sitter (SadS) spacetime, the situation is more subtle: although the congruence of conformal geodesics covers the whole of the maximal extension of the spacetime, the natural parameter of the curves of the congruence only describes a portion of the time span of the curves. A similar phenomenon has been observed in the anti de Sitter spacetime - see [4].

Applications of the constructions described in this article to the analysis of more general (i.e. non-symmetric) classes of vacuum spacetimes in the case of a de Sitter-Like Cosmological constant are given elsewhere - see 8 .

\section{Notations and conventions}

In what follows $a, b, c \ldots$ will denote spacetime abstract tensorial indices, while $i, j, k, \ldots$ are spatial tensorial indices ranging from 1 to 3 . By contrast, $\mu, \nu, \lambda, \ldots$ and $\alpha, \beta, \gamma, \ldots$ will correspond, respectively, to spacetime and spatial coordinate indices.

The signature convention for spacetime metrics is $(+,-,-,-)$. Thus, the induced metrics on spacelike hypersurfaces are negative definite.

An index-free notation will be often used. Given a 1-form $\boldsymbol{\omega}$ and a vector $\boldsymbol{v}$, we denote the action of $\boldsymbol{\omega}$ on $\boldsymbol{v}$ by $\langle\boldsymbol{\omega}, \boldsymbol{v}\rangle$. Furthermore, $\boldsymbol{\omega}^{\sharp}$ and $\boldsymbol{v}^{b}$ denote, respectively, the contravariant version of $\boldsymbol{\omega}$ and the covariant version of $\boldsymbol{v}$ (raising and lowering of indices) with respect to a given Lorentzian metric. This notation can be extended to tensors of higher rank (raising and lowering of all the tensorial indices). The conventions for the curvature tensors will fixed by the relation

$$
\left(\nabla_{a} \nabla_{b}-\nabla_{b} \nabla_{a}\right) v^{c}=R_{d a b}^{c} v^{d} .
$$

\section{The Schwarzschild-de Sitter and Schwarzschild-anti de Sitter spacetimes}

In the remaining of this article we will be concerned with the analysis of spherically symmetric spacetimes $(\tilde{\mathcal{M}}, \tilde{\boldsymbol{g}})$ satisfying the vacuum Einstein equations with Cosmological constant $\lambda$

$$
\tilde{R}_{a b}-\frac{1}{2} \tilde{R} \tilde{g}_{a b}-\lambda \tilde{g}_{a b}=0 .
$$

In the previous expression, $\tilde{R}_{a b}$ and $\tilde{R}$ denote, respectively, the Ricci tensor and the Ricci scalar of the metric $\tilde{\boldsymbol{g}}$. It follows that

$$
\tilde{R}_{a b}=-\lambda \tilde{g}_{a b}, \quad \tilde{R}=-4 \lambda .
$$

The minus sign in front of the Cosmological constant in equation (1) has been chosen to ensure that $\lambda>0$ corresponds to de Sitter-like spacetimes while $\lambda<0$ is associated to anti de Sitterlike spacetimes. The assumption of spherical symmetry dramatically reduces the number of solutions to the field equations (1). Indeed, a generalisation of Birkhoff's theorem shows that the 
only spherically symmetric solutions to the vacuum Einstein field equations with Cosmological constant are the Schwarzschild-de Sitter, the Schwarzschild-anti de Sitter and the Nariai solutions - see [16].

The line element of the Schwarzschild-de Sitter and Schwarzschild-anti de Sitter spacetimes is given, in standard coordinates $(t, r, \theta, \varphi)$, by

$$
\tilde{\boldsymbol{g}}=\left(1-\frac{2 m}{r}-\frac{\lambda}{3} r^{2}\right) \mathbf{d} t \otimes \mathbf{d} t-\left(1-\frac{2 m}{r}-\frac{\lambda}{3} r^{2}\right)^{-1} \mathbf{d} r \otimes \mathbf{d} r-r^{2} \boldsymbol{\sigma}
$$

where

$$
\boldsymbol{\sigma} \equiv\left(\mathbf{d} \theta \otimes \mathbf{d} \theta+\sin ^{2} \theta \mathbf{d} \varphi \otimes \mathbf{d} \varphi\right)
$$

is the standard metric of $\mathbb{S}^{2}$ and

$$
t \in(-\infty, \infty), \quad r \in(0, \infty), \quad \theta \in[0, \pi], \quad \varphi=[0,2 \pi) .
$$

In the conventions used in this article, the case $\lambda>0$ corresponds to the Schwarzschild-de Sitter spacetime and the case $\lambda<0$ to the Schwarzschild-anti de Sitter spacetime. In what follows, to simplify the computations we perform a rescaling of the line element in (2) so as to obtain the expression

$$
\tilde{\boldsymbol{g}}=\left(1-\frac{M}{r}+\epsilon r^{2}\right) \mathbf{d} t \otimes \mathbf{d} t-\left(1-\frac{M}{r}+\epsilon r^{2}\right)^{-1} \mathbf{d} r \otimes \mathbf{d} r-r^{2} \boldsymbol{\sigma}, \quad M \equiv 2 m \sqrt{\frac{-\epsilon \lambda}{3}} .
$$

The constant $\epsilon$ takes the value -1 for Schwarzschild-de Sitter case and +1 for the Schwarzschildanti de Sitter case - hence, $M$ is always kept strictly positive. It is convenient to define

$$
D(r) \equiv 1-\frac{M}{r}+\epsilon r^{2}=\frac{1}{r}\left(\epsilon r^{3}+r-M\right) .
$$

Note that in the representation given by the line element (3) both $M$ and $r$ are dimensionless quantities - this corresponds to a choice of units in which $\lambda=-3 \epsilon$, hence the Cosmological constant is also dimensionless.

In the sequel, it will be necessary to make use of alternative coordinate systems for the metric $\tilde{\boldsymbol{g}}$. In particular, an isotropic radial coordinate $\rho$ can be introduced by means of the relations

$$
r=r(\rho), \quad r^{\prime}(\rho)=\frac{r \sqrt{D(r)}}{\rho}
$$

so that the metric line element in equation 3 transforms into

$$
\tilde{\boldsymbol{g}}=D(r) \mathbf{d} t \otimes \mathbf{d} t-\frac{r^{2}}{\rho^{2}}\left(\mathbf{d} \rho \otimes \mathbf{d} \rho+\rho^{2} \boldsymbol{\sigma}\right) .
$$

The explicit form of the function $r(\rho)$ is not needed in our computations. In addition, we also make use of Eddington-Finkelstein null coordinates defined by the relations

$$
u=t-r, \quad v=t+r
$$

where $r$ is the tortoise coordinate defined via

$$
r(r)=\int \frac{\mathrm{d} r}{D(r)}
$$

the constant of integration in the last expression is usually to be chosen so that

$$
\lim _{r \rightarrow \infty} r(r)=0
$$

consequently $u, v \in \mathbb{R}$. Following standard conventions we refer to $u$ as the retarded null coordinate while $v$ is the advanced null coordinate. These coordinates render the line elements

$\tilde{\boldsymbol{g}}=D(r) \mathbf{d} u \otimes \mathbf{d} u-(\mathbf{d} u \otimes \mathbf{d} r+\mathbf{d} r \otimes \mathbf{d} u)-r^{2} \boldsymbol{\sigma}, \quad \tilde{\boldsymbol{g}}=D(r) \mathbf{d} v \otimes \mathbf{d} v+(\mathbf{d} v \otimes \mathbf{d} r+\mathbf{d} r \otimes \mathbf{d} v)-r^{2} \boldsymbol{\sigma}$. 


\subsection{Specific properties of the Schwarzschild-de Sitter spacetime}

In this Section the Schwarzschild-de Sitter case is analysed, consequently $\lambda>0$ will be assumed. Observe that, for $0<M<2 /(3 \sqrt{3})$, the function $D(r)$ can be rewritten as

$$
D(r)=-\frac{1}{r}\left(r-r_{b}\right)\left(r-r_{c}\right)\left(r-r_{-}\right)
$$

where $r_{b}$ and $r_{c}$ are two different positive real roots, and a $r_{-}$is a negative real. Moreover, one has that

$$
0<r_{b}<r_{c}, \quad r_{c}+r_{b}+r_{-}=0 .
$$

The root $r_{b}$ corresponds to a black hole-type horizon, while the root $r_{c}$ is associated to a Cosmological de Sitter-like horizon. It can be readily verified that $D(r)>0$ for $r_{b}<r<r_{c}$ while $D(r)<0$ for $0<r<r_{b}$ and $r>r_{c}$. Consequently, the metric is static in the region $r_{b}<r<r_{c}$ between the two horizons and there are no other static regions outside this range for $r$. Using Cardano's formula for cubic equations it is possible to find the explicit values of $r_{b}, r_{c}$ and $r_{-}$. One finds that

$$
\begin{aligned}
& r_{-}=-\frac{2}{\sqrt{3}} \cos \left(\frac{\phi}{3}\right), \\
& r_{b}=\frac{1}{\sqrt{3}}\left(\cos \left(\frac{\phi}{3}\right)-\sqrt{3} \sin \left(\frac{\phi}{3}\right)\right), \\
& r_{c}=\frac{1}{\sqrt{3}}\left(\cos \left(\frac{\phi}{3}\right)+\sqrt{3} \sin \left(\frac{\phi}{3}\right)\right),
\end{aligned}
$$

where the parameter $\phi$ is defined through the relation

$$
M=\frac{2 \cos \phi}{3 \sqrt{3}}, \quad \phi \in\left(0, \frac{1}{2} \pi\right) .
$$

We will refer to this case, for which $\phi \in\left(0, \frac{1}{2} \pi\right)$, as the subextremal Schwarzschild de-Sitter spacetime. In contrast, the case $\phi=\pi / 2$ for which $M=2 /(3 \sqrt{3})$ will be referred as the extremal Schwarzschild de-Sitter spacetime. This is of special interest as one has

$$
r_{\mathcal{H}} \equiv r_{b}=r_{c}=\frac{3 M}{2}=\frac{1}{\sqrt{3}}
$$

so that the function $D(r)$ takes the form

$$
D(r)=-\frac{1}{r}\left(\frac{2}{\sqrt{3}}+r\right)\left(r-\frac{1}{\sqrt{3}}\right)^{2} .
$$

Finally, it is observed that one can also consider the hyperextremal Schwarzschild de-Sitter spacetime characterised by the condition $M>2 /(3 \sqrt{3})$. In this case, the spacetime does not contain horizons.

\subsection{Specific properties of the Schwarzschild anti de Sitter spacetime}

For the Schwarzschild-anti de Sitter spacetime $\lambda<0$, consequently, in this case, the function $D(r)$ has a single real root $r_{b}$. Using Cardano's formula one finds that

$$
r_{b}=\frac{2}{\sqrt{3}} \sinh \frac{\phi}{3}, \quad M=\frac{2 \sinh \phi}{3 \sqrt{3}}, \quad \phi \in(0, \infty) .
$$

The root $r_{b}$ is associated to an event horizon separating two regions: a black hole region with a singularity at $r=0$ and an exterior region defined by the condition $r>r_{b}$ where the spacetime is static - see Figure 3 . One can eliminate the parameter $\phi$ in equation $(13)$ and write $M$ in terms of $r_{b}$ as

$$
M=r_{b}+r_{b}^{3}
$$




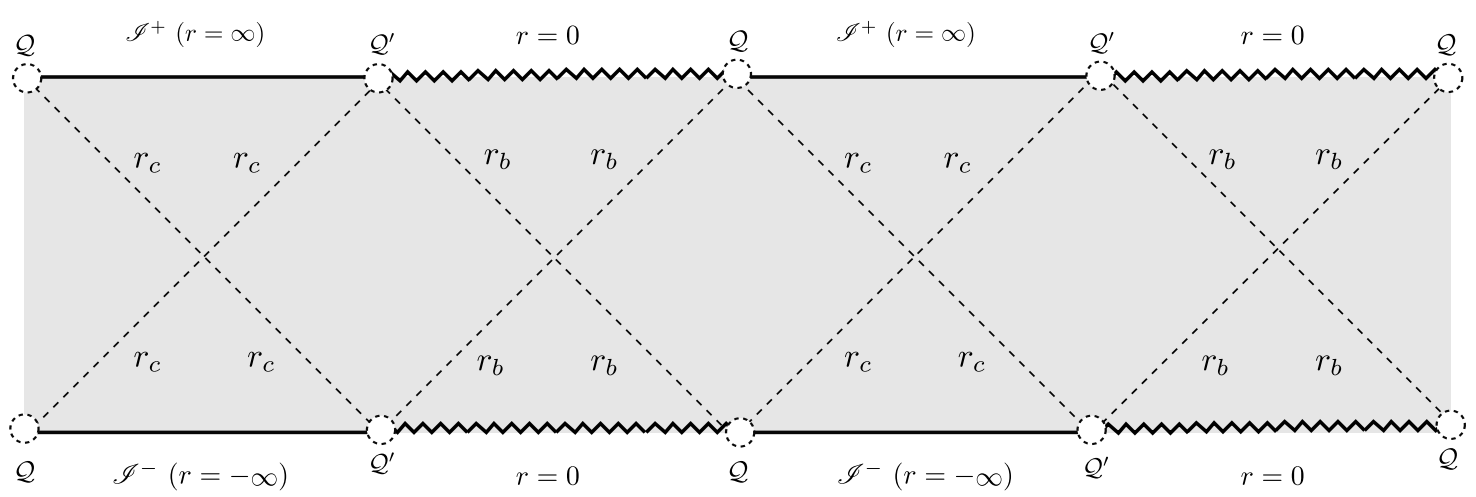

Figure 1: Penrose diagram for the subextremal Schwarzschild-de Sitter spacetime. The serrated line denotes the location of the singularity; the continuous black line denotes the conformal boundary; the dashed line shows the location of the black hole and cosmological horizons which are located at $r=r_{b}$ and $r=r_{c}$ respectively. The excluded points $\mathcal{Q}$ and $\mathcal{Q}^{\prime}$ where the singularity seems to meet the conformal boundary correspond to asymptotic regions of the spacetime that does not belong to the singularity nor the conformal boundary.

\subsection{Penrose diagrams}

The Penrose diagrams of the Schwarzschild-de Sitter and the Schwarzschild-anti de Sitter spacetimes are well known - see e.g., 9] for details on their construction. These diagrams are given in Figures 1 1 . A discussion of the general procedure for the construction of Penrose diagrams in spherically symmetric spacetimes can be found in [17, Chapter 6 .

\section{Conformal geodesics in vacuum spacetimes with Cosmo- logical constant}

In this Section some basic results concerning conformal geodesics in vacuum spacetimes are briefly reviewed. Full details can be found in [4, 6, 14] - see also [17]. In what follows, let $(\tilde{\mathcal{M}}, \tilde{\boldsymbol{g}})$ denote a spacetime satisfying the vacuum Einstein field equations with Cosmological constant (1).

\subsection{Basic definitions}

Given an interval $I \subseteq \mathbb{R}$, let $x(\tau), \tau \in I$ denote a curve in $(\mathcal{M}, \tilde{\boldsymbol{g}})$ and let $\boldsymbol{\beta}(\tau)$ denote a 1 -form along $\boldsymbol{x}(\tau)$. Furthermore, let $\dot{x} \equiv \mathrm{d} \boldsymbol{x} / \mathrm{d} \tau$ denote the tangent vector field of the curve $x(\tau)$. The conformal geodesic equations are then given by:

$$
\begin{aligned}
\tilde{\nabla}_{\dot{\boldsymbol{x}}} \dot{\boldsymbol{x}} & =-2\langle\boldsymbol{\beta}, \dot{\boldsymbol{x}}\rangle \dot{\boldsymbol{x}}+\tilde{\boldsymbol{g}}(\dot{\boldsymbol{x}}, \dot{\boldsymbol{x}}) \boldsymbol{\beta}^{\sharp}, \\
\tilde{\nabla}_{\dot{\boldsymbol{x}}} \boldsymbol{\beta} & =\langle\boldsymbol{\beta}, \dot{\boldsymbol{x}}\rangle \boldsymbol{\beta}-\frac{1}{2} \tilde{\boldsymbol{g}}^{\sharp}(\boldsymbol{\beta}, \boldsymbol{\beta}) \dot{\boldsymbol{x}}^{b}+\tilde{\boldsymbol{L}}(\dot{\boldsymbol{x}}, \cdot),
\end{aligned}
$$

where $\tilde{\nabla}$ denotes the Levi-Civita connection of the physical metric $\tilde{\boldsymbol{g}}$ and $\tilde{\nabla}_{\dot{\boldsymbol{x}}}$ denotes a derivative in the direction of $\dot{\boldsymbol{x}}$. Notice that in the last expression the indices of the vectors and covectors are raised or lowered using $\tilde{\boldsymbol{g}}$ - unless otherwise stated, we follow this convention in the rest of this article. The symbol $\tilde{\boldsymbol{L}}$ denotes the Schouten tensor of $\tilde{\boldsymbol{g}}$ defined by:

$$
\tilde{L}_{a b} \equiv \frac{1}{2}\left(\tilde{R}_{a b}-\frac{1}{6} \tilde{R} \tilde{g}_{a b}\right) .
$$

In the case of a spacetime satisfying the vacuum equations with Cosmological constant (1) one has that:

$$
\tilde{L}_{a b}=-\frac{1}{6} \lambda \tilde{g}_{a b}
$$

In the remainder of this article it is assumed that the spacetime $(\tilde{\mathcal{M}}, \tilde{\boldsymbol{g}})$ satisfies condition 16 . 


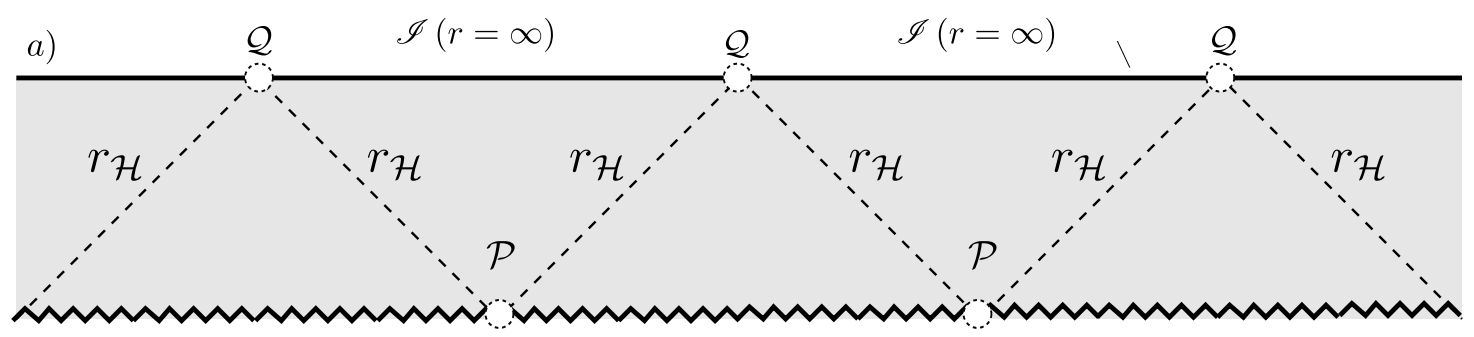

b)

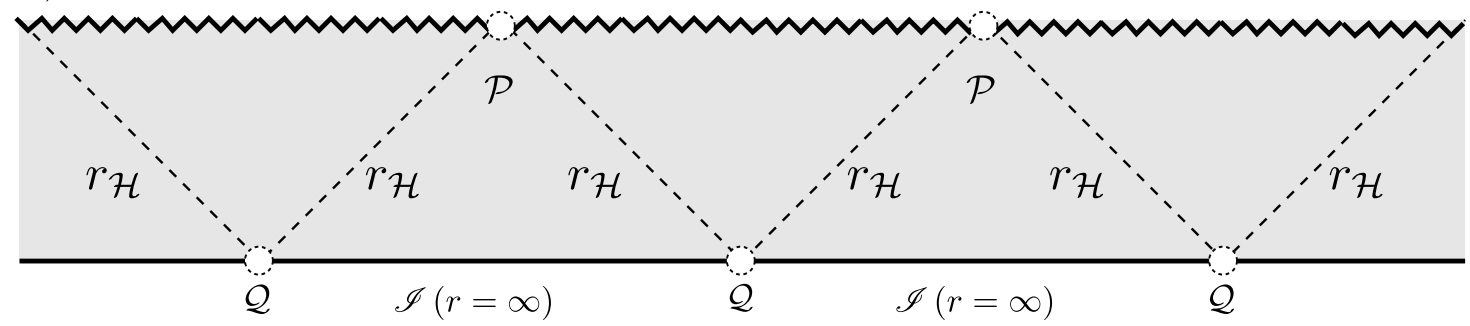

Figure 2: Penrose diagrams for the extremal Schwarzschild-de Sitter spacetime. Figure (a) corresponds to a white hole which evolves towards a de Sitter final state while Figure (b) is a model of a black hole with a future singularity. The Killing horizon is located at $r=r_{\mathcal{H}}$ as described in the main text. Similar to the subextremal case, the excluded points denoted by $\mathcal{P}, \mathcal{Q}$ represent asymptotic regions of the spacetime that do not belong to the singularity nor the conformal boundary.

\subsection{Conformal factors along conformal geodesics}

Conformal geodesics allow to single out a canonical representative of the conformal class of $\tilde{\boldsymbol{g}}$. Let $(\mathcal{M}, \boldsymbol{g})$ denote a conformal extension of $(\tilde{\mathcal{M}}, \tilde{\boldsymbol{g}})$. Hence, there exists a scalar $\Theta$ such that the metrics $\tilde{\boldsymbol{g}}$ and $\boldsymbol{g}$ are related via

$$
\boldsymbol{g}=\Theta^{2} \tilde{\boldsymbol{g}}
$$

The conformal factor $\Theta$ can be fixed by requiring $\boldsymbol{x}(\tau)$ to be timelike and imposing the normalisation condition

$$
\boldsymbol{g}(\dot{\boldsymbol{x}}, \dot{\boldsymbol{x}})=1 .
$$

Using equation (16), repeated differentiation of condition (18) together with the conformal geodesic equations and the Einstein field equations expressed as in equation 16 one obtains the relations

$$
\dot{\Theta}=\langle\boldsymbol{\beta}, \boldsymbol{x}\rangle \Theta, \quad \ddot{\Theta}=\Theta^{-1}\left(\frac{1}{2} \tilde{\boldsymbol{g}}^{\sharp}(\boldsymbol{\beta}, \boldsymbol{\beta})-\frac{1}{6} \lambda\right), \quad \dddot{\Theta}=0,
$$

where $\dot{\Theta} \equiv \tilde{\nabla}_{\dot{x}} \Theta$, etc. Integrating the last of these equations along a given conformal geodesic one finds

$$
\Theta=\Theta_{\star}+\dot{\Theta}_{\star}\left(\tau-\tau_{\star}\right)+\frac{1}{2} \ddot{\Theta}_{\star}\left(\tau-\tau_{\star}\right)^{2},
$$

where $\Theta_{\star}, \dot{\Theta}_{\star}$ and $\ddot{\Theta}_{\star}$ are prescribed at a fiduciary value $\tau_{\star}$ of the parameter along the conformal geodesic $x(\tau)$. The coefficients $\dot{\Theta}_{\star}$ and $\ddot{\Theta}_{\star}$ satisfy the constraints

$$
\dot{\Theta}_{\star}=\left\langle\boldsymbol{\beta}_{\star}, \dot{\boldsymbol{x}}_{\star}\right\rangle \Theta_{\star}, \quad \Theta_{\star} \ddot{\Theta}_{\star}=\frac{1}{2} \tilde{\boldsymbol{g}}\left(\boldsymbol{\beta}_{\star}, \boldsymbol{\beta}_{\star}\right)-\frac{1}{6} \lambda,
$$

where $\boldsymbol{\beta}_{\star}$ and $\dot{\boldsymbol{x}}_{\star}$ denote, respectively, the value of $\boldsymbol{\beta}$ and $\dot{\boldsymbol{x}}$ at $\tau=\tau_{\star}$. A further computation exploiting the above expressions shows that

$$
\tilde{\nabla}_{a} \Theta \tilde{\nabla}^{a} \Theta=\frac{1}{3} \lambda \text { at } \mathscr{I} .
$$

Thus, as it is well known for vacuum spacetimes, the causal character of the conformal boundary is determined by the sign of $\lambda$-spacelike if $\lambda>0$ and timelike if $\lambda<0$. 


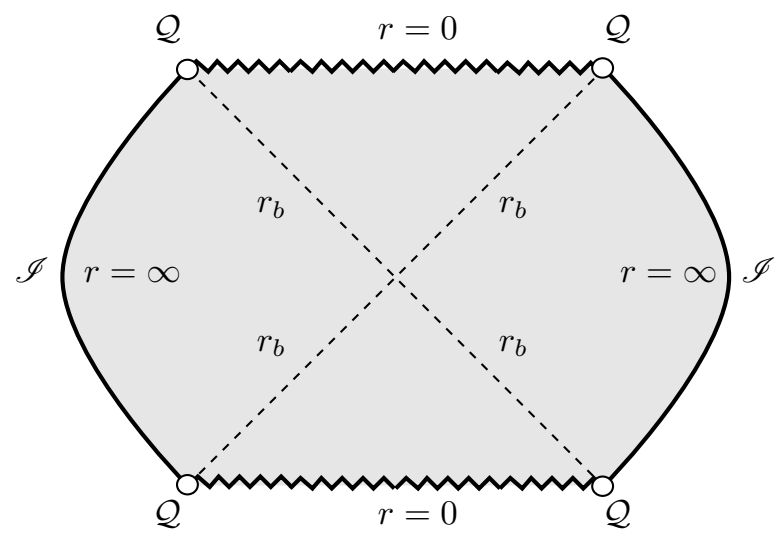

Figure 3: Penrose diagram of the Schwarzschild-anti de Sitter spacetime. Similar notation as that used for the Penrose diagrams of the Schwarzschild-de Sitter spacetime has been used. The black hole horizon is located at at $r=r_{b}$ and the asymptotic points are denoted by $\mathcal{Q}$. Notice that the singularity is of spacelike nature and, in contrast with the de-Sitter like case, the conformal boundary is timelike. Following the discussion in [11, 3], generically, if the singularity is represented as a straight horizontal line, the conformal boundary must be represented as curved. A more accurate representation of the singularity can be found in figures 10 and 11 .

Remark 1. Notice that by virtue of the normalisation condition $(18), \tau$ is the $\boldsymbol{g}$-proper time of the conformal geodesic.

\subsection{The $\tilde{g}$-adapted equations}

In specific computations, it is more convenient to consider a parametrisation of the conformal geodesics in terms of the physical proper time. To this end, consider the parameter transformation $\tilde{\tau}=\tilde{\tau}(\tau)$ given by

$$
\tilde{\tau}=\tilde{\tau}_{\star}+\int_{\tau_{\star}}^{\tau} \frac{\mathrm{d} s}{\Theta(s)},
$$

with inverse $\tau=\tau(\tilde{\tau})$. In what follows, let $\tilde{\boldsymbol{x}}(\tilde{\tau}) \equiv \boldsymbol{x}(\tau(\tilde{\tau}))$. Using this notation, it can then be verified that

$$
\tilde{\boldsymbol{x}}^{\prime} \equiv \frac{\mathrm{d} \tilde{x}}{\mathrm{~d} \tilde{\tau}}=\Theta \dot{\boldsymbol{x}}
$$

and that

$$
\tilde{\boldsymbol{g}}\left(\tilde{\boldsymbol{x}}^{\prime}, \tilde{\boldsymbol{x}}^{\prime}\right)=1,
$$

so that $\tilde{\tau}$ is the $\tilde{\boldsymbol{g}}$-proper time of the curve $\tilde{\boldsymbol{x}}$. In order to write the equation for the curve $\tilde{x}(\bar{\tau})$ in a convenient way, we consider the split

$$
\boldsymbol{\beta}=\tilde{\boldsymbol{\beta}}+\varpi \dot{\boldsymbol{x}}^{b},
$$

where the 1-form $\tilde{\boldsymbol{\beta}}$ satisfies

$$
\langle\tilde{\boldsymbol{\beta}}, \dot{\boldsymbol{x}}\rangle=0, \quad \varpi \equiv \frac{\langle\boldsymbol{\beta}, \dot{\boldsymbol{x}}\rangle}{\tilde{\boldsymbol{g}}(\dot{\boldsymbol{x}}, \dot{\boldsymbol{x}})}, \quad \boldsymbol{g}^{\sharp}(\boldsymbol{\beta}, \boldsymbol{\beta})=\langle\boldsymbol{\beta}, \dot{\boldsymbol{x}}\rangle^{2}+\boldsymbol{g}^{\sharp}(\tilde{\boldsymbol{\beta}}, \tilde{\boldsymbol{\beta}}) .
$$

Moreover one can verify that

$$
\tilde{\boldsymbol{g}}=\Theta^{-2} \boldsymbol{g}, \quad\langle\boldsymbol{\beta}, \dot{x}\rangle=\Theta^{-1} \dot{\Theta}, \quad \varpi=\Theta^{-1} \dot{\Theta} .
$$

In terms of these objects the $\tilde{\boldsymbol{g}}$-adapted equations for the conformal curves are given by

$$
\begin{aligned}
& \tilde{\nabla}_{\tilde{\boldsymbol{x}}^{\prime}} \tilde{\boldsymbol{x}}^{\prime}=\tilde{\boldsymbol{\beta}}^{\sharp}, \\
& \tilde{\nabla}_{\tilde{\boldsymbol{x}}^{\prime}} \tilde{\boldsymbol{\beta}}=\beta^{2} \tilde{\boldsymbol{x}}^{\prime b},
\end{aligned}
$$


where

$$
\beta^{2} \equiv-\tilde{\boldsymbol{g}}^{\sharp}(\tilde{\boldsymbol{\beta}}, \tilde{\boldsymbol{\beta}})
$$

is constant along a given conformal geodesic.

\subsection{The deviation equations}

When working with congruences of conformal geodesics it is important to analyse whether they develop conjugate points. To this end, let $x(\tau, \sigma)$ and $\boldsymbol{\beta}(\tau, \sigma)$ denote a family of conformal curves depending smoothly on a parameter $\sigma$. Following [6], let

$$
\boldsymbol{z} \equiv \partial_{\sigma} x, \quad \boldsymbol{\omega} \equiv \tilde{\nabla}_{\boldsymbol{z}} \boldsymbol{\beta} .
$$

The fields $\boldsymbol{z}$ and $\boldsymbol{\omega}$ denote, respectively, the deviation vector field and the deviation 1-form. The conformal Jacobi equation and the 1-form deviation equation are given by

$$
\begin{aligned}
& \tilde{\nabla}_{\dot{\boldsymbol{x}}} \tilde{\nabla}_{\dot{\boldsymbol{x}}} \boldsymbol{z}=\tilde{\boldsymbol{R}}(\dot{\boldsymbol{x}}, \boldsymbol{z}) \dot{\boldsymbol{x}}-\boldsymbol{S}(\boldsymbol{\omega} ; \dot{\boldsymbol{x}}, \dot{\boldsymbol{x}})-2 \boldsymbol{S}\left(\boldsymbol{\beta} ; \dot{\boldsymbol{x}}, \tilde{\nabla}_{\dot{\boldsymbol{x}}} \boldsymbol{z}\right), \\
& \tilde{\nabla}_{\dot{\boldsymbol{x}} \boldsymbol{\omega}}=-\boldsymbol{\beta} \cdot \tilde{\boldsymbol{R}}(\dot{\boldsymbol{x}}, \boldsymbol{z})+\frac{1}{2}\left(\boldsymbol{\omega} \cdot \boldsymbol{S}(\boldsymbol{\beta} ; \dot{\boldsymbol{x}}, \cdot)+\boldsymbol{\beta} \cdot \boldsymbol{S}(\boldsymbol{\omega} ; \dot{\boldsymbol{x}}, \cdot)+\boldsymbol{\beta} \cdot \boldsymbol{S}\left(\boldsymbol{\beta} ; \tilde{\nabla}_{\dot{\boldsymbol{x}}} \boldsymbol{z}, \cdot\right)\right),
\end{aligned}
$$

where $\tilde{\boldsymbol{R}}(\cdot, \cdot)$ denotes the Riemann tensor of the metric $\tilde{\boldsymbol{g}}$, and

$$
\begin{aligned}
& \boldsymbol{S}(\boldsymbol{\beta} ; \dot{\boldsymbol{x}}, \boldsymbol{y}) \equiv\langle\boldsymbol{\beta}, \dot{\boldsymbol{x}}\rangle \boldsymbol{y}+\langle\boldsymbol{\beta}, \boldsymbol{y}\rangle \dot{\boldsymbol{x}}-\tilde{\boldsymbol{g}}(\dot{\boldsymbol{x}}, \boldsymbol{y}) \boldsymbol{\beta}^{\sharp}, \\
& \boldsymbol{\omega} \cdot \boldsymbol{S}(\boldsymbol{\beta} ; \dot{\boldsymbol{x}}, \cdot) \equiv\langle\boldsymbol{\omega}, \dot{\boldsymbol{x}}\rangle \boldsymbol{\beta}+\langle\boldsymbol{\beta}, \dot{\boldsymbol{x}}\rangle \boldsymbol{\omega}-\tilde{\boldsymbol{g}}^{\sharp}(\boldsymbol{\omega}, \boldsymbol{\beta}) \dot{\boldsymbol{x}}^{\mathrm{b}} .
\end{aligned}
$$

A $\tilde{\boldsymbol{g}}$-adapted version of the conformal geodesics can be readily computed. Accordingly, let $\tilde{x} \equiv x(\tilde{\tau}, \sigma)$ be a reparametrisation of $x(\tau, \sigma)$ in terms of the physical proper time $\tilde{\tau}$. Moreover, let

$$
\tilde{\boldsymbol{z}} \equiv \partial_{\sigma} \tilde{x}, \quad \tilde{\boldsymbol{\omega}} \equiv \tilde{\nabla}_{\tilde{\boldsymbol{z}}} \tilde{\boldsymbol{\beta}} .
$$

In terms of these new variables one has that equations 27a)-27b take the form

$$
\begin{aligned}
& \tilde{\nabla}_{\tilde{\boldsymbol{x}}^{\prime}} \tilde{\nabla}_{\tilde{\boldsymbol{x}}^{\prime}} \boldsymbol{z}=\tilde{\boldsymbol{R}}\left(\tilde{\boldsymbol{x}}^{\prime}, \boldsymbol{z}\right) \tilde{\boldsymbol{x}}^{\prime}+\tilde{\boldsymbol{\omega}}^{\sharp}, \\
& \tilde{\nabla}_{\tilde{\boldsymbol{x}}^{\prime}} \tilde{\boldsymbol{\omega}}=-\tilde{\boldsymbol{\beta}} \cdot \tilde{\boldsymbol{R}}\left(\tilde{\boldsymbol{x}}^{\prime}, \tilde{\boldsymbol{z}}\right)+\tilde{\boldsymbol{x}}^{\prime b} \tilde{\nabla}_{\tilde{\boldsymbol{z}}} \beta^{2}+\beta^{2} \tilde{\nabla}_{\tilde{\boldsymbol{x}}^{\prime}} \tilde{\boldsymbol{z}}^{b} .
\end{aligned}
$$

A computation shows that

$$
\tilde{\nabla}_{\tilde{x}^{\prime}} \tilde{\nabla}_{\tilde{z}} \beta^{2}=0 .
$$

Therefore, $\tilde{\nabla}_{\tilde{z}} \beta^{2}$ is constant along a given conformal geodesic.

\subsection{Formulae in warped product spaces}

The line element (2) is in the form of a warped product. This structure can be exploited to simplify the analysis of the $\tilde{\boldsymbol{g}}$-adapted conformal geodesic equations (25a)-(25b). To have a selfcontained discussion, the main formulae for warped product spacetimes, originally derived in [6], are given in this Section.

In what follows, the discussion will be particularised to spacetimes whose metric can be written in the warped product form:

$$
\tilde{\boldsymbol{g}}=\tilde{l}_{A B} \mathbf{d} x^{A} \otimes \mathbf{d} x^{B}+f^{2} \tilde{k}_{i j} \mathbf{d} x^{i} \otimes \mathbf{d} x^{j},
$$

with

$$
\tilde{l}_{A B}=l_{A B}\left(x^{C}\right), \quad \tilde{k}_{i j}=k_{i j}\left(x^{k}\right), \quad f=f\left(x^{A}\right)>0,
$$

and $A, B, C=0,1$ and $i, j, k=2,3$. In addition, it is assumed that the 2-dimensional metric given by $\tilde{\boldsymbol{l}} \equiv \tilde{l}_{A B} \mathbf{d} x^{A} \otimes \mathbf{d} x^{B}$ is Lorentzian, while $\tilde{\boldsymbol{k}}=\tilde{k}_{i j} \mathbf{d} x^{i} \otimes \mathbf{d} x^{j}$ is a negative-definite 2dimensional Riemannian metric. In view of this structure it is natural to consider solutions to the conformal curve equations satisfying $\dot{x}^{i}=0$ and $b_{j}=0$. In the case of the metric given by the line element (2) this Ansatz leads to solutions to the conformal curves which have no evolution on the 
angular coordinates, and one has only to consider evolution equations for the coordinates $(t, r)$ - or alternatively $(u, r)$ or $(v, r)$. A direct computation shows that for this type of conformal geodesics the $\tilde{\boldsymbol{g}}$-adapted equations for the conformal geodesics imply

$$
\begin{aligned}
& \not D \tilde{\boldsymbol{x}}^{\prime} \tilde{\boldsymbol{x}}^{\prime}=\tilde{\boldsymbol{\beta}}^{\sharp}, \\
& \tilde{\boldsymbol{\beta}}= \pm \beta \tilde{\boldsymbol{\epsilon}}_{\boldsymbol{l}}\left(\tilde{\boldsymbol{x}}^{\prime}, \cdot\right),
\end{aligned}
$$

with

$$
\epsilon_{\tilde{l}} \equiv \sqrt{|\Delta|} \mathbf{d} x^{0} \wedge \mathbf{d} x^{1}, \quad \Delta \equiv \operatorname{det} \tilde{l}_{A B}
$$

denoting the volume form of $\tilde{\boldsymbol{l}}$ and where $\not D$ denotes the Levi-Civita covariant derivative of $\tilde{\boldsymbol{l}}$. A further computation shows that under the present Ansatz, the $\tilde{\boldsymbol{g}}$-adapted deviation equations 28a)-28b are equivalent to each other and to the equation

$$
\not \tilde{\boldsymbol{x}}^{\prime} \not D_{\tilde{\boldsymbol{x}}^{\prime}} \tilde{\boldsymbol{z}}=\frac{1}{2} R[\tilde{l}] \boldsymbol{\epsilon}_{\tilde{\boldsymbol{l}}}\left(\tilde{\boldsymbol{x}}^{\prime}, \tilde{\boldsymbol{z}}\right) \boldsymbol{\epsilon}_{\tilde{\boldsymbol{l}}}\left(\tilde{\boldsymbol{x}}^{\prime}, \cdot\right)^{\sharp} \pm\left(\not D_{\tilde{\boldsymbol{z}}} \beta \boldsymbol{\epsilon}_{\tilde{\boldsymbol{l}}}\left(\tilde{\boldsymbol{x}}^{\prime}, \cdot\right)^{\sharp}+\beta \boldsymbol{\epsilon}_{\tilde{\boldsymbol{l}}}\left(\not D_{\tilde{\boldsymbol{x}}^{\prime}} \tilde{\boldsymbol{z}}, \cdot\right)\right),
$$

where $R[\tilde{l} \tilde{l}]$ denotes the Ricci scalar of $\tilde{\boldsymbol{l}}$.

For conformal curves satisfying $\dot{x}^{a}=0$ and $b_{c}=0$, the question of whether the deviation vector field $\tilde{\boldsymbol{z}}$ is non-vanishing can be rephrased in terms of a similar question for the scalar

$$
\tilde{\omega} \equiv \boldsymbol{\epsilon}_{\tilde{l}}\left(\tilde{\boldsymbol{x}}^{\prime}, \tilde{\boldsymbol{z}}\right) .
$$

Notice that as long as $\tilde{\omega} \neq 0, \tilde{\boldsymbol{x}}^{\prime}$ and $\tilde{\boldsymbol{z}}$ are linearly independent. A computation using the deviation equation (31) yields

$$
\not D_{\tilde{\boldsymbol{x}}^{\prime}} \not_{\tilde{\boldsymbol{x}}^{\prime}} \tilde{\omega}=\left(\beta^{2}+\frac{1}{2} R[\tilde{\boldsymbol{l}}]\right) \tilde{\omega}+\not \tilde{\boldsymbol{z}}_{\tilde{z}} \beta .
$$

If we regard the congruence of conformal geodesics as a congruence in a conformal extension $(\mathcal{M}, \boldsymbol{g})$ with $\boldsymbol{g}=\Theta^{2} \tilde{\boldsymbol{g}}$ then the scalar which we need to analyse is

$$
\omega \equiv \epsilon_{l}(\dot{\boldsymbol{x}}, \boldsymbol{z})
$$

where $\boldsymbol{l} \equiv \Theta^{2} \tilde{\boldsymbol{l}}$. The scalar $\omega$ has a similar geometric meaning as $\tilde{\omega}$ for the congruence of conformal geodesics regarded as curves in the unphysical spacetime. From equation 222 we deduce that $\tilde{\boldsymbol{x}}^{\prime}=\Theta \dot{\boldsymbol{x}}$ and in addition $\boldsymbol{\epsilon}_{\boldsymbol{l}}=\Theta^{2} \boldsymbol{\epsilon}_{\tilde{\boldsymbol{l}}}$. Hence

$$
\omega=\Theta \tilde{\omega} .
$$

\subsection{Explicit expressions for the reduced conformal geodesic equations}

The corresponding 2-dimensional metric $\boldsymbol{l}$, as determined by the warped product form 29], for the line element of equation (3), is given by

$$
\tilde{l}=D(r) \mathbf{d} t \otimes \mathbf{d} t-D^{-1}(r) \mathbf{d} r \otimes \mathbf{d} r .
$$

It follows that the reduced conformal geodesic equations (30a)-30b reduce to

$$
\begin{aligned}
& t^{\prime \prime}+\frac{\partial_{r} D(r)}{D(r)} r^{\prime} t^{\prime}=\frac{1}{D(r)} \beta r^{\prime}, \\
& r^{\prime \prime}-\frac{1}{2} \frac{\partial_{r} D(r)}{D(r)} r^{\prime 2}+\frac{1}{2} D(r) \partial_{r} D(r) t^{\prime 2}=D(r) \beta t^{\prime},
\end{aligned}
$$

where consistent with the notation of Section 3.3 we have set $r \equiv r(\tilde{\tau})$ and $t \equiv t(\tilde{\tau})$. Initial data for these equations can be prescribed following the discussion of Sections $3.1[3.3$. Observe that equations 37a-37b can be decoupled by making use of the $\tilde{\boldsymbol{g}}$-normalisation condition

$$
D(r) t^{\prime 2}-\frac{1}{D(r)} r^{\prime 2}=1 .
$$


Solving the latter for $t^{\prime} \geq 0$ and substituting into equation $37 \mathrm{~b}$, one obtains that

$$
r^{\prime \prime}+\frac{1}{2} \partial_{r} D(r)-\beta \sqrt{D(r)+r^{\prime 2}}=0 .
$$

This equation can be integrated once to yield

$$
\sqrt{D(r)+r^{\prime 2}}-\beta r=\gamma,
$$

where $\gamma$ is a constant given in terms of the initial value of the radial coordinate $r_{\star}$ by

$$
\gamma \equiv-\beta r_{\star}+\sqrt{D_{\star}+r_{\star}^{\prime}}, \quad D_{\star} \equiv D\left(r_{\star}\right)>0, \quad r_{\star}^{\prime} \equiv r^{\prime}\left(\tilde{\tau}_{\star}\right) .
$$

It follows that

$$
r^{\prime}= \pm \sqrt{(\gamma+\beta r)^{2}-D(r)},
$$

with the sign depending on the value of $r_{\star}$. Substituting the latter expression into equation (38) we get

$$
t^{\prime}=\frac{|\gamma+\beta r|}{|D(r)|} .
$$

One can formally integrate equations 44 and 42 as

$$
\begin{aligned}
& \tilde{\tau}(r)-\tilde{\tau}_{\star}=\int_{r_{\star}}^{r} \frac{1}{\sqrt{(\gamma+\beta \bar{r})^{2}-D(\bar{r})}} \mathrm{d} \bar{r}, \\
& t(r)-t_{\star}=\int_{r_{\star}}^{r} \frac{|\gamma+\beta \bar{r}|}{\sqrt{\left(\gamma^{2}+\beta \bar{r}\right)^{2}-D(\bar{r})}} \mathrm{d} \bar{r} .
\end{aligned}
$$

Remark 2. As a notational remark we stress that $\bar{r}$ is only used as a variable for integration. Notice, in contrast, that $\tau$ and $\tilde{\tau}$ represent the $\boldsymbol{g}$ and $\tilde{\boldsymbol{g}}$ proper time.

The solutions of equations (41) and (42), or equivalently expressions 43a and (43b), completely determine $r=r\left(\tilde{\tau}, r_{\star}\right)$ and $t=t\left(\tilde{\tau}, r_{\star}\right)$ which in turn imply a congruence of conformal geodesics. Observe that, the coordinate $t$ is not well defined at the cosmological and black hole horizons, located at $r=r_{c}$ and $r=r_{b}$, respectively. Therefore, to describe a conformal geodesic crossing the horizons it is necessary to replace $t$ by either $u$ or $v$; the retarded and advanced null coordinates defined in equation (6). The $\tilde{\boldsymbol{g}}$-normalisation condition in the $(u, r)$ and $(v, r)$ coordinates is written as

$$
D(r) u^{\prime 2}-2 u^{\prime} r^{\prime}=1, \quad D(r) v^{2}+2 v^{\prime} r^{\prime}=1
$$

respectively. The normalisation condition (44) renders

$$
u^{\prime}=\frac{r^{\prime} \pm \sqrt{D(r)+r^{\prime 2}}}{D(r)}, \quad v^{\prime}=\frac{-r^{\prime} \pm \sqrt{D(r)+r^{\prime 2}}}{D(r)} .
$$

Using the chain rule and equations 45 and (41) one gets

$$
\begin{aligned}
& \frac{\mathrm{d} u}{\mathrm{~d} r}=\frac{1}{D(r)}\left(1 \pm \frac{|\gamma+\beta r|}{\sqrt{(\gamma+\beta r)^{2}-D(r)}}\right) \\
& \frac{\mathrm{d} v}{\mathrm{~d} r}=\frac{1}{D(r)}\left(-1 \pm \frac{|\gamma+\beta r|}{\sqrt{(\gamma+\beta r)^{2}-D(r)}}\right)
\end{aligned}
$$

Remark. For the subsequent analysis observe that analysing expression 46a for $u$ choosing the second sign is equivalent to analyse $-v$ choosing the first sign in $46 \mathrm{~b}$ and vice versa.

In the following Sections we carry out a case-by-case discussion of the solutions of the reduced conformal geodesics of Section 3.6 for the various spherically symmetric vacuum spacetimes with Cosmological constant introduced in Section 2 . 


\subsection{Explicit expressions for the reduced conformal deviation equations}

A direct calculation shows that if the Lorentzian metric $\tilde{\boldsymbol{l}}$ has the form of equation (36) then

$$
R[\tilde{\boldsymbol{l}}]=-\partial_{r}^{2} D(r)
$$

Therefore, equation (33) takes the form

$$
\not D_{\tilde{\boldsymbol{x}}^{\prime}} \not \tilde{\boldsymbol{x}}^{\prime} \tilde{\omega}=\left(\beta^{2}-\frac{1}{2} \partial_{r}^{2} D(r)\right) \tilde{\omega}+\not D_{\tilde{z}} \beta
$$

In order to derive initial data for $\tilde{\omega}$ it is necessary to specify the initial data for the deviation vector $\boldsymbol{z}$. To do so, first observe that we can parametrise the congruence as $r=r\left(\tilde{\tau}, r_{\star}\right)$ where $r_{\star}$ determines the intersection of the curve with the initial hypersurface $\mathcal{S}$. Alternatively, considering an approach similar to the one used in [14] we can make use of the standard isotropic coordinate defined by the relations in (5) and use $\rho_{\star}$ to parametrise the congruence. This approach leads to the choice $\boldsymbol{z}_{\star}=\boldsymbol{\partial}_{\rho_{\star}}$ for the initial data for the deviation vector. Accordingly, we consider equation (47) with the choice $\tilde{\boldsymbol{z}}_{\star}=\boldsymbol{\partial}_{\rho_{\star}}$. Consistent with this choice equation (47) reads

$$
\frac{\mathrm{d}^{2} \tilde{\omega}}{\mathrm{d} \tilde{\tau}^{2}}=\left(\beta^{2}-\frac{1}{2} \partial_{r}^{2} D(r)\right) \tilde{\omega}+\frac{\partial \beta}{\partial \rho_{\star}}, \quad \frac{\partial \beta}{\partial \rho_{\star}}=\frac{r_{\star} \sqrt{D_{\star}}}{\rho_{\star}} \frac{\partial \beta}{\partial r_{\star}},
$$

where $r=r\left(\tilde{\tau}, r_{\star}\right)$. The solution of this equation will be determined once the initial conditions $\tilde{\omega}_{\star} \equiv \tilde{\omega}\left(0, r_{\star}\right)$ and $\tilde{\omega}_{\star}^{\prime} \equiv \tilde{\omega}^{\prime}\left(0, r_{\star}\right)$ have been prescribed and the corresponding value of $\beta$ replaced. To compute the initial data for $\tilde{\omega}$ we use equation 32 with

$$
\boldsymbol{\epsilon}_{\tilde{\boldsymbol{l}}}=\mathbf{d} t \wedge \mathbf{d} r, \quad \tilde{\boldsymbol{x}}_{\star}^{\prime}=t_{\star}^{\prime} \partial_{t}+r_{\star}^{\prime} \boldsymbol{\partial}_{r}, \quad \tilde{\boldsymbol{z}}_{\star}=\boldsymbol{\partial}_{\rho_{\star}} .
$$

From the chain rule one finds that

$$
\partial_{\rho_{\star}}=\frac{r_{\star}}{\rho_{\star}} \sqrt{D_{\star}} \partial_{r_{\star}}
$$

Hence

$$
\begin{aligned}
\tilde{\omega}_{\star} & =(\mathbf{d} t \wedge \mathbf{d} r)\left(t^{\prime} \boldsymbol{\partial}_{t}+r^{\prime} \boldsymbol{\partial}_{r}, \frac{r_{\star}}{\rho_{\star}} \sqrt{D_{\star}}\left(\frac{\partial r}{\partial r_{\star}} \boldsymbol{\partial}_{r}+\frac{\partial t}{\partial r_{\star}} \boldsymbol{\partial}_{t}\right)\right) \\
& =\frac{r_{\star}}{\rho_{\star}} \sqrt{D_{\star}}\left(t^{\prime} \frac{\partial r}{\partial r_{\star}}+r^{\prime} \frac{\partial t}{\partial r_{\star}}\right) .
\end{aligned}
$$

Combining expression 49 with the initial data (51) we get the initial conditions

$$
\tilde{\omega}_{\star}=\frac{r_{\star}}{\rho_{\star}}, \quad \tilde{\omega}_{\star}^{\prime}=0 .
$$

\section{Analysis of the conformal geodesics in the subextremal Schwarzschild-de Sitter case}

In accordance with the discussion of Section 2.1. for the subextremal Schwarzschild-de Sitter spacetime it is assumed that $\lambda>0$ and $0<M<2 /(3 \sqrt{3})$. In this case one expects to be able to construct a congruence of conformal geodesics which combines characteristics of analogous congruences in the de Sitter and Schwarzschild spacetime.

\subsection{Basic setup}

The de Sitter spacetime can be covered by a congruence of conformal geodesics which have no initial acceleration - see 13, 17. Based on this result we consider as initial hypersurface the time symmetric slice $\tilde{\mathcal{S}}=\{t=0\}$ and require the conformal geodesics to be orthogonal to $\tilde{\mathcal{S}}$ - see 
Figure 5. Exploiting the periodicity of the spacetime, it is sufficient to restrict the analysis to initial data in the range

$$
r_{b} \leq r_{\star} \leq r_{c} .
$$

If $r_{\star} \neq r_{b}, r_{c}$ then we consider initial data for the congruence of conformal geodesics of the form

$$
t_{\star}=0, \quad t_{\star}^{\prime}=\frac{1}{\sqrt{D_{\star}}}, \quad r_{\star}^{\prime}=0, \quad \tilde{\beta}_{t \star}=0, \quad \tilde{\beta}_{r \star}=0,
$$

so that the tangent vector $\tilde{\boldsymbol{x}}^{\prime}$ coincides with the future unit normal to $\tilde{\mathcal{S}}$. It follows that in this case $\beta^{2}=0$ so that the resulting congruence of conformal geodesics is, after reparametrisation, a congruence of metric geodesics. Metric geodesics in the Schwarzschild-de Sitter spacetime have been studied in the literature - see [10]. For completeness, we here we carry out an independent analysis adapted to the present setting. Observe that equation (41) together with the condition $\beta^{2}=0$ readily yields

$$
\gamma=\sqrt{D_{\star}} .
$$

Using the latter one can write

$$
\gamma^{2}-D(r)=\frac{r-r_{\star}}{r}\left(r^{2}+r r_{\star}-\frac{M}{r_{\star}}\right) .
$$

For convenience of the subsequent discussion we introduce the polynomial

$$
\begin{aligned}
Q(r) & \equiv r^{2}+r r_{\star}-\frac{M}{r_{\star}}, \\
& =\left(r-\alpha_{-}\right)\left(r-\alpha_{+}\right),
\end{aligned}
$$

with

$$
\alpha_{ \pm}=r_{\star}\left(-\frac{1}{2} \pm \sqrt{\frac{1}{4}+\frac{M}{r_{\star}^{3}}}\right) .
$$

If $r_{\star}=r_{c}, r_{b}$ then the last three conditions in equation (51) can be still regarded a valid initial data but not the conditions involving $\tilde{t}_{\star}$. The cases $r_{\star}=r_{b}$ and $r_{\star}=r_{c}$ and will be discussed in Sections 4.2 .4 and 4.2.5. It is convenient to define

$$
r_{\circledast} \equiv\left(\frac{M}{2}\right)^{1 / 3} \text {. }
$$

The role of $r_{\circledast}$ will be clarified Section 4.2.1. An immediate observation regarding the location of $r_{\circledast}$ is the content of the following lemma.

Lemma 1. For $0<M<2 / 3 \sqrt{3}$ one has that

$$
r_{b}<r_{\circledast}<r_{c} .
$$

Proof. In the parametrisation introduced in equation 111 we have

$$
r_{\circledast}=\left(\frac{\cos \phi}{3 \sqrt{3}}\right)^{1 / 3} .
$$

In addition, if $\phi \in(0, \pi / 2)$ one has the inequalities

$$
\begin{array}{llll}
\left(\frac{\cos \phi}{3 \sqrt{3}}\right)^{1 / 3}>\frac{1}{\sqrt{3}} \cos \frac{\phi}{3}-\sin \frac{\phi}{3} & \text { so that } & r_{\circledast}>r_{b}, \\
\left(\frac{\cos \phi}{3 \sqrt{3}}\right)^{1 / 3}<\frac{1}{\sqrt{3}} \cos \frac{\phi}{3}+\sin \frac{\phi}{3} & \text { so that } & r_{\circledast}<r_{c} .
\end{array}
$$


Remark 3. Observe that in the extremal case one has $M=2 / 3 \sqrt{3}$ consequently $r_{\circledast}=r_{\mathcal{H}}$ where $r_{\mathcal{H}}$ denotes the location of the Killing horizon.

Given that $\boldsymbol{x}_{\star}^{\prime}$ has been chosen to be orthogonal to $\tilde{\mathcal{S}}$ and the range of $r_{\star}$ is bounded, we can set

$$
\tilde{\boldsymbol{\beta}}_{\star}=\boldsymbol{\beta}_{\star}=0, \quad \Theta_{\star}=1, \quad \dot{\Theta}_{\star}=0 .
$$

To simplify the subsequent expressions recall from Section 2 that one can set $\lambda=-3 \epsilon$ with $\epsilon=-1$ in the case of a de-Sitter like Cosmological constant. Consistent with this choice, equations (19)(20) render

$$
\Theta=1-\frac{\tau^{2}}{4}
$$

From this expression one can determine the relation between $\tilde{\tau}$ and $\tau$ using equation (21). A calculation gives

$$
\tilde{\tau}=2 \operatorname{arctanh}\left(\frac{\tau}{2}\right) .
$$

Observe that

$$
\lim _{\tau \rightarrow 2} \tilde{\tau}(\tau)=\infty,
$$

so that the conformal boundary is reached in a finite value of the unphysical proper time $\tau$.

Remark 4. The coordinate $t$ is not well-defined at the points where $D(r)$ vanishes. In the subextremal case, this corresponds to the Cosmological and black hole horizons while in the extremal case this corresponds to the Killing horizon.

To analyse the geodesics crossing the horizon one has to parametrise the curve in terms of the coordinates $(u, r)$ or $(v, r)$, where $u$ and $v$ are advanced and retarded null coordinates. Setting $\beta=0$ equations (45) and 41) render

$$
u^{\prime}=\frac{1}{D(r)}\left(\sqrt{\gamma^{2}-D(r)} \pm|\gamma|\right), \quad v^{\prime}=\frac{1}{D(r)}\left(-\sqrt{\gamma^{2}-D(r)} \pm|\gamma|\right) .
$$

Choosing the second sign in equation (56) and using L'Hôpital rule one finds

$$
\lim _{r \rightarrow r_{c}} u^{\prime}=-\frac{1}{2|\gamma|},
$$

Similarly, choosing the first sign in equation 56 one gets

$$
\lim _{r \rightarrow r_{b}} v^{\prime}=\frac{1}{2|\gamma|} .
$$

Therefore, one can use the coordinates $(u, r)$ to describe the curves crossing the Cosmological horizon at $r=r_{c}$. Similarly, the coordinates $(v, r)$ can be used to analyse curves crossing the black hole horizon at $r=r_{b}$.

\subsection{Qualitative analysis of the behaviour of the curves}

In this Section we analyse the different qualitative behaviours of the conformal geodesics defined by the initial conditions introduced in the previous Section. As it will be seen, in broad terms, there are three types of conformal geodesics: those that escape to the asymptotic points, those that escape to the conformal boundary and those falling into the singularity.

\subsubsection{Conformal geodesics with constant $r$ (critical curve).}

In this Section we discuss whether it is possible to have conformal geodesics for which $r$ is constant. Substituting the conditions $r^{\prime \prime}=r^{\prime}=0$ in equation 39 one readily obtains the condition

$$
\partial_{r} D(r)=\frac{M}{r^{2}}-2 r=0 .
$$




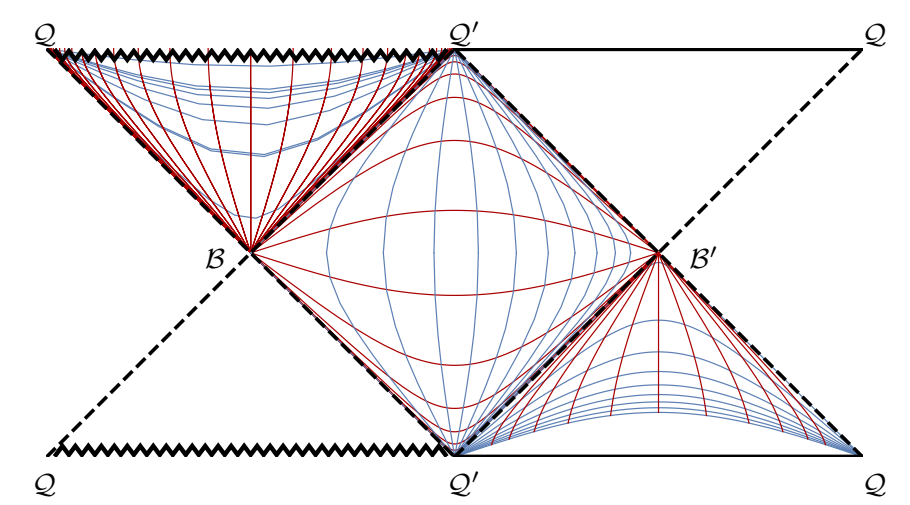

Figure 4: Curves with constant $t$ and $r$ (red and blue respectively) are plotted on the Penrose diagram of the subextremal Schwarzschild-de Sitter spacetime. Curves of constant $t$ intersect the bifurcation spheres $\mathcal{B}, \mathcal{B}^{\prime}$ while the curves of constant $r$ approach the asymptotic points $\mathcal{Q}$ and $\mathcal{Q}^{\prime}$.

The latter implies

$$
r=r_{\circledast} \equiv\left(\frac{M}{2}\right)^{1 / 3} .
$$

The curve $r(\tau)=r_{\star}=r_{\circledast}$ will be called the critical curve.

Remark 5. Notice that the curve $r=r_{\star}=r_{\circledast}$ never crosses the horizon since by virtue of Lemma 1 one has $r_{b}<r<r_{c}$. For this curves $r$ is always finite and they approach one of the asymptotic points $\mathcal{Q}$ or $\mathcal{Q}^{\prime}$.

In addition, one has the following result:

Lemma 2. Assume $\lambda>0$ and $0<M<2 /(3 \sqrt{3})$, then one has the chain of inequalities

$$
\begin{array}{lll}
\alpha_{-}<0<\alpha_{+}<r_{\circledast}<r_{\star} & \text { for } & r_{\star}>r_{\circledast}, \\
\alpha_{-}<0<r_{\star}<r_{\circledast}<\alpha_{+} & \text {for } & r_{\star}<r_{\circledast}, \\
\alpha_{-}<0<r_{\star}=r_{\circledast}=\alpha_{+} & \text {for } & r_{\star}=r_{\circledast} .
\end{array}
$$

Proof. To prove this result it is convenient to write $\alpha_{ \pm}$in terms of $r_{\circledast}$. One finds that

$$
\alpha_{ \pm}=\frac{r_{\star}}{2}\left(-1 \pm \sqrt{1+\left(\frac{2 r_{\circledast}}{r_{\star}}\right)^{3}}\right) .
$$

From the previous relation it is clear that $\alpha_{-}<0$ and that $\alpha_{-}<\alpha_{+}$. If $r_{\star}<r_{\circledast}$ then

$$
\sqrt{1+\left(\frac{2 r_{\circledast}}{r_{\star}}\right)^{3}}>3,
$$

which entails $\alpha_{+}>r_{\star}$. Also for any $z>2$ we have

$$
z\left(z^{2}-z-2\right)>0 \quad \text { if and only if } \quad \sqrt{1+z^{3}}>1+z .
$$

If we set $z=2 r_{\circledast} / r_{\star}$ in the last inequality and use the inequality 61 we deduce $\alpha_{+}>r_{\circledast}$. This proves the second chain of inequalities. Assume now that $r_{\star}>r_{\circledast}$. Then

$$
\sqrt{1+\left(\frac{2 r_{\circledast}}{r_{\star}}\right)^{3}}<3
$$

which entails $\alpha_{+}<r_{\star}$. If $z<2$ then

$$
z\left(z^{2}-z-2\right)<0 \quad \text { if and only if } \quad \sqrt{1+z^{3}}<1+z .
$$


Hence, setting $z=2 r_{\circledast} / r_{\star}$ in the last inequality and using, again, inequality (61) yields $\alpha_{+}<r_{\circledast}$ thus proving the first chain of inequalities. Finally, the last condition follows after setting $r_{\star}=r_{\circledast}$ in expression 60 .

Using equation 42 and the initial data one readily finds that along the critical curve

$$
t=t(\tilde{\tau})=\frac{\tilde{\tau}}{\sqrt{D_{\circledast}}} .
$$

Hence, $t \rightarrow \infty$ as $\tilde{\tau} \rightarrow \infty$. Alternatively, using formula (55), one can express $t$ in terms of the unphysical proper time. One obtains

$$
t=\frac{2}{\sqrt{D_{\circledast}}} \operatorname{arctanh}\left(\frac{\tau}{2}\right) .
$$

To complete the discussion observe that that along the critical curve one has $r=r_{\star}=r_{\circledast}$, so that along the critical curve $f_{\circledast}=f\left(r_{\circledast}\right)$ is a constant. Using equations (6) and (65) one concludes that along the critical curve

$$
u(\tau)=\frac{2}{\sqrt{D_{\circledast}}} \arctan \left(\frac{\tau}{2}\right)-r_{\circledast}, \quad v(\tau)=\frac{2}{\sqrt{D_{\circledast}}} \arctan \left(\frac{\tau}{2}\right)+r_{\circledast},
$$

thus at $\tau=2$ where $\Theta(\tau)$ vanishes, one has $u=\infty$ and $v=\infty$.

Asymptotic behaviour of the critical curve. In the rest of this Section we will analyse more closely the behaviour of the critical curve. Exploiting the above notation and using the initial data (51), the integral 43a) can be then rewritten as

$$
\tilde{\tau}=\int_{r_{\star}}^{r} \sqrt{\frac{\bar{r}}{\left(\bar{r}-r_{\star}\right)\left(\bar{r}-\alpha_{-}\left(r_{\star}\right)\right)\left(\bar{r}-\alpha_{+}\left(r_{\star}\right)\right)}} \mathrm{d} \bar{r} .
$$

To study the behaviour close to the critical curve consider $r_{\star}=(1+\epsilon) r_{\circledast}$. For small $\epsilon>0$ and $\bar{r}>r_{\star}$ one can expand the right hand side of equation (66) in Taylor series as

$$
\tilde{\tau}=\int_{r_{\star}}^{r} \sqrt{\frac{\bar{r}}{\bar{r}+2 r_{\circledast}}}\left(\frac{1}{\bar{r}-r_{\circledast}}-\frac{3 r_{\circledast}^{2} \bar{r} \epsilon^{2}}{2\left(\bar{r}-r_{\circledast}\right)^{3}}\right) \mathrm{d} \bar{r}+\mathcal{O}\left(\epsilon^{3}\right) .
$$

Integrating we obtain

$$
\begin{aligned}
\tilde{\tau}= & -\frac{2}{\sqrt{3}} \operatorname{arctanh}\left(\sqrt{3} \sqrt{\frac{1+\epsilon}{3+\epsilon}}\right)+2 \ln \left(\sqrt{r_{\circledast}(1+\epsilon)}+\sqrt{r_{\circledast}(3+\epsilon)}\right)-\frac{2}{\sqrt{3}} \operatorname{arctanh}\left(\frac{3 r}{r+2 r_{\circledast}}\right) \\
& +2 \ln \left(\sqrt{\tilde{r}}+\sqrt{r+2 r_{\circledast}}\right)-\frac{3}{4} r_{\circledast} \sqrt{1+2 r_{\circledast}}(1+2 \epsilon)-\frac{3}{4} r_{\circledast}^{2} \sqrt{1+2 r_{\circledast}} \frac{\left(2 r-r_{\circledast}\right) \epsilon^{2}}{\left(r_{\circledast}-r\right)^{2}}+\mathcal{O}\left(\epsilon^{3}\right) .
\end{aligned}
$$

As expected, the last expression diverges as $\epsilon \rightarrow 0$. The divergent term can be expanded for small $\epsilon>0$ as

$$
\operatorname{arctanh}\left(\sqrt{3} \sqrt{\frac{1+\epsilon}{3+\epsilon}}\right)=\frac{1}{2} \ln \left(\left|-\frac{6}{\epsilon}+4+\frac{\epsilon}{6}+\mathcal{O}\left(\epsilon^{2}\right)\right|\right)
$$

and the second term can be expanded as

$$
\ln \left(\sqrt{r_{\circledast}(1+\epsilon)}+\sqrt{r_{\circledast}(3+\epsilon)}\right)=\ln \left((1+\sqrt{3}) \sqrt{r_{\circledast}}\right)+\frac{\epsilon}{2 \sqrt{3}}-\frac{\epsilon^{2}}{6 \sqrt{3}}+\mathcal{O}\left(\epsilon^{3}\right) .
$$

Hence, to leading order one has

$$
\tilde{\tau}(r)=\frac{1}{\sqrt{3}} \ln \epsilon+f(r)+\mathcal{O}(\epsilon)
$$


where

$$
f(r)=2 \ln \left((1+\sqrt{3}) \sqrt{r_{\circledast}}\right)-\frac{2}{\sqrt{3}} \operatorname{arctanh}\left(\frac{3 r}{r+2 r_{\circledast}}\right)+2 \ln \left(\sqrt{r}+\sqrt{r+2 r_{\circledast}}\right)-\frac{3}{4} r_{\circledast} \sqrt{1+2 r_{\circledast}} .
$$

Rewriting the last expression in terms of the unphysical proper time using equation (55) one has, to leading order, that

$$
\tau(\epsilon)=\frac{2\left(\epsilon^{p}-1\right)}{\epsilon^{p}+1}
$$

where $p=1 / \sqrt{3}$. Differentiating we get

$$
\frac{\mathrm{d} \tau}{\mathrm{d} \epsilon}=\frac{4 p \epsilon^{p-1}}{\left(\epsilon^{p}+1\right)^{2}} .
$$

Since $p<1$ then one has that $\mathrm{d} \tau / \mathrm{d} \epsilon$ diverges as $\epsilon \rightarrow 0$. Consequently, the critical curve becomes tangent to the horizon as it approaches the asymptotic points $\mathcal{Q}$ and $\mathcal{Q}^{\prime}$. In other words, the critical curve becomes null asymptotically. This behaviour is similar to that observed in the Schwarzschild spacetime where $\mathcal{Q}$ plays the role of timelike infinity $i^{+}$as discussed in [5] and the subextremal Reissner-Nordström spacetime in [14].

Remark 6. The change in the the causal behaviour of the critical curve discussed in the previous Section is evidence of the strong singular behaviour of the conformal structure at the asymptotic points.

\subsubsection{Conformal geodesics with $r_{\circledast}<r_{\star} \leq r_{c}$}

Direct evaluation of equation (39) at $\tilde{\tau}=0$ for $r_{\star}>r_{\circledast}$ shows that $r_{\star}^{\prime \prime}>0$. As $r_{\star}^{\prime}=0$ one has that $r_{\star}$ is a local minimum of $r$. Hence, the curves with $r_{\circledast}<r_{\star} \leq r_{c}$ have $r$ initially increasing -i.e $r^{\prime}>0$. Accordingly, making use of the relations see equations (52) and(53) one is led to analyse the ordinary differential equation

$$
r^{\prime}=\sqrt{D_{\star}-D(r)}=\sqrt{\frac{r-r_{\star}}{r_{\star}} Q(r)} .
$$

Making use of Lemma2 2 one sees that the turning points of the equation are located at a value of $r$ which is smaller than $r_{\star}$. As $r$ is initially increasing it follows then that $r^{\prime} \neq 0$ for $\tilde{\tau} \in(0, \infty)$. Moreover, as $r>r_{\circledast}$ it follows that $r^{\prime \prime} \neq 0$ for $\tilde{\tau} \in(0, \infty)$. Finally, from equation 690 it follows that for the initial data under consideration $r^{\prime} \rightarrow \infty$ if and only if $r \rightarrow \infty$. It only remains to be checked that $r^{\prime}$ (or $r$ ) do not blow up in a finite amount of physical proper time $\tilde{\tau}$. From equation 69 the physical proper time is given by

$$
\tilde{\tau}=\int_{r_{\star}}^{r} \sqrt{\frac{r_{\star} \bar{r}}{\left(\bar{r}-r_{\star}\right)\left(r_{\star} \bar{r}\left(\bar{r}+r_{\star}\right)-M\right)}} \mathrm{d} \bar{r} .
$$

According to Lemma 2 if $r_{\star}>r_{\circledast}$ one has that $r_{\circledast}>\alpha_{+}$. Then, for any $r>r_{\circledast}$ it holds that $r>\alpha_{+}$. Given that $r_{\star}>0$ we have

$$
r_{\star} r\left(r+r_{\star}\right)-M>0 .
$$

Therefore the integral 700 is real for any $\tilde{r}>r_{\star}$. In addition, one has that

$$
\frac{r_{\star} r}{\left(r-r_{\star}\right)\left(r_{\star} r\left(r+r_{\star}\right)-M\right)} \geq \frac{r_{\star} r}{r_{\star} r\left(r-r_{\star}\right)\left(r+r_{\star}\right)}=\frac{1}{r^{2}-r_{\star}^{2}} \geq \frac{1}{r^{2}} .
$$

Thus

$$
\int_{r_{\star}}^{r} \sqrt{\frac{r_{\star} \bar{r}}{\left(\bar{r}-r_{\star}\right)\left(r_{\star} \bar{r}\left(\bar{r}+r_{\star}\right)-M\right)}} \mathrm{d} \bar{r} \geq \int_{r_{\star}}^{r} \sqrt{\frac{1}{\bar{r}^{2}}} \mathrm{~d} \bar{r}=\log r-\log r_{\star} .
$$

Hence

$$
r(\tilde{\tau}) \leq r_{\star} e^{\tilde{\tau}},
$$

showing that $r$ does not blow up in finite physical proper time. 
Remark 7. Summarising, the analysis in the previous paragraphs shows that if $r_{\circledast}<r_{\star} \leq r_{c}$, then $r(\tilde{\tau}) \rightarrow \infty$ as $\tilde{\tau} \rightarrow \infty$.

As discussed in a remark given in Section 4.1 the coordinate $t$ is not well behaved at the horizon. To follow the curves crossing the Cosmological horizon at $r=r_{c}$ one needs to use null coordinates $(u, r)$. In the following we first show that along the conformal geodesics the coordinate $u$ remains well defined for any finite value of $r$, in particular, for $r=r_{c}$. Then, we show that $u$ is finite at the conformal boundary. As discussed in Section 4.1 it is enough to analyse equation (46a) choosing the second sign so that $\lim _{r \rightarrow r_{b}} u^{\prime}=-1 / 2|\gamma|$. To start the discussion observe that the coordinate $u$ is determined by integration of equation $46 \mathrm{a}$ by

$$
u(r)-u_{\star}=\int_{r_{\star}}^{r} \frac{1}{D(\bar{r})}\left(1-\frac{|\gamma|}{\sqrt{\gamma^{2}-D(\bar{r})}}\right) \mathrm{d} \bar{r} .
$$

Analysis for the region with $r_{\star} \leq r_{c}$. Observe that, by continuity, one has that $\left|u(r)-u_{\star}\right|$ for $r \in\left[r_{\star}, r_{c}\right]$ would diverge if and only if the derivative $\mathrm{d} u / \mathrm{d} r$ diverges for some $r_{q} \in\left[r_{\star}, r_{c}\right]$. Direct inspection of equation (46a) using expressions (8), (9) and (53) show that $\mathrm{d} u / \mathrm{d} r$ is finite for $r \in\left[r_{\star}, r_{c}\right)$. Thus it only remains to compute the limit

$$
\lim _{r \rightarrow r_{c}} \frac{\mathrm{d} u}{\mathrm{~d} r} .
$$

Choosing the second sign in equation 46a and rearranging one has

$$
\frac{\mathrm{d} u}{\mathrm{~d} r}=\frac{1}{D(r)}\left(\frac{\sqrt{\gamma^{2}-D(r)}-|\gamma|}{\sqrt{\gamma^{2}-D(r)}}\right)
$$

A direct application of the L'Hôpital rule shows that

$$
\lim _{r \rightarrow r_{c}} \frac{\mathrm{d} u}{\mathrm{~d} r}=-\frac{1}{2 \gamma^{2}} .
$$

Consequently $u(r)$ does not diverge for $r \in\left[r_{\star}, r_{c}\right]$. In other words

$$
\left|u\left(r_{c}\right)-u_{\star}\right| \leq k^{2}
$$

for some constant $k$.

Analysis for the region with $r>r_{c}$. First let us found an appropriate estimate for the integral (71) for $r>r_{c}$ and show that the coordinate $u$ acquires a finite value at $\mathscr{I}$. Using that $D(r) \leq 0$ for $r>r_{c}$, we observe that

$$
\sqrt{\gamma^{2}-D(r)} \geq|\gamma|
$$

Recall that according to the initial data given in Section 4.1 one has $\gamma=\sqrt{D}_{\star} \neq 0$. Exploiting the last observation we find an estimate for $|u|$ as follows:

$$
\begin{aligned}
\left|u(r)-u\left(r_{c}\right)\right|= & \left|\int_{r_{c}}^{r} \frac{1}{D(\bar{r})}\left(1-\frac{|\gamma|}{\sqrt{\gamma^{2}-D(\bar{r})}}\right) \mathrm{d} \bar{r}\right| \\
& \leq \int_{r_{c}}^{r} \frac{1}{|D(\bar{r})|}\left|1-\frac{|\gamma|}{\sqrt{\gamma^{2}-D(\bar{r})}}\right| \mathrm{d} \bar{r} .
\end{aligned}
$$

To estimate the last integral of expression (75) observe that inequality (74) can be written as $1 \geq|\gamma| / \sqrt{\gamma^{2}-D(r)}$, equivalently

$$
1-\frac{|\gamma|}{\sqrt{\gamma^{2}-D(r)}} \geq 0
$$


thus

$$
\frac{1}{|D(r)|}\left|1-\frac{|\gamma|}{\sqrt{\gamma^{2}-D(r)}}\right|=\frac{1}{|D(r)|}\left(1-\frac{|\gamma|}{\sqrt{\gamma^{2}-D(r)}}\right) .
$$

Using again inequality (74), written as $1 \leq \sqrt{\gamma^{2}-D(r)} /|\gamma|$ and taking into account that $D(r) \leq$ 0 , we get the following

$$
\begin{gathered}
\frac{1}{|D(r)|}\left(1-\frac{|\gamma|}{\sqrt{\gamma^{2}-D(r)}}\right) \leq \\
\frac{1}{|D(r)|}\left(\frac{\sqrt{\gamma^{2}-D(r)}}{|\gamma|}-\frac{|\gamma|}{\sqrt{\gamma^{2}-D(r)}}\right)=\frac{1}{|D(r)|} \frac{\gamma^{2}-D(r)-\gamma^{2}}{|\gamma| \sqrt{\gamma^{2}-D(r)}} \\
=\frac{1}{|D(r)|} \frac{|D(r)|}{|\gamma| \sqrt{\gamma^{2}-D(r)}}=\frac{1}{|\gamma| \sqrt{\gamma^{2}-D(r)}} .
\end{gathered}
$$

Finally, using again inequality 74 , we have that

$$
\frac{1}{\sqrt{\gamma^{2}-D(r)}} \leq \frac{1}{|\gamma|}
$$

thus,

$$
\frac{1}{|\gamma| \sqrt{\gamma^{2}-D(r)}} \leq \frac{1}{\gamma^{2}}
$$

Following the last chain of inequalities one can estimate the integral 75 as follows

$$
\left|u(r)-u\left(r_{c}\right)\right|=\left|\int_{r_{\star}}^{r} \frac{1}{D(\bar{r})}\left(1-\frac{|\gamma|}{\sqrt{\gamma^{2}-D(\bar{r})}}\right) \mathrm{d} \bar{r}\right| \leq \int_{r_{\star}}^{r} \frac{1}{\gamma^{2}} \mathrm{~d} \bar{r}=\frac{r-r_{\star}}{\gamma^{2}} .
$$

Using equation $10 \mathrm{c}$ and the triangle inequality one can verify that the location of the cosmological horizon is constrained by

$$
1-\frac{1}{\sqrt{3}} \leq r_{c} \leq 1+\frac{1}{\sqrt{3}}
$$

Now, consider

$$
r_{\bullet}>\max \left\{1, r_{c}\right\}
$$

and let $u_{\infty} \equiv \lim _{r \rightarrow \infty} u$. Using this notation we can estimate $\left|u_{\infty}-u\left(r_{\bullet}\right)\right|$ as follows

$$
\begin{aligned}
\left|u_{\infty}-u\left(r_{\bullet}\right)\right|= & \left|\int_{r_{\bullet}}^{\infty} \frac{1}{D(\bar{r})}\left(1-\frac{|\gamma|}{\sqrt{\gamma^{2}-D(\bar{r})}}\right) \mathrm{d} \bar{r}\right| \leq \int_{r_{\bullet}}^{\infty}\left|\frac{1}{D(\bar{r})}\left(1-\frac{|\gamma|}{\sqrt{\gamma^{2}-D(\bar{r})}}\right)\right| \mathrm{d} \bar{r} \\
& \leq \int_{r_{\bullet}}^{\infty} \frac{1}{|D(\bar{r})|}+\frac{1}{|D(\bar{r})|}\left|\frac{|\gamma|}{\sqrt{\gamma^{2}-D(\bar{r})}}\right| \mathrm{d} \bar{r} \leq \int_{r_{\bullet}}^{\infty} \frac{2}{|D(\bar{r})|} \mathrm{d} \bar{r}
\end{aligned}
$$

In the last inequality we have used equation (74), in the form $|\gamma| / \sqrt{\gamma^{2}-D(r)} \leq 1$. Using the functional form for $D(r)$ in the subextremal case, one has that, for $r>r$.

$$
|D(r)|=-D(r)=\frac{1}{r}\left(r^{3}-r+m\right)>r^{2}-1 .
$$

Consequently,

$$
\frac{1}{|D(r)|}<\frac{1}{r^{2}-1} .
$$

Integrating the last inequality we get

$$
\int_{r_{\bullet}}^{r} \frac{2}{|D(\bar{r})|} \mathrm{d} \bar{r}<\ln \left|\frac{1-r}{1+r}\right|_{r_{\bullet}}^{r} .
$$


Thus,

$$
\left|u_{\infty}-u\left(r_{\bullet}\right)\right|<\ln \left|\frac{1+r_{\bullet}}{1-r_{\bullet}}\right| .
$$

Consequently, using expressions $(76,, 78)$ and the triangle inequality one obtains

$$
\left|u_{\infty}-u\left(r_{c}\right)\right| \leq\left|u_{\infty}-u\left(r_{\bullet}\right)\right|+\left|u\left(r_{\bullet}\right)-u\left(r_{c}\right)\right|<\ln \left|\frac{1+r_{\bullet}}{1-r_{\bullet}}\right|+\frac{r_{\bullet}-r_{c}}{\gamma^{2}}<\infty .
$$

Finally from expressions 73 and 79 one concludes that

$$
\left|u_{\infty}-u_{\star}\right| \leq\left|u_{\infty}-u\left(r_{c}\right)\right|+\left|u\left(r_{c}\right)-u\left(r_{\star}\right)\right|<\ln \left|\frac{1+r_{\bullet}}{1-r_{\bullet}}\right|+\frac{r_{\bullet}-r_{c}}{\gamma^{2}}+k^{2}<\infty .
$$

Remark 8. Summarising, the analysis in the previous paragraphs shows that the conformal geodesics with $r_{\circledast}<r_{\star} \leq r_{c}$ cross the Cosmological horizon at $r=r_{c}$ and eventually escape to the conformal boundary. In particular, the curves do not touch the asymptotic points $\mathcal{Q}$ and $\mathcal{Q}^{\prime}$ for which $u=\infty$ and $u=-\infty$.

\subsubsection{Conformal geodesics with $r_{b} \leq r_{*}<r_{\circledast}$}

Direct evaluation of equation (39) at $\tilde{\tau}=0$ for $r_{b} \leq r_{\star}<r_{\circledast}$ shows that $r_{\star}^{\prime \prime}<0$ so that $r_{\star}$ is a local maximum of $r$-that is, $r_{\star}^{\prime}<0$. Hence, the curves with $r_{b} \leq r_{\star}<r_{\circledast}$ have $r$ initially decreasing and one has

$$
r^{\prime}=-\sqrt{\gamma^{2}-D(r)}
$$

From the above relation we deduce that the proper time $\tilde{\tau}$ of the curves is given by

$$
\tilde{\tau}=\int_{r}^{r_{\star}} \sqrt{\frac{r_{\star} \bar{r}}{\left(r_{\star}-\bar{r}\right)\left(M-r_{\star} \bar{r}\left(\bar{r}+r_{\star}\right)\right)}} \mathrm{d} \bar{r} .
$$

Lemma 2 tells us that if $r_{\star}<r_{\circledast}$ one has that $\alpha_{-}<0<r_{\circledast}<\alpha_{+}$and therefore for any $0<r<r_{\circledast}$ it holds that $\alpha_{-}<r<\alpha_{+}$. Hence $Q(r)<0$ implies that

$$
M-r_{\star} r\left(r+r_{\star}\right)>0, \quad 0<r<r_{\circledast} .
$$

This means that the integrand in equation (81) is real for any $r$ in $\left[0, r_{\circledast}\right]$. In addition, one has that

$$
\frac{r_{\star} r}{\left(r_{\star}-r\right)\left(M-r_{\star} r\left(r+r_{\star}\right)\right)} \leq \frac{r_{\star}^{2}}{\left(r_{\star}-r\right)}, \quad 0<r<r_{\circledast} .
$$

Consequently, one can conclude that

$$
\tilde{\tau}=\int_{r}^{r_{\star}} \sqrt{\frac{r_{\star} \bar{r}}{\left(r_{\star}-\bar{r}\right)\left(M-r_{\star} \bar{r}\left(\bar{r}+r_{\star}\right)\right)}} \mathrm{d} \bar{r} \leq r_{\star}^{2} \int_{r}^{r_{\star}} \frac{\mathrm{d} \bar{r}}{\sqrt{r_{\star}-\bar{r}}}=2 r_{\star}^{2} \sqrt{r_{\star}-r} .
$$

This means that $\tilde{\tau}$ remains finite for any value of $r$ in the interval $\left[0, r_{\star}\right]$. In particular the geodesics reach the singularity $r=0$ in a finite value of the proper time $\tilde{\tau}$.

Behaviour across the black hole horizon at $r=r_{b}$. To complete the discussion of this class of conformal geodesics we now show that the advanced time coordinate $v$ can be used to follow the curves across the horizon at $r=r_{b}$ and that the value of $v$ remains finite as $r \rightarrow 0$. Given that $v^{\prime}$ is finite at $r=r_{b}$-cf. the limit in equation (58) - it follows that the coordinate $v$ has a finite value at $r=r_{b}$. Moreover, it can be readily verified that

$$
\lim _{r \rightarrow 0} v^{\prime}=0 \text {. }
$$

Thus, the coordinate $v$ acquires a finite limit $v_{\xi}$ as $r \rightarrow 0$.

Remark 9. Summarising, the conformal geodesics with $r_{b} \leq r_{*}<r_{\circledast}$ reach the black hole horizon at $r=r_{b}$ in a finite amount of proper time and fall into the singularity at $r=0$ also in a finite proper time. Further, the curves remain away from the asymptotic points $\mathcal{Q}$ and $\mathcal{Q}^{\prime}$ for which $v=\infty$ and $v=-\infty$. 


\subsubsection{Conformal geodesics through the bifurcation sphere of the cosmological hori-} zon

As shown in previous Sections the conformal geodesics with initial data $r_{\star}>r_{\circledast}$ arrive at the conformal boundary at a finite value of the unphysical proper time $\tau$. However it is possible that the conformal geodesics accumulate in certain region of $\mathscr{I}$. In order to see that this is not the case we analyse the behaviour of the conformal geodesics starting at the bifurcation sphere of the horizon at $r=r_{c}$.

In order to proceed with the analysis, it is convenient to introduce Kruskal type coordinates. First, recall that the Schwarzschild-de Sitter metric can be written as

$$
\tilde{\boldsymbol{g}}=\tilde{\boldsymbol{l}}-r^{2} \boldsymbol{\sigma},
$$

where the induced metric $\tilde{\boldsymbol{l}}$ on the quotient manifold $\tilde{\mathcal{M}} / S O(3)$ as defined in equation 36 . Introducing Kruskal coordinates via

$$
U=\frac{1}{2} \exp (b u), \quad V=\frac{1}{2} \exp (b v)
$$

where $u$ and $v$ are the Eddington-Finkelstein null coordinates as defined in equation (6) and $b$ is a constant which can be freely chosen. In this coordinate system the metric $\tilde{\boldsymbol{l}}$ reads

$$
\tilde{l}=G(r)(\mathbf{d} U \otimes \mathbf{d} V+\mathbf{d} V \otimes \mathbf{d} U),
$$

with

$$
G(r) \equiv \frac{D(r)}{b^{2}} \exp (-2 b r(r)),
$$

where $f(r)$ is the tortoise coordinate as defined in equation (7). A straightforward computation using (8) taking into account that $r_{-}<0<r_{b}<r_{c}$ renders

$$
\begin{array}{r}
r(r)=\frac{\left|r_{-}\right|}{\left(r_{c}+\left|r_{-}\right|\right)\left(r_{b}+\left|r_{-}\right|\right)} \ln \left(r+\left|r_{-}\right|\right)+\frac{r_{b}}{\left(r_{b}+\left|r_{-}\right|\right)\left(r_{c}-r_{b}\right)} \ln \left|r-r_{b}\right| \\
-\frac{r_{c}}{\left(r_{c}+\left|r_{-}\right|\right)\left(r_{c}-r_{b}\right)} \ln \left|r-r_{c}\right| .
\end{array}
$$

One can verify that $\lim _{r \rightarrow \infty} f(r)=0$ and that

$$
G(r)=\frac{1}{b^{2} r}\left(r+\left|r_{-}\right|\right)^{A_{n}}\left(r-r_{b}\right)^{A_{b}}\left(r-r_{c}\right)^{A_{c}}
$$

where $A_{n}, A_{b}, A_{c}$ are constants defined via

$$
A_{n} \equiv 1-\frac{2 b\left|r_{-}\right|}{\left(r_{c}+\left|r_{-}\right|\right)\left(r_{b}+\left|r_{-}\right|\right)}, \quad A_{b} \equiv 1-\frac{2 b r_{b}}{\left(r_{c}-r_{b}\right)\left(r_{b}+\left|r_{-}\right|\right)}, \quad A_{c} \equiv 1+\frac{2 b r_{c}}{\left(r_{c}-r_{b}\right)\left(r_{c}+\left|r_{-}\right|\right)}
$$

As discussed in [1] one cannot construct coordinates that are regular in the neighbourhood of the Cosmological and black hole horizons simultaneously. Since in this Section we are only interested in analysing the conformal geodesic starting at $r_{\star}=r_{c}$ we observe that setting

$$
b \equiv-\frac{1}{2 r_{c}}\left(r_{c}-r_{b}\right)\left(r_{c}+\left|r_{-}\right|\right)
$$

so that $A_{c}=0$, one gets

$$
\tilde{\boldsymbol{l}}=\frac{1}{b^{2} r}\left(r+\left|r_{-}\right|\right)^{A_{n}}\left(r-r_{b}\right)^{A_{b}}(\mathbf{d} U \otimes \mathbf{d} V+\mathbf{d} V \otimes \mathbf{d} U) .
$$

Introducing a further change of coordinates $T=U+V$ and $\Psi=V-U$ one obtains

$$
\tilde{\boldsymbol{l}}=\frac{1}{2 b^{2} r}\left(r+\left|r_{-}\right|\right)^{A_{n}}\left(r-r_{b}\right)^{A_{b}}(\mathbf{d} T \otimes \mathbf{d} T-\mathbf{d} \Psi \otimes \mathbf{d} \Psi),
$$


where the coordinates $T$ and $\Psi$ are related to $r$ and $t$ via

$$
T(r, t)=\cosh t \exp (b r(r)), \quad \Psi(r, t)=\sinh t \exp (b r(r)) .
$$

A straightforward computation renders the additional relation

$$
T^{2}-\Psi^{2}=\left(r+\left|r_{-}\right|\right)^{k_{n}}\left(r-r_{b}\right)^{k_{b}}\left(r-r_{c}\right),
$$

with $k_{n} \equiv 1-A_{n}<0$ and $k_{b} \equiv 1-A_{b}<0$. Observe that the metric (84) is regular at $r_{c}$. Moreover, notice that, since

$$
\lim _{r \rightarrow r_{c}} f(r)=\infty
$$

and $b<0$ then

$$
\lim _{r \rightarrow r_{c}} T=0, \quad \lim _{r \rightarrow r_{c}} \Psi=0 .
$$

Consequently, the conformal geodesic starting at the bifurcation sphere in these coordinates correspond to the geodesic with initial position $\left(T_{\star}, \Psi_{\star}\right)=(0,0)$. Consistent with the discussion of Sections 3.5 4.1 we consider curves with no evolution in the angular coordinates and $\beta=0$, so in these coordinates, the geodesic equations read

$$
\begin{aligned}
& T^{\prime \prime}+\frac{\partial \ln G}{\partial \Psi} T^{\prime} \Psi^{\prime}+\frac{1}{2} \frac{\partial \ln G}{\partial T}\left(T^{\prime 2}+\Psi^{\prime 2}\right)=0 \\
& \Psi^{\prime \prime}+\frac{\partial \ln G}{\partial T} T^{\prime} \Psi^{\prime}+\frac{3}{2} \frac{\partial \ln G}{\partial \Psi} \Psi^{\prime 2}-\frac{1}{2} \frac{\partial \ln G}{\partial \Psi} T^{\prime 2}=0
\end{aligned}
$$

where as before' denotes a derivative respect to the physical proper time $\tilde{\tau}$. Now, consider the curve with initial conditions

$$
T_{\star}=0, \quad \Psi_{\star}=0, \quad T_{\star}^{\prime} \neq 0, \quad \Psi_{\star}^{\prime}=0,
$$

and recall that in the asymptotic region the curves with constant $t$ approach the bifurcation sphere - see Figure 4. Moreover, observe that in the $(T, \Psi)$-coordinate system the curve with $t=0$ is described by $T=\exp (b r(r))$ and $\Psi=0$. In the subsequent discussion we show that this curve is the required geodesic with the initial data given in equation 89 . Assuming $\Psi=0$ equations 87 render the conditions

$$
\begin{aligned}
& T^{\prime \prime}+\frac{1}{2} \frac{\partial \ln G}{\partial T} T^{2}=0, \\
& \left.\frac{\partial \ln G}{\partial \Psi}\right|_{\Psi=0}=0 .
\end{aligned}
$$

Observe that equation can be rewritten as an equation for $r$ while equation (91) has to be verified with the implicit expression for $G(T, \Psi)$. Using the chain rule we have

$$
\left.\frac{\partial \ln G}{\partial \Psi}\right|_{\Psi=0}=\left.\left.\left(\frac{1}{G} \frac{\partial G}{\partial r}\right)\right|_{r=r_{c}} \frac{\partial r}{\partial \Psi}\right|_{\Psi=0} .
$$

Using the implicit function theorem and equation 86 one can compute $\partial r / \partial \Psi$ to obtain

$$
\frac{\partial r}{\partial \Psi}=\frac{-2 \Psi}{\left(r+\left|r_{-}\right|\right)^{k_{n}}\left(r-r_{b}\right)^{k_{b}}\left(1+\frac{k_{c}\left(r-r_{c}\right)}{r-r_{b}}+\frac{k_{c}\left(r-r_{c}\right)}{r+\left|r_{-}\right|}\right)} .
$$

Notice that $G\left(r_{c}\right) \neq 0$ and $\partial G /\left.\partial r\right|_{r=r_{c}} \neq 0$ while $\partial r / \partial \Psi$ vanishes for $\Psi=0$ as long as $r \neq r_{b}$. Since $r(\tilde{\tau}) \geq r_{c}>r_{b}$ one concludes that equation (91) is satisfied. To verify that this curve corresponds also the geodesic with initial data (89) observe that using the chain rule and the definition of the tortoise coordinate one has

$$
\frac{\mathrm{d} T}{\mathrm{~d} \tilde{\tau}}=b T(r) \frac{\mathrm{d} r}{\mathrm{~d} r} \frac{\mathrm{d} r}{\mathrm{~d} \tilde{\tau}}=\frac{b T(r)}{D(r)} \frac{\mathrm{d} r}{\mathrm{~d} \tilde{\tau}} .
$$


Since at $\tilde{\tau}=0$ one has $r=r_{\star}=r_{c}$ then

$$
T_{\star}^{\prime}=\lim _{r \rightarrow r_{c}} \frac{b T(r)}{D(r)} \frac{\mathrm{d} r}{\mathrm{~d} \tilde{\tau}} .
$$

To analyse this limit observe that

$$
T(r)=\exp (b r)=\sqrt{\left(r+\left|r_{-}\right|\right)^{k_{n}}\left(r-r_{b}\right)^{k_{b}}\left(r-r_{c}\right)} .
$$

Using equations 41 and with $\beta=0$ and $r_{\star}=r_{c}$ one has

$$
\frac{\mathrm{d} r}{\mathrm{~d} \tilde{\tau}}=\sqrt{\frac{\left(r-r_{c}\right)\left(r-\alpha_{-}\left(r_{c}\right)\right)\left(r-\alpha_{+}\left(r_{c}\right)\right)}{r}}
$$

where

$$
\alpha_{ \pm}\left(r_{c}\right)=\frac{r_{c}}{2}\left(-1 \pm \sqrt{1+\left(\frac{2 r_{\circledast}}{r_{c}}\right)^{3}}\right),
$$

substituting equation (8) one obtains

$$
\frac{T(r)}{D(r)} \frac{\mathrm{d} r}{\mathrm{~d} \tilde{\tau}}=-\sqrt{r\left(r-\alpha_{-}\left(r_{c}\right)\right)\left(r-\alpha_{+}\left(r_{c}\right)\right)\left(r+\left|r_{-}\right|\right)^{k_{n}-2}\left(r-r_{b}\right)^{k_{b}-2}}
$$

Evaluating the last expression at $r=r_{c}$ and using equation $(92)$ one concludes that $T_{\star}^{\prime} \neq 0$. Moreover one can verify that $T_{\star}^{\prime}$ is positive and finite. Finally, notice that

$$
\lim _{r \rightarrow \infty} T(r)=1 .
$$

Remark 10. The analysis in the previous paragraphs shows that conformal geodesics with $r_{\star}=r_{c}$ intersect the conformal boundary at $(T, \Psi)=(1,0)$. In terms of the retarded EddingtonFinkelstein null coordinate $u$ this corresponds to the condition $u=0$. Accordingly, these conformal geodesics bisect the Cosmological region of the spacetime, and in particular also the conformal boundary.

\subsubsection{Conformal geodesic through the bifurcation sphere of the black hole horizon}

The analysis of the previous Section can be adapted, mutatis mutandi, to the case of conformal geodesics starting at $r_{\star}=r_{b}$. In this case the function $b$ in the function $G(r)$ as given by equation (83) is set to

$$
b \equiv \frac{1}{2 r_{b}}\left(r_{c}-r_{b}\right)\left(r_{c}+\left|r_{-}\right|\right)
$$

so that

$$
\tilde{\boldsymbol{l}}=\frac{1}{2 b^{2} r}\left(r+\left|r_{-}\right|\right)^{A_{n}}\left(r-r_{c}\right)^{A_{c}}(\mathbf{d} T \otimes \mathbf{d} T-\mathbf{d} \Psi \otimes \mathbf{d} \Psi) .
$$

As $b>0$ and given that $\lim _{r \rightarrow r_{b}} x=-\infty$, it follows that

$$
\lim _{r \rightarrow r_{b}} T=\lim _{r \rightarrow r_{b}} \Psi=0 .
$$

Using the same methods employed in the analysis of the geodesics with $r_{\star}=r_{c}$, it can be readily shown that the curves with initial conditions given by

$$
T_{\star}=0, \quad \Psi_{\star}=0, \quad T_{\star}^{\prime} \neq 0, \quad \Psi_{\star}^{\prime}=0,
$$

and such that

$$
\Psi(\tilde{\tau})=0, \quad \tilde{\tau} \geq 0,
$$

are conformal geodesics. Moreover, it can be verified that

$$
\lim _{r \rightarrow 0} T=T_{\text {夕夕 }} \equiv \sqrt{\left|r_{-}\right|^{k_{n}} r_{b} r_{c}^{k_{c}}} \neq 0 .
$$

In what follows, we will denote by $\left(u_{\xi}, v_{\xi}\right)$ the value of the null coordinates $(u, v)$ associated to $(T, \Psi)=\left(T_{\text {夕夕 }}, 0\right)$. Notice that, in particular $u_{\text {多 }}=v_{\text {夕夕 }}$.

Remark 11. The analysis of the previous paragraphs shows that the black hole region is bisected by timelike conformal geodesics emanating from the bifurcation sphere at $r=r_{b}$. 


\subsection{Explicit expressions in terms of elliptic functions}

The integral of equation (43a) can be written in terms of special functions. In particular, using formulae 258.13 and 339.01 given in [2], with parameters

$$
a=r_{\star}, \quad b=\alpha_{+}, \quad c=0, \quad d=\alpha_{-},
$$

one can rewrite the integral (66) in terms of elliptic functions. Using these formulae one concludes that the substitution

$$
\operatorname{sn}^{2} \bar{w}=\left(\frac{\alpha_{+}-\alpha_{-}}{r_{\star}-\alpha_{-}}\right)\left(\frac{\bar{r}-r_{\star}}{\bar{r}-\alpha_{+}}\right),
$$

leads to

$$
\tilde{\tau}=\frac{2 r_{\star}}{\alpha^{2} \sqrt{r_{\star}\left(\alpha_{+}-\alpha_{-}\right)}}\left(\kappa^{2} w+\left(\alpha^{2}-\kappa^{2}\right) \Pi\left[\phi, \alpha^{2}, \kappa\right]\right),
$$

where sn denotes the Jacobian elliptic function, $\Pi\left[\phi, \alpha^{2}, \kappa\right]$ is the incomplete elliptic integral of the third kind and

$$
\begin{aligned}
\operatorname{sn}^{2} w \equiv\left(\frac{\alpha_{+}-\alpha_{-}}{r_{\star}-\alpha_{-}}\right)\left(\frac{r-r_{\star}}{r-\alpha_{+}}\right), & \alpha^{2} \equiv \frac{r_{\star}-\alpha_{-}}{\alpha_{+}-\alpha_{-}}, \\
\kappa^{2} \equiv \frac{\alpha_{+}\left(r_{\star}-\alpha_{-}\right)}{r_{\star}\left(\alpha_{+}-\alpha_{-}\right)}, & \phi \equiv \arcsin (\operatorname{sn} w) .
\end{aligned}
$$

In particular, it follows from the previous expressions and the general theory of elliptic functions that $\tilde{\tau}\left(r, r_{\star}\right)$ as defined by the right hand side of equation $(93)$, is an analytic function of its arguments - see e.g. [12. Notice that in Sections 4.1 4.2.3 we have already analysed the behaviour of $\tilde{\tau}\left(r, r_{\star}\right)$ by constructing suitable estimates. Using equation (55) one can write $\tau=2 \tanh \left(\tilde{\tau}\left(r, r_{\star}\right) / 2\right)$ and integrate $46 \mathrm{a}$ ) and $46 \mathrm{~b}$. From this discussion it follows that $u\left(\tau, r_{\star}\right)$ and $v\left(\tau, r_{\star}\right)$ are analytic functions of their arguments.

Remark 12. Observe that due to the periodicity in the spatial direction of the maximal extension of the spacetime - see Figure 1, to analyse the behaviour of a congruence of conformal geodesics considering initial data with $r_{b} \leq r_{\star} \leq r_{c}$ is sufficient.

\subsection{Analysis of the behaviour of the conformal deviation equations}

In Section 3.4 and 3.5 it was show how the requirement that the tangent vectors of the congruence $\dot{\boldsymbol{x}}$ and the deviation vector for the congruence $\boldsymbol{z}$ remain linearly independent can be studied by analysing a single scalar quantity $\tilde{\omega}$. The purpose of this Section is to discuss the evolution of $\tilde{\omega}$ to analyse the potential formation of conjugate points in the congruences of conformal geodesics constructed in the previous Sections.

To start the discussion observe that in the subextremal Schwarzschild-de Sitter case one has $\beta=0$, consequently, equation (48) takes the simpler form

$$
\frac{\mathrm{d}^{2} \tilde{\omega}}{\mathrm{d} \tilde{\tau}^{2}}=\left(1+\frac{M}{r^{3}}\right) \tilde{\omega}, \quad r \equiv r\left(\tilde{\tau}, r_{\star}\right) .
$$

We distinguish two possibilities according to whether $r_{\star}=r_{\circledast}$ or $r_{\star} \neq r_{\circledast}$.

\subsubsection{Conformal geodesics with $r_{\star}=r_{\circledast}$}

If $r_{\star}=r_{\circledast}$ then $r=r_{\circledast}$ as shown in Section 4.2.1. Hence, given that $r$ is a constant, the differential equation 94 with initial conditions given by equation 50 yields

$$
\tilde{\omega}(\tilde{\tau})=\frac{r_{\circledast}}{\rho_{\circledast}} \cosh \left(\tilde{\tau} \sqrt{1+\frac{M}{r_{\circledast}^{3}}}\right), \quad r_{\circledast}=r\left(\rho_{\circledast}\right) .
$$


Using equations (54)-(55) and (35) we obtain

$$
\tilde{\omega}(\tau)=r_{\circledast} \rho_{\circledast}\left(1-\frac{\tau^{2}}{4}\right) \cosh \left(\sqrt{1+\frac{M}{r_{\circledast}^{3}}} \operatorname{arctanh}\left(\frac{\tau}{2}\right)\right) .
$$

This function is clearly non-zero for any value of $\tau \in(-2,2)$ and thus no caustics develop along the curve defined by $r=r_{\circledast}$. We also deduce that

$$
\lim _{\tau \rightarrow \pm 2} \tilde{\omega}=\infty .
$$

\subsubsection{Conformal geodesics with $r_{\star} \neq r_{\circledast}$}

Observe that if $r_{\star} \neq r_{\circledast}$, then either $r_{\star}<r_{\circledast}$ and the conformal geodesics end at the singularity or $r_{\star}>r_{\circledast}$ and the conformal geodesics reach the conformal boundary $\mathscr{I}$. In any case one has that

$$
1+\frac{M}{r}>1
$$

The latter, together with equation 480 entails the differential inequality

$$
\frac{\mathrm{d}^{2} \omega}{\mathrm{d} \tilde{\tau}^{2}}>\omega
$$

It follows then that the scalars $\tilde{\omega}$ and $\omega \equiv \Theta \tilde{\omega}$ satisfy the inequalities

$$
\omega \geq \varpi, \quad \hat{\omega} \geq \Theta \varpi,
$$

where $\varpi$ is the solution of

$$
\frac{\mathrm{d}^{2} \varpi}{\mathrm{d} \tilde{\tau}^{2}}=\varpi, \quad \varpi\left(0, \rho_{\star}\right)=\frac{r_{\star}}{\rho_{\star}}, \quad \varpi^{\prime}\left(0, \rho_{\star}\right)=0 .
$$

The solution to this last differential equation is given by $\varpi=\left(r_{\star} / \rho_{\star}\right) \cosh \tilde{\tau}$. Using equations (54)-(55) we get the inequality

$$
\omega \geq\left(1-\frac{\tau^{2}}{4}\right) \frac{r_{\star}}{\rho_{\star}} \cosh \left(2 \operatorname{arctanh}\left(\frac{\tau}{2}\right)\right)=\frac{r_{\star}}{\rho_{\star}}\left(1+\frac{\tau^{2}}{4}\right)>0 .
$$

Consequently, one gets the limit

$$
\lim _{\tau \rightarrow \pm 2} \omega \geq \frac{2 r_{\star}}{\rho_{\star}}>0 .
$$

Hence, we conclude that the geodesics with $r_{\star}>r_{\circledast}$ which go to the conformal boundary $\mathscr{I}$ located at $\tau=2$ do not develop any caustic. Now, for the case $r_{\star}<r_{\circledast}$ in which the conformal geodesics fall into the singularity it is sufficient to observe that

$$
\tilde{\omega} \geq \frac{r_{\star}}{\rho_{\star}} \cosh (\tilde{\tau}) .
$$

Since the geodesics reach the singularity in a finite value of the physical proper time $\tilde{\tau}$ and $\cosh (\tilde{\tau}) \geq 2$ one conclude that the conformal geodesics falling into the singularity does not form caustics as well.

\subsection{Conformal Gaussian coordinates in the subextremal Schwarzschild- de Sitter spacetime}

In this Section we combine the results of the previous Sections to show how the congruence of conformal geodesics defined by the initial data in Section 4.1 covers the the whole maximal extension of the subextremal Schwarzschild-de Sitter spacetime. Accordingly, one can use this congruence to construct global conformal Gaussian coordinates. Because of the periodicity in the 


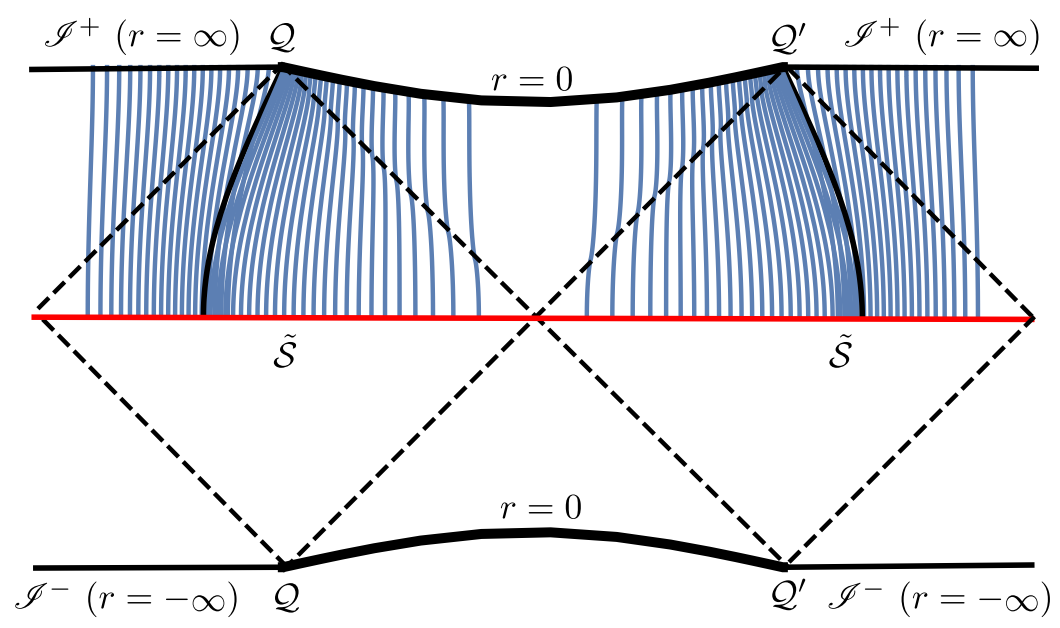

Figure 5: Numerical computation of the congruence of conformal geodesics given by Theorem 1 in the Penrose diagram of the Schwarzschild-de Sitter spacetime for $M=1 / 3$. The curves have been computed numerically using Mathematica. The critical curves described by the condition $r=r_{\circledast}$ are depicted as solid black curves reaching the asymptotic points $\mathcal{Q}$ and $\mathcal{Q}^{\prime}$. Notice that the congruence is initially orthogonal to the initial hypersurface $\tilde{\mathcal{S}}$.

spatial direction of the maximal extension of the spacetime, it is only necessary to restrict the discussion to the range $r_{b} \leq r_{\star} \leq r_{c}$.

In what follows let denote by $\operatorname{SdS}_{I}$ the region in the conformal representation of the subextremal Schwarzschild-de Sitter spacetime defined by the conformal factor $\Theta$ associated to the congruence of conformal geodesics which is bounded by the critical conformal geodesic and the conformal geodesic passing by the bifurcation sphere of the Cosmological horizon - boundaries included. Similarly, we define $\operatorname{SdS}_{I I}$ as the region bounded by the critical geodesic and the geodesic passing through the bifurcation sphere of the black hole horizon - we exclude the former part of the boundary and include the latter.

The region $\mathbf{S d S}_{I}$. For $r \in(0, \infty)$ and $u \in(-\infty, \infty)$, let $z \equiv 1 / r$ and $w \equiv \tanh u$. In terms of these coordinates one has that

$$
\operatorname{SdS}_{I}=\left\{z \in\left[0, z_{\circledast}\right], w \in[-1,1]\right\} .
$$

The analysis of the previous Sections shows that the map

$$
(w, z):[0,2] \times\left[r_{\circledast}, r_{c}\right] \longrightarrow[-1,1] \times\left[0, z_{\circledast}\right]
$$

with

$$
w=\tanh u\left(\tau, r_{\star}\right), \quad z=1 / r\left(\tau, r_{\star}\right)
$$

as defined by the solutions to the conformal geodesics depends analytically on its parameters. The analysis of the conformal geodesic deviation equation implies that the Jacobian of the transformation is non-zero for the given value of the parameters - it can be readily verified that the function $\Theta \tilde{\omega}$ coincides with the Jacobian. Thus, it follows that the inverse map

$$
\left(\tau, r_{\star}\right):[-1,1] \times\left[0, z_{\circledast}\right] \longrightarrow[0,2] \times\left[r_{\circledast}, r_{c}\right]
$$

with

$$
\tau=\tau(\operatorname{arctanh} w, 1 / z), \quad r_{\star}=r_{\star}(\operatorname{arctanh} w, 1 / z),
$$

and $u \in \mathbb{R}$ is well defined and also an analytic function of its parameters. Thus, ignoring angular coordinates, this inverse map defines a conformal Gaussian system of coordinates in $\operatorname{SdS}_{I}$. In particular, given any point in $\mathrm{SdS}_{I}$, there is a unique conformal geodesic passing through it. Thus, the congruence of conformal geodesics covers the whole of $S d S_{I}$. 


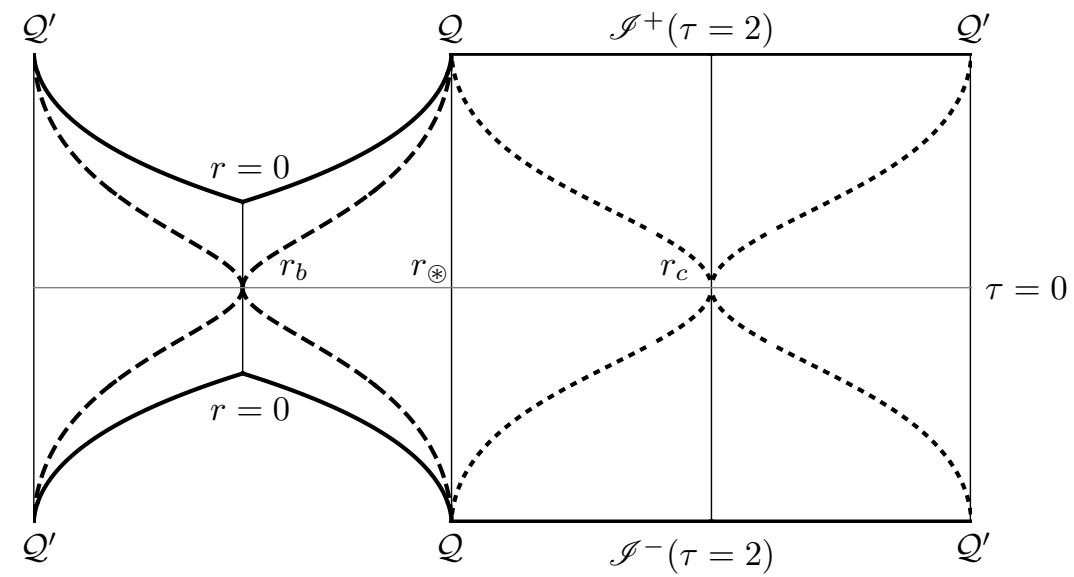

Figure 6: The Schwarzschild-de Sitter spacetime in conformal Gaussian coordinates $\left(r_{\star}, \tau\right)$ for $M=1 / 3$. The horizontal line represents the initial hypersurface $\tilde{\mathcal{S}}$ which is parametrised by $r_{\star}$. The vertical axis corresponds to the unphysical proper time $\tau$. In particular, the location of the black hole horizon in conformal Gaussian coordinates, $\left(r_{b}, \tau\left(r_{b}, r_{\star}\right)\right)$ with $r_{b} \leq r_{\star} \leq r_{\circledast}$, corresponds to the dashed curve. The thick continuous line denotes the location of the singularity $\left(0, \tau\left(0, r_{\star}\right)\right)$ with $0 \leq r_{\star} \leq r_{\circledast}$. Similarly, the location of the cosmological horizon in conformal Gaussian coordinates, $\left(r_{c}, \tau\left(r_{c}, r_{\star}\right)\right)$ with $r_{\circledast} \leq r_{\star} \leq r_{c}$, is depicted in the dotted curve. The vertical lines correspond to the conformal geodesics starting at the bifurcation spheres, $r_{\star}=r_{b}$ and $r_{\star}=r_{c}$ respectively, and the critical conformal geodesic $r_{\star}=r_{\circledast}$. The future and past conformal boundary $\mathscr{I}^{ \pm}$are located at $\tau= \pm 2$ which are reached by those conformal geodesics with initial datum $r_{\circledast}<r_{\star}<r_{c}$.

The region $\operatorname{SdS}_{I I}$. Let $y=\tanh v$. In terms of the coordinates $(y, r)$ the region $\operatorname{SdS}_{I I}$ is described as

$$
\mathrm{SdS}_{I I}=\left\{r \in\left(0, r_{\circledast}\right), y \in[-1,1]\right\} .
$$

As with the region $\mathrm{SdS}_{I}$, the analysis from the previous Sections shows that the map

$$
(y, r):\left[0, \tau_{\text {夕 }}\right) \times\left[r_{b}, r_{\circledast}\right] \longrightarrow[-1,1] \times\left(0, r_{\circledast}\right]
$$

is well defined and an analytic function of its parameters. The analysis of the conformal geodesic deviation equation implies that the Jacobian of the transformation, given by the function $\tilde{\omega}$, is non-zero for the given value of the parameters. Thus, it follows that the inverse map

$$
\left(\tau, r_{\star}\right):[-1,1] \times\left(0, r_{\circledast}\right] \longrightarrow\left[0, \tau_{\text {夕夕 }}\right) \times\left[r_{b}, r_{\circledast}\right]
$$

is well defined and also an analytic function of its parameters. Thus, ignoring angular coordinates, this inverse map defines a conformal Gaussian system of coordinates in $\mathrm{SdS}_{I I}$. In particular, the congruence of conformal geodesics covers the whole of $S d S_{I I}$.

\subsection{Summary of the analysis}

The analysis of Section 4 can be summarised in the following proposition.

Theorem 1 (Conformal geodesics in the subextremal Schwarzschild-de Sitter spacetime). The maximal extension of the subextremal Schwarzschild-de Sitter spacetime can be covered by a non-singular congruence of conformal geodesics. The existence of this congruence implies the existence of a global system of conformal Gaussian coordinates in the spacetime.

A qualitatively accurate depiction of the congruence of conformal geodesics in the Penrose diagram of the Schwarzschild-de Sitter spacetime can be seen in Figure 5 . 


\section{Analysis of the conformal geodesics in the extremal Schwarzschild-de Sitter case}

In this Section, for concreteness we restrict our discussion to the representation of the extremal Schwarzschild-de Sitter spacetime as a whitehole so that there the singularity lies in the past and the conformal boundary in the future - see Figure 2 (a).

Throughout this Section it is assumed that $M=2 /(3 \sqrt{3})$. In this case, $D(r)$ vanishes at $r_{\mathcal{H}} \equiv 1 / \sqrt{3}$ while $D(r)<0$ for $r_{\mathcal{H}} \in \mathbb{R}^{+} /\{1 / \sqrt{3}\}$. Hence for $r \in \mathbb{R}^{+} /\{1 / \sqrt{3}\}$, the coordinate $r$ is a time coordinate while $t$ is a spatial one. This means that the hypersurfaces $r=r_{\star}$ with $r_{\star} \neq r_{\mathcal{H}}$ are spacelike while those of constant $t$ are timelike - see Figure 4. Consequently, one purports to construct a congruence of conformal geodesics by prescribing initial data on hypersurfaces of constant $r=r_{\star}$ with $r_{\star} \neq 0$ and $r_{\star} \neq r_{\mathcal{H}}$. In addition, observe that the critical curve located at $r_{\circledast}$ as defined in Section 4.2.1 coincides in this case with the location of the Killing horizon $r_{\mathcal{H}}$.

\subsection{Basic setup}

Similar to the subextremal case we set

$$
\Theta_{\star}=1 .
$$

Hence $\tilde{\boldsymbol{\beta}}_{*}=\Theta_{\star}^{-1} \mathbf{d} \Theta_{\star}=0$ and we get $\beta_{\star}=0$. This means that $\beta=0$ since $\beta$ is a constant along a given conformal geodesic. As in the case of Section 4, this choice simplifies the equations for the curve as these reduce to the equations of metric geodesics. In the remainder of this Section let

$$
\tilde{\mathcal{S}} \equiv\left\{r=r_{\star}\right\}
$$

with $r_{\star}$ a constant $r_{\star} \neq 0$ and $r_{\star} \neq 1 / \sqrt{3}$ —see Figure 7 As already pointed out, the above hypersurface of the extremal Schwarzschild-de Sitter spacetime is spacelike. Particularising equations (41) and 38 for the case $\beta=0$ we get

$$
r_{\star}^{\prime}=\sqrt{\gamma^{2}-D_{\star}}, \quad t_{\star}^{\prime}=\frac{|\gamma|}{\left|D_{\star}\right|} .
$$

Remark 13. Notice that in the extremal case $D_{\star}<0$ and in contrast with the initial data chosen for the subextremal case the congruence is not necessarily orthogonal to the initial hypersurface $\tilde{\mathcal{S}}$. To see this more clearly, observe that the unit normal to $\tilde{\mathcal{S}}$ is given by $\boldsymbol{n}=\left(1 / \sqrt{\left|D_{\star}\right|}\right) \mathbf{d} r$ while $\tilde{\boldsymbol{x}}_{\star}^{\prime}=r_{\star}^{\prime} \boldsymbol{\partial}_{r}+t_{\star}^{\prime} \boldsymbol{\partial}_{t}$. In particular, notice that $\boldsymbol{n}^{\sharp}$ and $\tilde{\boldsymbol{x}}_{\star}^{\prime}$ are not parallel and that $\left\langle\boldsymbol{n}, \tilde{\boldsymbol{x}}_{\star}^{\prime}\right\rangle=$ $\sqrt{\gamma^{2} /\left|D_{\star}\right|+1}$. Compare this with the discussion for the subextremal Schwarzschild-de Sitter spacetime given in Section 4 where $D_{\star}>0$ and the symmetry of the spacetime suggested to consider the initial hypersurface determined by the condition $t=0$ which has unit normal $\boldsymbol{n}=$ $\sqrt{D_{\star}} \mathbf{d} t$. In this case, setting $\gamma=\sqrt{D_{\star}}$ leads to the initial data $\sqrt[51]{5}$ for which $\tilde{\boldsymbol{x}}_{\star}^{\prime}$ and $\boldsymbol{n}^{\sharp}$ coincide - see Figure 4. In the extremal case however there is not a apriori preferred choice for $r_{\star} \neq r_{\mathcal{H}}$ determining the initial hypersurface — see Figure 7. Consequently, instead of prescribing data corresponding to a congruence starting orthogonal to $\tilde{\mathcal{S}}$ we will consider $\gamma$ as a free parameter so that, in general, the congruence can start oblique to the initial hypersurface.

Remark 14. Observe that for $r_{\star}<r_{\mathcal{H}}$ the hypersurface $\tilde{\mathcal{S}}$ is below the horizon while if $r_{\star}>r_{\mathcal{H}}$ then it lies above it. Our analysis will consider both situations simultaneously.

\subsubsection{Conformal factor}

Since $\beta=0$ one has that the 1 -form $\tilde{\boldsymbol{\beta}}$ vanishes. Using equation 22 and $(24)$ one observes that $\boldsymbol{\beta}=\dot{\Theta} \dot{\boldsymbol{x}}^{b}$. Taking into account equation $(96)$ along with the constraints 20 one gets

$$
\ddot{\Theta}_{\star}=\frac{1}{2} \dot{\Theta}_{\star}^{2}-\frac{1}{6} \lambda
$$

For conciseness and consistency with the notation of Section 2 one sets $\lambda=-3 \epsilon$. For the case of a de-Sitter like cosmological constant one sets $\epsilon=-1$. Moreover, without loss of generality let 


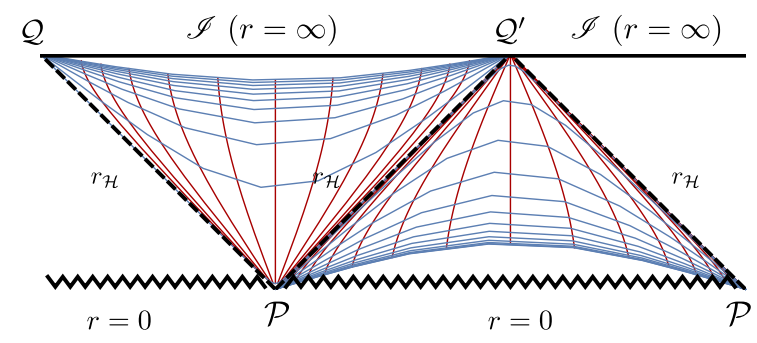

Figure 7: Curves with constant $t$ and $r$ (red and blue respectively) are plotted on the Penrose diagram of the extremal Schwarzschild-de Sitter spacetime. In contrast with the subextremal case curves with constant $t$ in starting from some $r_{\star}<r_{\mathcal{H}}$ accumulate at the asymptotic points $\mathcal{Q}$ and $\mathcal{Q}^{\prime}$ while those starting from $r_{\star}>r_{\mathcal{H}}$ accumulate at $\mathcal{P}$.

us set $\tau_{\star}=0$, then the conformal factor associated to the congruence defined by the initial data discussed in Section 5.1 is found by using the general expression 19

$$
\Theta(\tau)=1+\dot{\Theta}_{\star} \tau+\frac{1}{4}\left(\dot{\Theta}_{\star}^{2}-1\right) \tau^{2},
$$

where $\dot{\Theta}_{\star}=\left\langle\boldsymbol{\beta}_{\star}, \dot{\boldsymbol{x}}_{\star}\right\rangle$. The roots of $\Theta(\tau)$ are given by

$$
\tau_{+}=\frac{2}{1-\dot{\Theta}_{\star}} \quad \tau_{-}=-\frac{2}{1+\dot{\Theta}_{\star}} .
$$

Although one has formally two roots of the conformal factor $\Theta$, an inspection of the conformal diagram of Figure 7 reveals that only one component of the conformal boundary is actually realised. Moreover, in order to have $\tau_{ \pm}$finite, we restrict the possible values of $\dot{\Theta}_{\star}$ to those that satisfy $\left|\dot{\Theta}_{\star}\right| \neq 1$. This corresponds to restrict our analysis to compact conformal representations in which $\mathscr{I}$ is located at a finite value of the unphysical proper time $\tau$.

The relation between the unphysical proper time $\tau$ and the physical proper time $\tilde{\tau}$ is readily obtained form equation (21):

$$
\tilde{\tau}=2 \operatorname{arctanh}\left(\frac{1}{2}\left(1-\dot{\Theta}_{\star}^{2}\right) \tau-\dot{\Theta}_{\star}\right)+2 \operatorname{arctanh} \dot{\Theta}_{\star} .
$$

Therefore,

$$
\tau=\frac{2}{1-\dot{\Theta}_{\star}^{2}} \tanh (\tilde{\tau} / 2)+\frac{2 \dot{\Theta}_{\star}}{1-\dot{\Theta}_{\star}^{2}} .
$$

From these expressions we deduce that $\lim _{\tilde{\tau} \rightarrow \pm \infty} \tau=\tau_{ \pm}$. For conciseness let us take $0<\dot{\Theta}_{\star}<1$ so that $\tau_{+}>0$ and $\tau_{-}<\tau_{+}$. Using equation $(99)$ we see that the conformal factor can be rewritten in terms of the $\tilde{\boldsymbol{g}}$-proper time $\tilde{\tau}$ as

$$
\Theta(\tilde{\tau})=\frac{1}{1-\dot{\Theta}_{\star}^{2}} \operatorname{sech}^{2}(\tilde{\tau} / 2) .
$$

\subsubsection{Initial conditions for the congruence}

Unlike in the other cases studied in this article, we do not require the congruence to be everywhere orthogonal to the initial hypersurface $\tilde{\mathcal{S}}$. However, we are interested in showing that the curves arrive orthogonally to the conformal boundary. To do so we first compute

$$
\mathbf{d} \Theta=\left(\dot{\Theta}_{\star}+\frac{1}{2}\left(\dot{\Theta}_{\star}^{2}-1\right) \tau\right) \mathbf{d} \tau .
$$

Observe that $\mathbf{d} \Theta=\mathbf{0}$ at $\tau=2 \dot{\Theta}_{\star} /\left(1-\dot{\Theta}_{\star}^{2}\right)$. Hence, $\mathbf{d} \Theta \neq \mathbf{0}$ at $\mathscr{I}$. Now from 21) on has that

$$
\mathbf{d} \tau=\Theta \mathbf{d} \tilde{\tau} .
$$


Using equation 41 one can consider $r=r(\tilde{\tau})$, equivalently $\tilde{\tau}=\tilde{\tau}(r)$, therefore $\mathbf{d} \tilde{\tau}=\left(1 / r^{\prime}\right) \mathbf{d} r$. Using the last observation and equations (101) and 102 and one gets

$$
\mathbf{d} \Theta=\Theta\left(\dot{\Theta}_{\star}+\frac{1}{2}\left(\dot{\Theta}_{\star}^{2}-1\right) \tau\right) \frac{1}{r^{\prime}} \mathbf{d} r .
$$

Recall now that, as described in Section 3.5. we are considering geodesic equations with no evolution in the angular coordinates, thus

$$
\tilde{\boldsymbol{x}}^{\prime}=t^{\prime} \partial_{t}+r^{\prime} \boldsymbol{\partial}_{r}
$$

since one is effectively analysing the curves on a 2-dimensional manifold $\tilde{\mathcal{M}} / S O(3)$ with metric $\tilde{\boldsymbol{l}}$ as given in equation (36). Therefore, one can define a unique orthogonal vector to $\tilde{\boldsymbol{x}}^{\prime}$ by $\tilde{\boldsymbol{x}}^{\prime \perp b} \equiv \tilde{\boldsymbol{\epsilon}}_{\tilde{l}}\left(\tilde{\boldsymbol{x}}^{\prime}, \cdot\right)$. A direct computation shows that

$$
\tilde{\boldsymbol{x}}^{\prime \perp b}=-r^{\prime} \mathbf{d} t+t^{\prime} \mathbf{d} r,
$$

so that,

$$
\tilde{\boldsymbol{x}}^{\perp}=-\frac{r^{\prime}}{D(r)} \boldsymbol{\partial}_{t}-t^{\prime} D(r) \boldsymbol{\partial}_{r} .
$$

Hence, using (22) one has that

$$
\dot{\boldsymbol{x}}^{\perp}=-\frac{1}{\Theta}\left(\frac{r^{\prime}}{D(r)} \boldsymbol{\partial}_{t}+t^{\prime} D(r) \boldsymbol{\partial}_{r}\right) .
$$

Therefore using equations 103, 104, 411 and 42 we get

$$
\left\langle\mathbf{d} \Theta, \dot{\boldsymbol{x}}^{\perp}\right\rangle=\frac{|\gamma|}{\sqrt{\gamma^{2}-D(r)}}\left(\dot{\Theta}_{\star}+\frac{1}{2}\left(\dot{\Theta}_{\star}^{2}-1\right) \tau\right) .
$$

Taking into account that $\lim _{\tilde{r} \rightarrow \infty} D(r)=-\infty$ and that

$$
\dot{\Theta}_{\star}+\frac{1}{2}\left(\dot{\Theta}_{\star}^{2}-1\right) \tau_{ \pm}=\mp 1,
$$

and then assuming $\gamma \neq 0$ one concludes

$$
\left\langle\mathbf{d} \Theta, \dot{\boldsymbol{x}}^{\perp}\right\rangle=0 \text { at } \mathscr{I} .
$$

This means that the curves arrive orthogonally to the conformal boundary.

Remark 15. A quick inspection of the last argument shows that one can perform a similar calculation for the congruence of conformal geodesics in the subextremal Schwarzschild-de Sitter spacetime of Section 4 and obtain the same conclusion.

Remark 16. This is in agreement with Proposition 3.1 given in [7] where it is shown that in an Einstein spacetime, any conformal geodesic leaving $\mathscr{I}$ orthogonally is up to reparametrisation a geodesic of $\tilde{\boldsymbol{g}}$.

\subsection{Qualitative analysis of the behaviour of the curves}

In this Section we carry out a qualitative study of the family of curves in the extremal Schwarzschildde Sitter analogous to that of Section 4.2 for the subextremal case. We distinguish three basic types of curves: those escaping to the asymptotic points and those emanating from the singularity in the past and escaping to the conformal boundary in the future. 


\subsubsection{Conformal geodesics with constant $t$}

The condition determining the location of the curves with constant $r$ is given by

$$
\gamma^{2}-D(r)=0
$$

Since $D(r) \leq 0$ and given that $D(r)$ only vanishes for positive $r$ at $r=r_{\mathcal{H}}$ then, the critical curve is characterised by the conditions $\gamma=0$ and $r=r_{\mathcal{H}}$. This is consistent with the analysis of Section 4.2.1 since $r_{\circledast}$ as given in equation $\left(59\right.$ reduces to $r_{\mathcal{H}}=1 / \sqrt{3}$ for $M=2 / 3 \sqrt{3}$. Moreover, notice that by virtue of equation 42 and the initial data given in Section 5.1 conformal geodesics with $\gamma=0$ and $r \neq r_{\mathcal{H}}$ coincide with curves of constant $t$. These curves accumulate at the asymptotic points $\mathcal{Q}$ and $\mathcal{Q}^{\prime}$ - see Figure 7. Observe that for $\gamma=0$ and $\beta=0$ the expression 43a can be explicitly integrated to yield

$$
\tilde{\tau}=r_{\mathcal{H}} \ln \left(H(r) / H\left(r_{\star}\right)\right)
$$

where

$$
H(r) \equiv\left(\frac{\sqrt{3 r}+\sqrt{r+2 r_{\mathcal{H}}}}{\left(\sqrt{3 r}-\sqrt{r+2 r_{\mathcal{H}}}\right)\left(\sqrt{r}+\sqrt{r+2 r_{\mathcal{H}}}\right)^{2 \sqrt{3}}}\right) .
$$

Observe that equation (105), as pointed out in [15], implies that the geodesics with $\gamma=0$ never cross the horizon since $\tilde{\tau} \rightarrow \infty$ as $r \rightarrow r_{\mathcal{H}}$. Using equation $(99)$ and setting $\dot{\Theta}_{\star}=0$ for simplicity, we obtain

$$
\tau(r)=2 \frac{W(r)-W_{\star}}{W(r)+W_{\star}}
$$

where $W(r)=H(r)^{r_{\mathcal{H}}}$.

Asymptotic behaviour of the curve. To analyse the behaviour of these curves as they approach the asymptotic points $\mathcal{Q}$ and $\mathcal{Q}^{\prime}$ let us consider $r=r_{\mathcal{H}}+\epsilon$. Then one obtains that for small $\epsilon>0$ that

$$
W(r)=\left(\frac{C_{1}}{\epsilon}\right)^{r \mathcal{H}}\left(C_{2}+C_{3} \epsilon+\mathcal{O}\left(\epsilon^{2}\right)\right)
$$

where $C_{1}, C_{2}, C_{3}$ are numerical factor not relevant for the subsequent discussion. To leading order $W(r)=C / \epsilon^{p}$ where we have used the value of $r_{\mathcal{H}}$ and introduced $p=1 / \sqrt{3}$ and $C=C_{1}^{r \mathcal{H}} C_{2}$ to simplify the notation. Consequently, to leading order we have

$$
\frac{\mathrm{d} \tau}{\mathrm{d} \epsilon}=\frac{-4 W_{\star} C p \epsilon^{p-1}}{\left(C+W_{\star} \epsilon^{p}\right)^{2}} .
$$

Since $p<1$ then one concludes that $\mathrm{d} \tau / \mathrm{d} \epsilon$ diverges as $\epsilon \rightarrow 0$. Therefore the curves with $\gamma=0$ become tangent to the Killing horizon as the approach the asymptotic points $\mathcal{Q}$ and $\mathcal{Q}^{\prime}$. In other words, as in the subextremal case, these curves become asymptotically null.

\subsubsection{Conformal geodesics with non-constant $r$}

Recalling that

$$
r^{\prime}=\sqrt{\gamma^{2}-D(r)}
$$

and observing that $D(r) \leq 0$, it follows that if $r_{\star}^{\prime} \neq 0$ then, in fact, $r^{\prime}>0$. Moreover, one can show that $r_{\star}^{\prime \prime}>0$ and that $r^{\prime \prime} \neq 0$ for $r \in\left[r_{\star}, \infty\right)$. Thus, the curves escape to the conformal boundary.

Behaviour towards the conformal boundary. We now show that the congruence of conformal geodesics reaches the conformal boundary $\mathscr{I}$ in an infinite amount of physical proper time. In order to see this, first observe from equation (12) that $D(r) \leq 0$, consequently from equation (41) it follows that $r(\tilde{\tau})$ is a monotonic function. Moreover, using equations $(12)$ and $(42)$ we find that

$$
\tilde{\tau}=\int_{r_{\star}}^{r} \frac{\mathrm{d} \bar{r}}{\sqrt{\gamma^{2}+\frac{1}{\bar{r}}\left(\frac{2}{\sqrt{3}}+\bar{r}\right)\left(\bar{r}-\frac{1}{\sqrt{3}}\right)^{2}}} .
$$


We are interested in analysing the convergence of

$$
\tilde{\tau}_{\infty} \equiv \int_{r_{\star}}^{\infty} \frac{1}{\sqrt{\gamma^{2}+\frac{1}{\bar{r}}\left(\frac{2}{\sqrt{3}}+\bar{r}\right)\left(\bar{r}-\frac{1}{\sqrt{3}}\right)^{2}}} \mathrm{~d} \bar{r} .
$$

Introducing a new variable $\xi \equiv r-1 / \sqrt{3}$ then we can rewrite the integral as

$$
\tilde{\tau}_{\infty} \equiv \int_{\xi_{\star}}^{\infty} \frac{1}{\sqrt{\gamma^{2}+\frac{\bar{\xi}^{2}(\bar{\xi}+\sqrt{3})}{\bar{\xi}+\frac{1}{\sqrt{3}}}}} \mathrm{~d} \bar{\xi} .
$$

Since $r \geq r_{\star}>0$ one has $r \geq \delta_{\star}$ for some $\delta_{\star}>0$ small. Thus $\xi \geq-1 / \sqrt{3}+\delta_{\star}$. Therefore we have that

$$
\frac{\xi+\sqrt{3}}{\xi+\frac{1}{\sqrt{3}}} \leq \kappa^{2} \quad \text { with } \quad \kappa^{2} \equiv 1+\frac{2}{\sqrt{3} \delta_{\star}} .
$$

Using the latter, we have that

$$
\sqrt{\gamma^{2}+\frac{\xi^{2}(\xi+\sqrt{3})}{\xi+\frac{1}{\sqrt{3}}}} \leq \sqrt{\gamma^{2}+\kappa^{2} \xi}
$$

so that

$$
\tilde{\tau}_{\infty} \geq \int_{\xi_{\star}}^{\infty} \frac{1}{\sqrt{\gamma^{2}+\kappa^{2} \bar{\xi}^{2}}} \mathrm{~d} \bar{\xi}
$$

This last integral diverges and one concludes that $\tilde{\tau}_{\infty}$ diverges as well. Accordingly, the conformal boundary is reached in an infinite amount of physical proper time.

Remark 17. For initial hypersurfaces with $r_{\star}<r_{\mathcal{H}}$ (i.e. lying below the horizon), a direct inspection of the integral in

$$
\tilde{\tau}_{\mathcal{H}}=\int_{r_{\star}}^{r_{\mathcal{H}}} \frac{\mathrm{d} s}{\sqrt{\gamma^{2}+\frac{1}{s}\left(\frac{2}{\sqrt{3}}+s\right)\left(s-\frac{1}{\sqrt{3}}\right)^{2}}},
$$

shows that it is finite - there are no zeros in the denominator for $s \in\left[r_{\star}, r_{\mathcal{H}}\right]$. Thus the horizon is reached in a finite amount of proper time.

Behaviour towards the singularity. We now shown that both the horizon and the singularity are reached by the curves of the congruence in a finite amount of physical proper time $\tilde{\tau}$. This behaviour is a consequence of the fact that the integral in equation 107) is finite for any $r$ with $0 \leq r \leq r_{\star}$. To prove this we start from the inequality

$$
\sqrt{\gamma^{2}+\frac{1}{r}\left(\frac{2}{\sqrt{3}}+r\right)\left(r-\frac{1}{\sqrt{3}}\right)^{2}} \geq|\gamma|
$$

Therefore, for $\gamma \neq 0$ this entails

$$
\tilde{\tau}=\int_{r_{\star}}^{r} \frac{\mathrm{d} \bar{r}}{\sqrt{\gamma^{2}+\frac{1}{\bar{r}}\left(\frac{2}{\sqrt{3}}+\bar{r}\right)\left(\bar{r}-\frac{1}{\sqrt{3}}\right)^{2}}} \leq \int_{r_{\star}}^{r} \frac{\mathrm{d} \bar{r}}{|\gamma|}=\frac{r-r_{\star}}{|\gamma|} .
$$

This last inequality shows the assertion. In what follows we denote by $\tilde{\tau}_{\xi}$ (respectively $\tau_{\not}$ ) the value of the proper time at which the singularity is reached. 


\subsubsection{Behaviour of the congruence using null coordinates}

Most of the analysis performed for the asymptotic region $r>r_{c}$ in the subextremal case in Section 4.2 .2 can also be applied for the extremal case for any $r>r_{\star}>0$ since in this case one has $D(r) \leq 0$ then the expression

$$
\sqrt{\gamma^{2}-D(r)} \geq|\gamma|
$$

is valid for any $r$. This leads to formally the same estimates as in Section 4.2.2. Notice however that the congruence does not start orthogonally to the initial hypersurface $\mathcal{S}$. Assuming $\gamma \neq 0$ and performing formally the same procedure leading to equation $(76)$ we get

$$
\left|u(r)-u_{\star}\right| \leq \frac{r-r_{\star}}{\gamma^{2}} .
$$

This estimate is valid for any $r \geq r_{\star}>0$. In particular observe that $\left|u\left(r_{\mathcal{H}}\right)-u_{\star}\right|$ is finite. Now, let us denote as before $u_{\infty} \equiv \lim _{r \rightarrow \infty} u$ and take $r_{\mathcal{H}}<r_{\bullet}<\infty$, then the same procedure leading to 77 renders

$$
\left|u_{\infty}-u\left(r_{\bullet}\right)\right| \leq \int_{r_{\bullet}}^{\infty} \frac{2}{|D(\bar{r})|} \mathrm{d} \bar{r} .
$$

In contrast to Section 4.2.2, at this point one can compute the last integral using the explicit functional form for $D(r)$ in the extremal case:

$$
\left|u_{\infty}-u\left(r_{\bullet}\right)\right| \leq \frac{6}{r_{\bullet}-r_{\mathcal{H}}}-\frac{4}{\sqrt{3}} \ln \left(\frac{r_{\bullet}-r_{\mathcal{H}}}{r_{\bullet}+r_{\mathcal{H}}}\right) \equiv u_{\circ} .
$$

Observe that since $r_{\mathcal{H}}<r_{\bullet}<\infty$ one has that $0<u_{\circ}<\infty$. Finally, using expressions 108, (109) and the triangle inequality render

$$
\left|u_{\infty}-u_{\star}\right| \leq\left|u_{\infty}-u\left(r_{\bullet}\right)\right|+\left|u\left(r_{\bullet}\right)-u_{\star}\right|<u_{\circ}+\frac{r_{\bullet}-r_{\star}}{\gamma^{2}} .
$$

Remark 18. It follows then that the conformal geodesics cross the horizon and escape the conformal boundary with a finite value of the retarded null time $u$. Thus, they remain away from the asymptotic points $\mathcal{Q}$ and $\mathcal{Q}^{\prime}$.

Remark 19. An analogous analysis can be carried out with the advanced null coordinate $v$.

\subsection{Explicit expressions in terms of elliptic functions}

As in the subextremal case the solutions to the conformal geodesic equations can be written in terms of elliptic functions. We begin by observing that, using the functional form of $D(r)$ as given in equation 12 , one can rewrite

$$
\gamma^{2}-D(r)=\frac{1}{r}\left(r^{3}+\left(\gamma^{2}-1\right) r+\frac{2}{3 \sqrt{3}}\right) .
$$

One can verify that the discriminant of the cubic $r^{3}+\left(\gamma^{2}-1\right) r+2 / 3 \sqrt{3}$ is always negative provided that $\gamma \neq 0$. Therefore one can factorise the above expression as

$$
\gamma^{2}-D(r)=\frac{1}{r}\left(r-\delta_{+}\right)(r-\delta)(r-\bar{\delta}),
$$

where $\delta_{+}>0$ while $\delta$ and $\bar{\delta}$ are complex conjugate. Consequently, setting $\beta=0$ the integral 43a) can be written as

$$
\tilde{\tau}=\int_{r_{\star}}^{r} \sqrt{\frac{s}{\left(s-\delta_{+}\right)(s-\delta)(r-\bar{\delta})}} \mathrm{d} \bar{s} .
$$

Curves with $\gamma=1$. Rather than considering the previous expression for an arbitrary nonvanishing value of the constant of integration $\gamma$, we now particularise to the case $\gamma=1$. This 


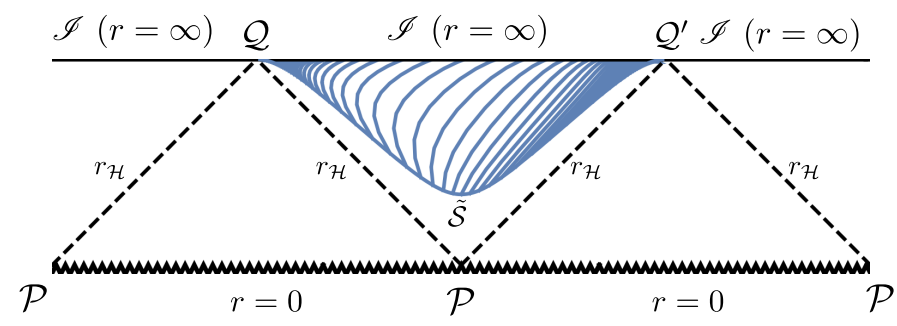

Figure 8: Plot of a numerical simulation of a congruence of conformal geodesics on the Penrose diagram of the extremal Schwarzschild-de Sitter maximal extension. For this simulation $\gamma=1 / 55$ and the initial hypersurface $\tilde{\mathcal{S}}$ is given by $r=0.7>1 / \sqrt{3}$ so it is outside the black hole region and hence the curves go to null infinity.

choice leads to simpler explicit expressions and can be done irrespectively of the value of $r_{\star}$. For $\gamma=1$ a direct computation yields

$$
\tilde{\tau}=\frac{1}{6 \sqrt[3]{2} r_{\mathcal{H}}}\left(2 \sqrt{3} \arctan \left(\frac{\sqrt[3]{4} r-r_{\mathcal{H}}}{\sqrt{3} r_{\mathcal{H}}}\right)-2 \ln \left|\sqrt[3]{4} r+2 r_{\mathcal{H}}\right|+\ln \left|\sqrt[3]{2} r^{2}-\sqrt[3]{4} r_{\mathcal{H}} r+2 r_{\mathcal{H}}^{2}\right|\right)+c_{\star}
$$

where $c_{\star}$ is an integration constant and one can verify that $\sqrt[3]{2} r^{2}-\sqrt[3]{4} r r_{\mathcal{H}}+2 r_{\mathcal{H}}^{2}$ never vanishes. Thus, $\tilde{\tau}$ as given by the above expression is an analytic function of its arguments.

For the extremal Schwarzschild-de Sitter spacetime it is not possible to construct Kruskal-like coordinates. However, one can still construct null coordinates $u$ and $v$ using the tortoise radial coordinate $r$. A straightforward computation shows that the tortoise coordinate in this case is given by

$$
r(r)=\frac{r_{\mathcal{H}}}{\left(r-r_{\mathcal{H}}\right)\left(r+\left|r_{-}\right|\right)}-\frac{\left|r_{-}\right|}{\left(r_{\mathcal{H}}+\left|r_{-}\right|\right)^{2}} \ln \left|\frac{r-r_{\mathcal{H}}}{r+\left|r_{-}\right|}\right| .
$$

Observe that

$$
\lim _{r \rightarrow \infty} f(r)=0
$$

Similarly, one can show that, taking the limit as $r$ approaches $r_{\mathcal{H}}$ from the left $\left(r<r_{\mathcal{H}}\right)$ one has

$$
\lim _{r \rightarrow r_{\mathcal{H}}^{-}} f(r)=-\infty
$$

while taking the limit from the right $\left(r>r_{\mathcal{H}}\right)$ one obtains

$$
\lim _{r \rightarrow r_{\mathcal{H}}^{+}} f(r)=\infty
$$

Taking into account expressions (111) and (112) and proceeding as in Section 4.2.4 one concludes that $u(\tau)$ and $v(\tau)$ are also analytic functions or their parameters. As previously discussed, in the extremal case the critical curve is characterised by the conditions $t=t_{\star}$ and $r=r_{\mathcal{H}}$. Using equation (6) and the limits (113)-(114) one concludes that at the asymptotic points $\mathcal{Q}$ and $\mathcal{Q}^{\prime}$ one has respectively $u=\infty$ and $u=-\infty$.

\subsection{Analysis of the conformal geodesic deviation equation}

Given that for the initial data for the congruence we have set $\beta=0$, it follows that the scalar $\tilde{\omega}$ describing the deviation of the congruence satisfies equation (94).

\subsubsection{Initial data for the deviation equation}

As previously discussed, one important difference between the extremal and the subextremal case is that in the former case $r$ is a time coordinate while $t$ is a spatial coordinate everywhere - thus, $\partial_{t}$ is a spatial vector. If $r_{\star}^{\prime}>0$ the conformal geodesics end at the conformal boundary, while if 
$r_{\star}^{\prime}<0$ the conformal geodesics end at the singularity. In the former case a suitable choice for the initial value of the deviation vector is $\tilde{\boldsymbol{z}}_{\star}=-\boldsymbol{\partial}_{t}$. Proceeding in similar way as in Section 3.7 one gets $\tilde{\omega}_{\star}=r_{\star}^{\prime}>0$ and $\tilde{\omega}_{\star}^{\prime}=0$ if one wants to analyse the behaviour of the congruence towards the conformal boundary. To analyse the behaviour towards the singularity it is convenient to set $\boldsymbol{z}_{\star}=\boldsymbol{\partial}_{t}$ so that $\tilde{\omega}_{\star}=-r_{\star}^{\prime}>0$. In any case we can write $\tilde{\omega}_{\star}=\left|r_{\star}^{\prime}\right|$ which allow us to discuss both cases simultaneously.

\subsubsection{Estimating the solution to the deviation equation}

From equation (94), one readily obtains the estimate

$$
\frac{\mathrm{d}^{2} \tilde{\omega}}{\mathrm{d} \tilde{\tau}^{2}}>\tilde{\omega}
$$

Proceeding in an analogous way as in Section 4.4 .2 we get

$$
\tilde{\omega}>\left|r^{\prime}\right|_{\star} \cosh (\tilde{\tau})>\left|r_{\star}^{\prime}\right| .
$$

From the last expression it follows that for the case $r_{\star}^{\prime}<0$ in which the conformal geodesics end at the singularity that $\tilde{\omega} \neq 0$ so that there are no conjugate points in the congruence. In the case $r_{\star}^{\prime}>0$ where the curves escape to the conformal boundary, one can proceed as follows: Using equation 100 one obtains

$$
\omega(\tilde{\tau})=\Theta(\tilde{\tau}) \tilde{\omega}(\tilde{\tau})>\frac{r_{\star}^{\prime}}{1-3 \dot{\Theta}_{\star}} \operatorname{sech}^{2}(\tilde{\tau} / 2) \cosh (\tilde{\tau}) .
$$

Notice that the expression on the right hand side of equation 115 is always finite, moreover

$$
\omega(\tilde{\tau})>\left(\frac{\left|r_{\star}^{\prime}\right|}{1-3 \dot{\Theta}_{\star}}\right) \frac{2 \cosh (\tilde{\tau})}{1+\cosh (\tilde{\tau})}>\left(\frac{\left|r_{\star}^{\prime}\right|}{1-3 \dot{\Theta}_{\star}}\right) .
$$

Therefore $\omega=\Theta \tilde{\omega}$ never vanishes not even at the conformal boundary.

\subsection{Conformal Gaussian coordinates in the extremal Schwarzschild-de Sitter spacetime}

In this Section we show how the congruence of conformal geodesics with $\gamma=1$ can be used to construct a system of conformal Gaussian coordinates. In view of the periodicity of the maximal extension of the spacetime, the analysis will be restricted to the two triangles shown in Figure 7.

In what follows denote by $\mathrm{eSdS}$ the region in the conformal representation of the extremal Schwarzschild de Sitter spacetime defined by the conformal factor $\Theta$ associated to the congruence of conformal geodesics given by Figure 7. On eSdS consider a spacelike hypersurface $\tilde{\mathcal{S}}$ defined by the condition $r=r_{\star}$. For definiteness let $r_{\star}<r_{\mathcal{H}}$ so that the hypersurface is below the horizon. We use the restriction of the retarded null coordinate $u$ on $\tilde{\mathcal{S}}$ to parametrise points on $\tilde{\mathcal{S}}$-in a slight abuse of notation we denote this restriction by $u_{\star}$; observe that $u_{\star} \in(-\infty, \infty)$. Within eSdS we distinguish two subregions: $\operatorname{eSdS}_{I}$ lying towards the future of $\tilde{\mathcal{S}}$ and $\operatorname{eSdS}_{I I}$ lying towards the past.

The region $\operatorname{eSdS}_{I}$. For $r \in\left[r_{\star}, \infty\right)$ and $u \in(-\infty, \infty)$ let $z \equiv 1 / r$ and $w \equiv \tanh u$. In terms of these coordinates one has

$$
\operatorname{eSdS}_{I}=\left\{z \in\left[0, z_{\star}\right], w \in[-1,1]\right\} .
$$

The analysis in the previous Sections then shows that the map

$$
(w, z):\left[0, \tau_{+}\right] \times(-\infty, \infty) \longrightarrow(-1,1) \times\left[0, z_{\star}\right]
$$

with

$$
w=\tanh u\left(\tau, u_{\star}\right), \quad z=1 / r\left(\tau, u_{\star}\right),
$$


as defined by the solutions to the conformal geodesic equations depends analytically on its parameters. Further the analysis of the conformal deviation equation shows that the Jacobian of the transformation is non-zero for the given range of parameters. Thus, it follows that the inverse map

$$
\left(\tau, u_{\star}\right):(-1,1) \times\left[0, z_{\star}\right] \longrightarrow\left[0, \tau_{+}\right] \times(-\infty, \infty)
$$

with

$$
\tau=\tau(\operatorname{arctanh} w, 1 / z), \quad u_{\star}=u_{\star}(\operatorname{arctanh} w, 1 / z),
$$

is well defined and also an analytic function of its parameters. Thus, ignoring angular coordinates, this inverse map defines a conformal Gaussian system of coordinates in $\mathrm{eSdS}_{I}$. In particular, given any point in $\mathrm{eSdS}_{I}$, there is a unique conformal geodesic passing through it. Thus, the congruence of conformal geodesics covers the whole of eSdS .

The region $\mathbf{e S d S}_{I I}$. In terms of the coordinates $(w, r)$, the region $\operatorname{eSdS}_{I I}$ is described by

$$
\operatorname{eSdS}_{I I}=\left\{w \in(-1,1), r \in\left(0, r_{\star}\right]\right\} .
$$

Again, the analysis carried out in the previous Sections shows that the map

$$
(w, r):\left(\tau_{\downarrow}, 0\right] \times(-1,1) \longrightarrow(-1,1) \times\left(0, r_{\star}\right]
$$

is an analytic function of its parameters. Moreover, the analysis of the conformal geodesic deviation equation shows that it is invertible. Thus, the inverse map

$$
\left(\tau, u_{\star}\right):(-1,1) \times\left(0, r_{\star}\right] \longrightarrow\left(\tau_{\text {夕夕 }}, 0\right] \times(-1,1)
$$

is well-defined and an analytic function of its parameters. Thus, again ignoring angular coordinates, the inverse map defines a conformal Gaussian system of coordinates in $\operatorname{eSdS}_{I I}$. In particular, the congruence of conformal geodesics covers the whole of $\mathrm{eSdS}_{I I}$.

Remark 20. Observe that the parallel horizons bounding the region eSdS are not covered by the congruence of timelike conformal geodesics.

\subsection{Summary of the analysis}

The analysis of the previous Sections can be summarised in the following proposition:

Proposition 1 (Conformal geodesics in the extremal Schwarzschild-de Sitter spacetime). The portion of the extremal Schwarzschild-de Sitter spacetime corresponding to the region eSdS can be covered by a non-singular congruence of conformal geodesics emanating from the singularity at $r=0$ and escaping to the conformal boundary. This congruence can be used to construct a global system of conformal Gaussian coordinates in the spacetime.

\section{The Schwarzschild-anti de Sitter spacetime}

Consistent with the discussion of Section 2.1, for the Schwarzschild-anti de Sitter spacetime $\lambda<0$. The latter will be assumed throughout this Section. In this case one expects to be able to construct a congruence of conformal geodesics that combines the properties of congruences in the Schwarzschild and anti de Sitter spacetime.

\subsection{Basic setup}

In this Section we provide the initial data for the congruence of conformal geodesics in the Schwarzschild-anti de Sitter spacetime and analyse some of its basic properties. 


\subsubsection{Initial data}

As in the case of the subextremal Schwarzschild-de Sitter solution, we set initial data for the congruence of conformal geodesics on the time symmetric hypersurface $\tilde{\mathcal{S}} \equiv\{t=0\}$ of the Schwarzschild-anti de Sitter spacetime. One requires the congruence to be orthogonal to $\tilde{\mathcal{S}}$, consequently, we set

$$
t_{\star}=0, \quad t_{\star}^{\prime}=\frac{1}{\sqrt{D_{\star}}}, \quad r_{\star}^{\prime}=0, \quad r_{\star}>r_{b}
$$

where now $r_{b}$ is given by equation 13 . To find a suitable initial conformal factor and the value of $\beta$ we first look at the limiting case $M=0$. In this case the line element (3) reduces to

$$
\tilde{\boldsymbol{g}}_{a d S}=\left(1+r^{2}\right) \mathbf{d} t \otimes \mathbf{d} t-\left(1+r^{2}\right)^{-1} \mathbf{d} r \otimes \mathbf{d} r-r^{2} \boldsymbol{\sigma} .
$$

Using the the coordinate transformation $r=\sinh \varrho$ the above line element of the anti de Sitter spacetime can be brought to the more standard form

$$
\tilde{\boldsymbol{g}}_{a d S}=\cosh ^{2} \varrho \mathbf{d} t \otimes \mathbf{d} t-\mathbf{d} \varrho \otimes \mathbf{d} \varrho-\sinh ^{2} \varrho \boldsymbol{\sigma} .
$$

It is well known the de Sitter spacetime is conformal to the static Einstein Universe $\left(\mathbb{R} \times \mathbb{S}^{3}, \boldsymbol{g}_{\mathcal{E}}\right)$. The conformal factor realising this conformal embedding is given by

$$
\Xi=\frac{1}{\cosh \varrho}=\frac{1}{\sqrt{1+r^{2}}} .
$$

To see this more clearly we introduce a coordinate $\chi$ via $\tan (\chi / 2)=\tanh (\rho / 2)$. Then a computation shows that $\boldsymbol{g}_{\mathcal{E}}=\Xi^{2} \tilde{\boldsymbol{g}}_{a d S}$ is given by

$$
\boldsymbol{g}_{\mathcal{E}}=\mathbf{d} t \otimes \mathbf{d} t-\mathbf{d} \chi \otimes \mathbf{d} \chi-\sin ^{2} \chi \boldsymbol{\sigma} .
$$

Conformal geodesics for the anti-de Sitter spacetime have been studied in [4 - see also [17] for a discussion of conformal geodesics in the Minkowski, de-Sitter and anti de-Sitter spacetimes. Returning to the $M \neq 0$ case, we will use the conformal factor given in equation (117) to fix the initial data for $\boldsymbol{\beta}$. A calculation readily gives that

$$
\Xi^{-1} \mathbf{d} \Xi=-\frac{r}{1+r^{2}} \mathbf{d} r
$$

The above 1-form suggests setting the initial data

$$
\Theta_{\star}=\frac{1}{\sqrt{1+r_{*}^{2}}}, \quad \boldsymbol{\beta}_{\star}=\tilde{\boldsymbol{\beta}}_{\star}=-\frac{r_{*}}{1+r_{*}^{2}} \mathbf{d} r_{*}
$$

so that

$$
\beta=\frac{r_{\star}}{1+r_{\star}^{2}} \sqrt{1-\frac{M}{r_{\star}}+r_{\star}^{2}}=\frac{\sqrt{r_{*}} \sqrt{r_{*}\left(r_{*}^{2}+1\right)-r_{b}\left(r_{b}^{2}+1\right)}}{r_{*}^{2}+1} .
$$

Notice that according to equation (40) the value of $\gamma$ is fixed by the choice of initial data and it turns out to be

$$
\gamma=\frac{\sqrt{r_{*}\left(r_{*}^{2}+1\right)-r_{b}\left(r_{b}^{2}+1\right)}}{\sqrt{r_{*}}\left(r_{*}^{2}+1\right)} .
$$

Hence, one has

$$
\beta=r_{*} \gamma .
$$

Furthermore, using the constraints 20 one can readily compute the values of $\dot{\Theta}_{\star}$ and $\ddot{\Theta}_{\star}$. One has that

$$
\dot{\Theta}_{\star}=0, \quad \Theta_{\star} \ddot{\Theta}_{\star}=\frac{1}{2}\left(1+\tilde{\boldsymbol{g}}^{\sharp}\left(\boldsymbol{\beta}_{\star}, \boldsymbol{\beta}_{\star}\right)\right)=\frac{1+M r+r^{2}}{2\left(1+r^{2}\right)^{2}}>0 .
$$


It follows from the above that

$$
\Theta=\Theta_{\star}+\frac{1}{2} \ddot{\Theta}_{\star} \tau^{2}
$$

cannot vanish for any value of $\tau$ if $r \in\left[r_{b}, \infty\right)$. Accordingly, the conformal geodesics associated to this conformal factor do not intersect the conformal boundary unless they are initially tangent to it. Using the above conformal factor one can compute the the explicit relation between $\tau$ and $\tilde{\tau}$ using (21). One finds that

$$
\tilde{\tau}=\frac{2\left(r_{\star}^{2}+1\right)}{\sqrt{M r_{\star}+r_{\star}^{2}+1}} \arctan \left(\frac{\tau \sqrt{r_{\star}\left(M+r_{\star}\right)+1}}{2 \sqrt{r_{\star}^{2}+1}}\right) .
$$

Remark 21. Using formula 121 one can readily verify that for finite values of $r_{\star}$

$$
\tilde{\tau} \rightarrow \frac{\pi\left(r_{\star}+1\right)}{\sqrt{r_{\star}^{2}+M r_{\star}+1}} \quad \text { as } \quad \tau \rightarrow \infty .
$$

Moreover, taking the double limit

$$
\tilde{\tau} \rightarrow \pi \quad \text { as } \quad r_{\star} \rightarrow \infty, \quad \tau \rightarrow \infty .
$$

This is a manifestation of a phenomenon already observed in the anti de Sitter spacetime in which the $g$-proper time only covers a finite portion of the temporal extent of the Einstein cylinder - see [4. In order to continue the description of a conformal geodesic with the $g$-proper time one needs to perform a reparametrisation by means of a Möbius transformation — see e.g. [17.

\subsubsection{Technical observations}

With the choice of $\beta$ given by the positive square root of equation 118 , the solution of equation (41) can be written as

$$
\tilde{\tau}=\left(1+r_{\star}{ }^{2}\right) \int_{r}^{r_{\star}} \sqrt{\frac{\bar{r} r_{\star}}{\left(r_{\star}-\bar{r}\right) P\left(\bar{r}, r_{\star}, M\right)}} \mathrm{d} \bar{r}, \quad r_{\star} \geq r_{b},
$$

where

$$
\begin{aligned}
P\left(r, r_{\star}, M\right) & \equiv r_{\star} r^{2}\left(M r_{\star}+r_{\star}^{2}+1\right)+r_{\star} r\left(M\left(r_{\star}{ }^{2}+2\right)-r_{\star}\left(r_{\star}{ }^{2}+1\right)\right)+M\left(r_{\star}{ }^{2}+1\right)^{2} \\
& =\left(M r_{\star}+r_{\star}{ }^{2}+1\right)\left(r-\alpha_{+}\left(M, r_{\star}\right)\right)\left(r-\alpha_{-}\left(M, r_{\star}\right)\right) .
\end{aligned}
$$

Since $M>0$, if $\alpha_{ \pm}\left(M, r_{\star}\right)$ are complex then $P\left(r, r_{\star}, M\right)>0$. On the other hand, if $\alpha_{ \pm}\left(M, r_{\star}\right)$ are real then

$$
\alpha_{+}\left(M, r_{\star}\right)>\alpha_{-}\left(M, r_{\star}\right) .
$$

Moreover, if $\alpha_{ \pm}\left(M, r_{\star}\right)$ are real, then we have the following result:

Lemma 3. If $M>0$ and $\alpha_{ \pm}\left(M, r_{\star}\right)$ are real then

$$
\alpha_{+}\left(M, r_{\star}\right)<r_{\star}
$$

Proof. One has the following explicit relations

$$
\begin{aligned}
& \alpha_{ \pm}\left(M, r_{\star}\right) \equiv \frac{r_{\star}\left(-M\left(r_{\star}^{2}+2\right)+r_{\star}\left(r_{\star}^{2}+1\right)\right) \pm \sqrt{\Delta\left(M, r_{\star}\right)}}{2 r_{\star}\left(M r_{\star}+r_{\star}^{2}+1\right)}, \\
& \Delta\left(M, r_{\star}\right) \equiv-M^{2}\left(3 r_{\star}^{2}+4\right) r_{\star}^{4}-2 M\left(3 r_{\star}^{6}+9 r_{\star}^{4}+8 r_{\star}^{2}+2\right) r_{\star}+\left(r_{\star}^{2}+1\right)^{2} r_{\star}^{4} .
\end{aligned}
$$

We now notice that

$$
0 \leq 4 M r_{\star}\left(3 r_{\star}^{4}+4 r_{\star}^{2}+1\right)\left(M r_{\star}+r_{\star}^{2}+1\right)
$$


which holds because as $M>0$ and $r_{\star}>0$ all the factors are positive. Expanding out the product we find that the inequality (124) can be rewritten in the form

$$
\Delta\left(M, r_{\star}\right) \leq\left(3 M r_{\star}^{3}+2 M r_{\star}+r_{\star}^{4}+r_{\star}^{2}\right)^{2} .
$$

Under the assumptions that $\alpha_{ \pm}$are real one has that $\Delta\left(M, r_{\star}\right) \geq 0$, so that

$$
\sqrt{\Delta\left(M, r_{\star}\right)} \leq\left|3 M r_{\star}^{3}+2 M r_{\star}+r_{\star}^{4}+r_{\star}^{2}\right|=3 M r_{\star}^{3}+2 M r_{\star}+r_{\star}^{4}+r_{\star}^{2} .
$$

Next use the identity

$$
3 M r_{\star}^{3}+2 M r_{\star}+r_{\star}^{4}+r_{\star}^{2}=2 r_{\star}^{2}\left(1+M r_{\star}+r_{\star}^{2}\right)-\left(r_{\star}^{2}+r_{\star}^{4}-M r_{\star}\left(2+r_{\star}^{2}\right)\right),
$$

which enables us to write the inequality 125 in the form

$$
\left(r_{\star}^{2}+r_{\star}^{4}-M r_{\star}\left(2+r_{\star}^{2}\right)\right)+\sqrt{\Delta\left(M, r_{\star}\right)} \leq 2 r_{\star}^{2}\left(1+M r_{\star}+r_{\star}^{2}\right) .
$$

Finally, as $r_{\star}\left(1+M r_{\star}+r_{\star}^{2}\right) \geq 0$ we conclude that $\alpha_{+}\left(M, r_{\star}\right)<r_{\star}$.

Lemma 4. If $\left(r_{\star}-r\right) P\left(r, r_{\star}, M\right)>0$ then $r<r_{*}$ and $P\left(r, r_{\star}, M\right)>0$.

Proof. If $\left(r_{\star}-r\right) P\left(r, r_{\star}, M\right)>0$ then either

$$
\text { (a) } \quad r_{\star}>r \quad \text { and } \quad P\left(r, r_{\star}, M\right)>0
$$

or

$$
\text { (b) } \quad r_{\star}<r \quad \text { and } \quad P\left(r, r_{\star}, M\right)<0 .
$$

If $\alpha_{ \pm}\left(M, r_{\star}\right)$ are complex then $P\left(r, r_{\star}, M\right)>0$ and case (b) cannot hold so the lemma is proven. If $\alpha_{ \pm}\left(M, r_{\star}\right)$ are real then by virtue of Lemma 3 one has $\alpha_{-}\left(M, r_{\star}\right)<\alpha_{+}\left(M, r_{\star}\right)<r_{\star}$. If case (b) holds then $P\left(r, r_{\star}, M\right)<0$ which, in turn, can only be true if $\left(r-\alpha_{+}\left(M, r_{\star}\right)\right)\left(r-\alpha_{-}\left(M, r_{\star}\right)\right)<0$. Nevertheless, by Lemma $3, r>r_{\star}>\alpha_{+}\left(M, r_{\star}\right)$ and, consequently, since $P\left(r, r_{\star}, M\right)<0$ then $r<\alpha_{-}\left(M, r_{\star}\right)$. However, this is a contradiction as $\alpha_{-}\left(M, r_{\star}\right)<\alpha_{+}\left(M, r_{\star}\right)$. Therefore, case (b) cannot hold which then proves the lemma.

Remark 22. Notice that Lemma 4 implies that if the radicand in the right hand side of equation (122) is positive then necessarily $r<r_{\star}$. Consequently, $r(\tilde{\tau})$ is decreasing if $\tilde{\tau} \neq 0$. By continuity, $r(\tilde{\tau})$ is decreasing until it reaches a value of $\tilde{\tau}$ where $r^{\prime}(\tilde{\tau})$ vanishes. Using equation (41) one has that $r^{\prime}(\tilde{\tau})$ vanishes whenever $P\left(r, r_{\star}, M\right)$ vanishes. In other words, if $\alpha_{ \pm}\left(M, r_{\star}\right)$ are real then $r^{\prime}(\tilde{\tau})$ vanishes at $\tilde{\tau}_{\alpha_{ \pm}}$where $r=\alpha_{ \pm}\left(M, r_{\star}\right)$ and at $\tilde{\tau}=0$ where $r=r_{\star}$. If $\alpha_{ \pm}\left(M, r_{\star}\right)$ are complex then $r^{\prime}(\tilde{\tau})$ is non-zero for $\tilde{\tau}>0$ so $r(\tilde{\tau})$ is always decreasing.

Remark 23. In the sequel, to simplify the notation $\alpha_{ \pm}\left(M, r_{\star}\right)$ will be simply denoted by $\alpha_{ \pm}$. Moreover, for future reference notice that equation 122 can be written as

$$
\tilde{\tau}=\frac{\left(1+r_{\star}^{2}\right) \sqrt{r_{\star}}}{\sqrt{M r_{\star}+r_{\star}^{2}+1}} \int_{r}^{r_{\star}} \sqrt{\frac{\bar{r}}{\left(r_{\star}-\bar{r}\right)\left(\bar{r}-\alpha_{+}\right)\left(\bar{r}-\alpha_{-}\right)}} d \bar{r} .
$$

Following the above discussion, depending on the sign of $\Delta\left(M, r_{\star}\right)$, we may distinguish three possibilities which are discussed.

\subsection{Qualitative analysis of the behaviour of the curves}

In this Section we analyse the different qualitative behaviours of the conformal geodesics defined by the initial conditions given in the previous Section. There are, broadly, three types of geodesics: a set of geodesics parallel to the conformal boundary, geodesics reaching timelike infinity and finally geodesics falling into the singularity. 


\subsubsection{Conformal geodesics entering the horizon}

Consider $r_{\star}$ such that $\Delta\left(M, r_{\star}\right)<0$. In this case $\alpha_{ \pm}$are complex and $P\left(r, r_{\star}, M\right)$ is strictly positive. Therefore, there are no turning points and $0<r<r_{\star}$. The conformal geodesics get through the event horizon $r=r_{b}$ and end up in the singularity $r=0$ at

$$
\tilde{\tau}_{\text {夕 }} \equiv\left(1+r_{\star}^{2}\right) \int_{0}^{r_{\star}} \sqrt{\frac{r r_{\star}}{\left(r_{\star}-r\right) P\left(r, r_{\star}, M\right)}} \mathrm{d} r .
$$

To verify that $\tilde{\tau}_{\not{b}}$ is finite observe that since $P\left(r, r_{\star}, M\right)$ is strictly positive then there exist a small $\delta>0$ such that $P\left(r, r_{\star}, M\right) \geq \delta$. Consequently

$$
\tilde{\tau}_{\text {夕夕 }} \leq\left(1+r_{\star}^{2}\right) \sqrt{\frac{r_{\star}}{\delta}} \int_{0}^{r_{\star}} \sqrt{\frac{r}{\left(r_{\star}-r\right)}} \mathrm{d} r=\left(1+r_{\star}^{2}\right) \sqrt{\frac{r_{\star}}{\delta}} \frac{\pi}{2} r_{\star}<\infty .
$$

Explicit expressions in terms of elliptic functions. In the case $\Delta\left(M, r_{\star}\right)<0$ one has

$$
0<r<r_{\star} \quad \text { and } \quad \bar{\alpha}_{+}=\alpha_{-} \in \mathbb{C} .
$$

One can use this information to rewrite the integral given in equation 126 in terms of elliptic functions. In particular, using formulae 259.07 and 361.62 of [2] with $R(t)=a-t$ and parameters

$$
a=0, \quad b=r_{\star}, \quad c=\alpha_{+}, \quad \bar{c}=\alpha_{-},
$$

one obtains

$$
\tilde{\tau}=\frac{\left(1+r_{\star}^{2}\right) \sqrt{r_{\star}}}{\sqrt{M r_{\star}+r_{\star}^{2}+1}}\left(\frac{A r_{\star}}{A-B}\right)\left(u-\frac{1}{1+\alpha}\left(\Pi\left(\varphi, \frac{\alpha^{2}}{\alpha^{2}-1}, k\right)-\alpha f_{1}\right)\right)
$$

where $\operatorname{dn} u, \operatorname{sn} u$ and $\operatorname{cn} u$ denote the delta amplitude, the sine amplitude and cosine amplitude functions (Jacobi elliptic functions). The function $\operatorname{sd} u$ is defined as $\operatorname{sd} u \equiv \operatorname{sn} u / \operatorname{dn} u$ and $\Pi\left[\phi, \alpha^{2}, \kappa\right]$ is the incomplete elliptic integral of the third kind. The constants $A, B, g, k$ and $k^{\prime}$ are determined in terms of $r_{\star}$ and $\alpha_{+}$via

$$
\begin{gathered}
A \equiv \frac{1}{2}\left(\operatorname{Re}\left(\alpha_{+}\right)^{2}-\operatorname{Im}\left(\alpha_{+}\right)^{2}\right), \quad B \equiv\left(r_{\star}-\operatorname{Re}\left(\alpha_{+}\right)\right)^{2}-\frac{1}{2} \operatorname{Im}\left(\alpha_{+}\right)^{2}, \\
\alpha \equiv \frac{A-B}{A+B}, \quad g \equiv \frac{1}{\sqrt{A B}}, \quad k^{2} \equiv \frac{r_{\star}^{2}-(A-B)^{2}}{4 A B}, \quad k^{\prime} \equiv \sqrt{1-k^{2}}, \\
\operatorname{cn} u=\cos \varphi, \quad \varphi=\cos ^{-1}\left(\frac{B r+A\left(r-r_{\star}\right)}{B r-A\left(r-r_{\star}\right)}\right), \\
f_{1}= \begin{cases}\sqrt{\frac{1-\alpha^{2}}{k^{2}+k^{\prime 2} \alpha^{2}}} \arctan \left(\frac{k^{2}+k^{\prime 2} \alpha}{1-\alpha^{2}} \operatorname{sd} u\right) & \text { if } \frac{\alpha^{2}}{\alpha^{2}-1}<k^{2}, \\
\operatorname{sd} u & \text { if } \frac{\alpha^{2}}{\alpha^{2}-1}=k^{2}, \\
\frac{1}{2} \sqrt{\frac{1-\alpha^{2}}{k^{2}+k^{\prime 2} \alpha^{2}}} \ln \left(\frac{\operatorname{dn} u \sqrt{k^{2}+k^{\prime 2} \alpha^{2}}+\operatorname{sn} u \sqrt{\alpha^{2}-1}}{\operatorname{dn} u \sqrt{k^{2}+k^{\prime 2} \alpha^{2}}-\operatorname{sn} u \sqrt{\alpha^{2}-1}}\right) & \text { in }\end{cases}
\end{gathered}
$$

Remark 24. The expression for $\tilde{\tau}$ given by $(128)$ can be seen to be an analytic function of its arguments.

\subsubsection{Critical conformal geodesic}

We consider next $r_{\circledast}$ such that $\Delta\left(M, r_{\circledast}\right)=0$. In this case $P\left(r, r_{\circledast}, M\right)$ has a double root and the integral expression (122) takes the form

$$
\tilde{\tau}=\frac{1+r_{\circledast}^{2}}{\sqrt{M r_{\circledast}+r_{\circledast}^{2}+1}} \int_{r}^{r_{\circledast}} \sqrt{\frac{\bar{r} r_{\circledast}}{\left(r_{\circledast}-\bar{r}\right)\left(\bar{r}-\alpha\left(M, r_{\circledast}\right)\right)^{2}}} \mathrm{~d} \bar{r},
$$


with

$$
\alpha\left(M, r_{\circledast}\right)=\frac{r_{\circledast}^{3}+r_{\circledast}-M\left(r_{\circledast}^{2}+2\right)}{2\left(M r_{\circledast}+r_{\circledast}^{2}+1\right)} .
$$

In fact, in this particular case one can compute the integral in terms of elementary functions, the result being

$$
\begin{aligned}
& \tilde{\tau}=\left(1+r_{\circledast}^{2}\right) \sqrt{\frac{r_{\circledast}}{M r_{\circledast}+r_{\circledast}^{2}+1}} \times \\
& \left(2 \sqrt{\frac{\alpha\left(M, r_{\circledast}\right)}{r_{\circledast}-\alpha\left(M, r_{\circledast}\right)}} \log \left|\frac{\left(\frac{r}{r_{\circledast}-r}\right)^{\frac{1}{2}}+\left(\frac{\alpha\left(M, r_{\circledast}\right)}{r_{\circledast}-\alpha\left(M, r_{\circledast}\right)}\right)^{\frac{1}{2}}}{\left(\frac{r}{r_{\circledast}-r}\right)^{\frac{1}{2}}-\left(\frac{\alpha\left(M, r_{\circledast}\right)}{r_{\circledast}-\alpha\left(M, r_{\circledast}\right)}\right)^{\frac{1}{2}}}\right|+\pi-2 \arctan \left(\frac{r}{r_{\circledast}-r}\right)^{\frac{1}{2}}\right)
\end{aligned}
$$

where the integral is carried out using that $\alpha\left(M, r_{\circledast}\right)<r_{\circledast}$ and $r<r_{\circledast}$ which arise respectively from Lemmas 3 and 4 . Observe that

$$
\lim _{r \rightarrow r_{\circledast}^{-}} \tilde{\tau}=0, \quad \lim _{r \rightarrow \alpha\left(M, r_{\circledast}\right)^{+}} \tilde{\tau}=\infty .
$$

Hence we conclude that $r$ in equation (129) has to be taken such that $\alpha\left(M, r_{\circledast}\right)<r<r_{\circledast}$.

Assumption 1. For the subsequent discussion the location of $\alpha\left(M, r_{\circledast}\right)$ relative to $r_{b}$ is required. Numerical evaluations suggest that $r_{b}<\alpha\left(M, r_{\circledast}\right)$. In the following $r_{b}<\alpha\left(M, r_{\circledast}\right)$ will be assumed.

Notice that $r_{b}<\alpha\left(M, r_{\circledast}\right)$ implies that this geodesic never enters into the black hole region. Hence the conformal geodesic starting at $r=r_{\circledast}$ with $r_{\circledast}$ satisfying the condition $\Delta\left(M, r_{\circledast}\right)=0$ separates the conformal geodesics which go to the black hole region and end up in the singularity from those which do not enter this region. This conformal geodesic is depicted in Figure 9.

Remark 25. It can be readily be verified that the expression for $\tilde{\tau}$ is an analytic expression of its parameters except at $r=\alpha\left(M, r_{\circledast}\right)$.

To further analyse the properties of the critical conformal geodesic we need to study the behaviour of $t\left(r, r_{\circledast}\right)$ as well. This analysis is carried out in the remainder of this Section. Using the chain rule and equations 41 and 42 we get

$$
\frac{\mathrm{d} r}{\mathrm{~d} t}=-\frac{D(r)}{|\gamma+\beta r|} \sqrt{(\gamma+\beta r)^{2}-D(r)} .
$$

Replacing of $\beta, \gamma$ and $D(r)$ for the Schwarzschild-anti de Sitter case, a computation renders

$$
t\left(r, r_{\circledast}\right)=\frac{\gamma\left(1+r_{\circledast}^{2}\right)}{\sqrt{1+r_{\circledast}\left(r_{\circledast}+r_{b}\left(1+r_{b}^{2}\right)\right)}} \int_{r}^{r_{\circledast}} \frac{\bar{r}\left(1+r_{\circledast} \bar{r}\right)}{\left(\bar{r}-r_{b}\right)\left(1+\bar{r}^{2}+\bar{r} r_{b}+r_{b}^{2}\right)}\left[\frac{\bar{r}}{\left(r_{\circledast}-\bar{r}\right)\left(\bar{r}-\alpha\left(M, r_{\circledast}\right)\right)^{2}}\right]^{\frac{1}{2}} \mathrm{~d} \bar{r} .
$$

We use now the following inequalities

$$
\frac{r\left(1+r r_{\circledast}\right)}{\left(r-r_{b}\right)\left(1+r^{2}+r r_{b}+r_{b}^{2}\right)}>\frac{r_{b}}{3\left(r_{\circledast}-r_{b}\right)}, \quad \frac{r}{\left(r_{\circledast}-r\right)\left(r-\alpha\left(M, r_{\circledast}\right)\right)^{2}}>\frac{r_{b}}{\left(r_{\circledast}-r_{b}\right)\left(r-\alpha\left(M, r_{\circledast}\right)\right)^{2}},
$$

which are valid for all values of $r$ such that $\alpha\left(M, r_{\circledast}\right)<r<r_{\circledast}$. From these we get

$$
\begin{aligned}
& \int_{r}^{r_{\circledast}} \frac{\bar{r}\left(1+r_{\circledast} \bar{r}\right)}{\left(\bar{r}-r_{b}\right)\left(1+\bar{r}^{2}+\bar{r} r_{b}+r_{b}^{2}\right)}\left[\frac{\bar{r}}{\left(r_{\circledast}-\bar{r}\right)\left(\bar{r}-\alpha\left(M, r_{\circledast}\right)\right)^{2}}\right]^{\frac{1}{2}} \mathrm{~d} \bar{r}> \\
& \int_{r}^{r_{\circledast}}\left(\frac{r_{b}}{r_{\circledast}-r_{b}}\right)^{\frac{3}{2}} \frac{\mathrm{d} \bar{r}}{3\left(\bar{r}-\alpha\left(M, r_{\circledast}\right)\right)}=\frac{1}{3}\left(\frac{r_{b}}{r_{\circledast}-r_{b}}\right)^{\frac{3}{2}} \log \left(\frac{r_{\circledast}-\alpha\left(M, r_{\circledast}\right)}{r-\alpha\left(M, r_{\circledast}\right)}\right) .
\end{aligned}
$$


Hence

$$
\lim _{r \rightarrow \alpha\left(M, r_{\circledast}\right)^{+}} t\left(r, r_{\circledast}\right)>\lim _{r \rightarrow \alpha\left(M, r_{\circledast}\right)^{+}} \frac{1}{3}\left(\frac{r_{b}}{r_{\circledast}-r_{b}}\right)^{\frac{3}{2}} \log \left(\frac{r_{\circledast}-\alpha\left(M, r_{\circledast}\right)}{r-\alpha\left(M, r_{\circledast}\right)}\right)=\infty .
$$

This shows that the critical conformal geodesic reaches infinite coordinate time but neither enters the black hole nor escapes to infinity. Therefore it asymptotes to a region which is neither conformal infinity nor the singularity.

\subsubsection{Conformal geodesics not entering the horizon}

Finally, consider values of $r_{\star}$ for which $\Delta\left(M, r_{\star}\right)>0$. In this case the roots $\alpha_{ \pm}$of the polynomial $P\left(r, r_{\star}, M\right)$ are real and Lemma 3 applies. This implies that $r_{\star}>r>\alpha_{+}$and the limit

$$
\tilde{\tau}\left(\alpha_{+}\right) \equiv\left(1+r_{\star}^{2}\right) \lim _{r \rightarrow \alpha_{+}} \int_{r}^{r_{\star}} \sqrt{\frac{\bar{r} r_{\star}}{\left(r_{\star}-\bar{r}\right) P\left(\bar{r}, r_{\star}, M\right)}} \mathrm{d} \bar{r} .
$$

is finite. To see this, recall that equation 126 implies that the latter limit can be computed via

$$
\lim _{r \rightarrow \alpha_{+}} \int_{r}^{r_{\star}} \sqrt{\frac{\bar{r}}{\left(r_{\star}-\bar{r}\right)\left(\bar{r}-\alpha_{+}\right)\left(\bar{r}-\alpha_{-}\right)}} \mathrm{d} \bar{r} .
$$

Fix a constant value $R$ with $\alpha_{+}<r<R<r_{\star}$. Under these conditions we have the inequalities

$$
\frac{\bar{r} r_{\star}}{r_{\star}-\bar{r}}<\frac{R r_{\star}}{r_{\star}-R}, \quad \frac{1}{\bar{r}-\alpha_{-}}<\frac{1}{\alpha_{+}-\alpha_{-}},
$$

from which

$$
\begin{array}{r}
\int_{r}^{r_{\star}} \sqrt{\frac{\bar{r}}{\left(r_{\star}-\bar{r}\right)\left(\bar{r}-\alpha_{+}\right)\left(\bar{r}-\alpha_{-}\right)}} \mathrm{d} \bar{r}<\left(\frac{R r_{\star}}{\left(r_{\star}-R\right)\left(\alpha_{+}-\alpha_{-}\right)}\right)^{\frac{1}{2}} \int_{r}^{r_{\star}} \frac{\mathrm{d} \bar{r}}{\sqrt{\bar{r}-\alpha_{+}}} \\
=2\left(\frac{R r_{\star}}{\left(r_{\star}-R\right)\left(\alpha_{+}-\alpha_{-}\right)}\right)^{\frac{1}{2}} \sqrt{r_{\star}-\alpha_{+}}, \quad \alpha_{+}<r<R<r_{\star} .
\end{array}
$$

Hence,

$$
\lim _{r \rightarrow \alpha_{+}} \int_{r}^{r_{\star}} \sqrt{\frac{\bar{r}}{\left(r_{\star}-\bar{r}\right)\left(\bar{r}-\alpha_{+}\right)\left(\bar{r}-\alpha_{-}\right)}} \mathrm{d} \bar{r}<2\left(\frac{R r_{\star}}{\left(r_{\star}-R\right)\left(\alpha_{+}-\alpha_{-}\right)}\right)^{\frac{1}{2}} \sqrt{r_{\star}-\alpha_{+}} .
$$

Thus, the value $r=\alpha_{+}$is reached in a finite amount of physical proper time. Now, it can be readily verified that $\mathrm{d} r / \mathrm{d} \tilde{\tau}=0$ at $r=\alpha_{+}$. Thus, at $r=\alpha_{+}$one has a turning point. The conformal geodesic reaching this point can be smoothly extended by means of a reflection of the conformal geodesic with respect to the horizontal line defined by $\tilde{\tau}=\tilde{\tau}\left(\alpha_{+}\right)$. By repeating this procedure an infinite number of times one gets an inextendible curve which is a periodic function in the variable $\tilde{\tau}$ when represented in the form $r=r\left(\tilde{\tau}, r_{\star}\right)$-see Figure 9. The period is given by $2 \alpha_{+}\left(M, r_{\star}\right)$. In particular, one has that, although the value of $r(\tilde{\tau})$ remains bounded for $\tilde{\tau} \in[0, \infty)$, it does not have a limit as $\tilde{\tau} \rightarrow \infty$. Moreover, making use of expression for the coordinate $t$, one can readily verify that two consecutive turning points are reached in a finite amount of coordinate time $t$. Also, an explicit computation shows that

$$
\lim _{r_{\star} \rightarrow \infty} \alpha_{+}\left(M, r_{\star}\right)=\infty
$$

which implies that the conformal geodesics approach a vertical line in the limit $r_{\star} \rightarrow \infty-$ the timelike conformal boundary.

Remark 26. The late time behaviour of the conformal geodesics close to the conformal boundary in the Schwarzschild-de Sitter spacetime is similar to the behaviour observed in the anti de Sitter spacetime in which the vicinity of the conformal boundary (and, in fact, the whole spacetime) is ruled by conformal geodesics. Our analysis thus shows that there is an infinite number of conformal geodesics between the critical curve and the conformal boundary which neither fall into the black hole nor escape to the conformal boundary. 
Explicit expressions in terms of elliptic functions. In the case $\Delta\left(M, r_{\star}\right)>0$ one has three different subcases depending on the sign on $\alpha_{ \pm}$:
a) $r_{\star}>r>\alpha_{+}>\alpha_{-}>0$,
b) $r_{\star}>r>\alpha_{+}>0>\alpha_{-}$,
c) $r_{\star}>r>0>\alpha_{+}>\alpha_{-}$.

All these cases can be discussed in a unified way using the formulae given in [2]. To do so, let

$$
\begin{array}{r}
\varphi \equiv \sin ^{-1}\left(\sqrt{\frac{(b-d)(a-r)}{(a-b)(r-d)}}\right), \quad \alpha^{2} \equiv \frac{(a-d)(c-d)}{(a-c)(b-d)}, \quad \kappa^{2} \equiv \frac{(a-c)(c-d)}{(a-c)(b-d)}, \\
g \equiv \frac{2}{\sqrt{(a-c)(b-d)}}, \quad s n^{2} u \equiv \frac{(b-d)(a-r)}{(a-b)(r-d)}, \quad s n u_{1} \equiv \sin \varphi \Rightarrow u_{1}=\mathrm{F}(\varphi, \kappa),
\end{array}
$$

where sn denotes the sine amplitude function and $\mathrm{F}(\varphi, \kappa)$ is the incomplete elliptic integral of the first kind. For case a) using formula 257.02 of [2] with parameters

$$
a=r_{\star}, \quad b=\alpha_{+}, \quad c=\alpha_{-}, \quad d=0,
$$

the integral (126) can be expressed as

$$
\tilde{\tau}=\frac{\left(1+r_{\star}^{2}\right) \sqrt{r_{\star}}}{\sqrt{M r_{\star}+r_{\star}^{2}+1}}(a-d) g \Pi\left[\varphi, \alpha^{2}, \kappa\right] .
$$

For case b) using formula 257.13 of [2] with parameters

$$
a=r_{\star}, \quad b=\alpha_{+}, \quad c=0, \quad d=\alpha_{-},
$$

one obtains

$$
\begin{aligned}
\tilde{\tau} & =\left.\frac{\left(1+r_{\star}^{2}\right) \sqrt{r_{\star}}}{\sqrt{M r_{\star}+r_{\star}^{2}+1}} \frac{(c-a) g}{\alpha^{2}}\left(k^{2} u+\left(\alpha^{2}-k^{2}\right) \Pi\left[\varphi, \alpha^{2}, \kappa\right]\right)\right|_{0} ^{u_{1}} \\
& =\frac{\left(1+r_{\star}^{2}\right) \sqrt{r_{\star}}}{\sqrt{M r_{\star}+r_{\star}^{2}+1}} \frac{(c-a) g}{\alpha^{2}}\left(k^{2} u_{1}+\left(\alpha^{2}-k^{2}\right) \Pi\left[\varphi, \alpha^{2}, \kappa\right]\right) .
\end{aligned}
$$

For case c) using formula 257.15 of [2] with parameters

$$
a=r_{\star}, \quad b=0, \quad c=\alpha_{+}, \quad d=\alpha_{-},
$$

one obtains

$$
\begin{aligned}
\tilde{\tau} & =\left.\frac{\left(1+r_{\star}^{2}\right) \sqrt{r_{\star}}}{\sqrt{M r_{\star}+r_{\star}^{2}+1}} \frac{(b-a) g}{\alpha^{2}}\left(u+\left(\alpha^{2}-1\right) \Pi\left[\varphi, \alpha^{2}, \kappa\right]\right)\right|_{0} ^{u_{1}} \\
& =\frac{\left(1+r_{\star}^{2}\right) \sqrt{r_{\star}}}{\sqrt{M r_{\star}+r_{\star}^{2}+1}} \frac{(b-a) g}{\alpha^{2}}\left(u_{1}+\left(\alpha^{2}-1\right) \Pi\left[\varphi, \alpha^{2}, \kappa\right]\right) .
\end{aligned}
$$

In these expressions $\Pi\left[\phi, \alpha^{2}, \kappa\right]$ is the incomplete elliptic integral of the third kind.

\subsubsection{Conformal geodesic starting at the bifurcation sphere}

To study the conformal geodesic which starts at the bifurcation sphere $r_{\star}=r_{b}$ it is necessary that we rewrite the conformal geodesic equations we have developed in Kruskal-like coordinates to cover the maximal extension of the Schwarzschild anti de Sitter spacetime.

To start the discussion notice that for the Schwarzschild-anti de Sitter solution, the EddingtonFinkelstein coordinates defined by equation $(6)$ take the form

$$
\begin{gathered}
u=t+\frac{r_{b} \log \left(\frac{r^{2}+r_{b} r+r_{b}^{2}+1}{\left(r-r_{b}\right)^{2}}\right)}{6 r_{b}^{2}+2}-\frac{\left(3 r_{b}^{2}+2\right) \arctan \left(\frac{2 r+r_{b}}{\sqrt{3 r_{b}^{2}+4}}\right)}{\left(3 r_{b}^{2}+1\right) \sqrt{3 r_{b}^{2}+4}} \\
v=t-\frac{r_{b} \log \left(\frac{r^{2}+r_{b} r+r_{b}^{2}+1}{\left(r-r_{b}\right)^{2}}\right)}{6 r_{b}^{2}+2}+\frac{\left(3 r_{b}^{2}+2\right) \arctan \left(\frac{2 r+r_{b}}{\sqrt{3 r_{b}^{2}+4}}\right)}{\left(3 r_{b}^{2}+1\right) \sqrt{3 r_{b}^{2}+4}} .
\end{gathered}
$$




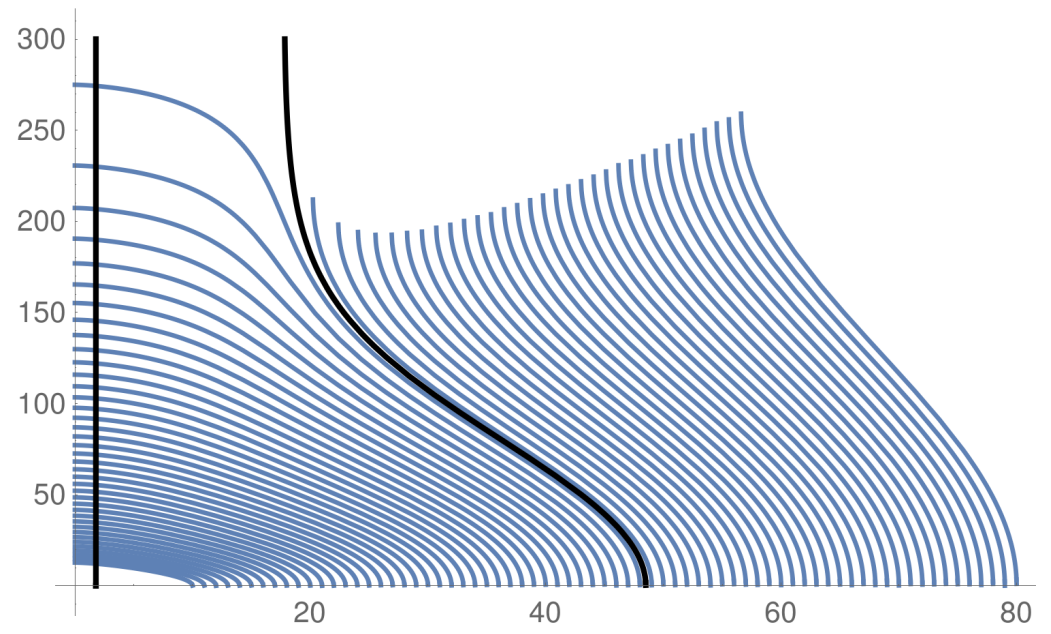

Figure 9: Congruence of conformal geodesics in the exterior region of Schwarzschild anti-de Sitter for $M=7.5$ and $\beta$ given by (118). The horizontal axis corresponds to $r$ and the vertical axis to $\tilde{\tau}$. The thick curve is the separation (critical) geodesic and the thick vertical line corresponds to $r_{b}$. Geodesics on the left to the separation geodesic go towards the singularity whereas geodesics to the right stay away from the singularity.

From these coordinates, one defines the usual Kruskal-like coordinates as follows:

$$
U=\arctan [\exp (\alpha u)], \quad V=\arctan [-\exp (-\alpha v)],
$$

where $\alpha$ is a constant given by

$$
\alpha \equiv-\frac{3 r_{b}^{2}+1}{2 r_{b}}
$$

The radial coordinate can be implicitly written in terms of the Kruskal-like coordinates by means of the relation

$$
\tan (U) \tan (V)=\frac{\left(r_{b}-r\right) \Delta\left(r, r_{b}\right)^{2}}{\sqrt{r^{2}+r r_{b}+r_{b}^{2}+1}}
$$

where

$$
\Delta\left(r, r_{b}\right) \equiv \exp \left(\frac{\left(3 r_{b}^{2}+2\right)}{2 r_{b} \sqrt{3 r_{b}^{2}+4}} \arctan \left(\frac{2 r+r_{b}}{\sqrt{3 r_{b}^{2}+4}}\right)\right) .
$$

Using this relation, we can compute the explicit form of the metric in the Kruskal-like coordinates

$$
\begin{aligned}
& \tilde{\boldsymbol{g}}=G\left(r, r_{b}\right) e^{\alpha(U-V)} \cosh (\alpha U) \cosh (\alpha V)(\mathbf{d} U \otimes \mathbf{d} V+\mathbf{d} V \otimes \mathbf{d} U)-\boldsymbol{\sigma} \\
& G\left(r, r_{b}\right) \equiv \frac{16\left(1+r^{2}+r r_{b}+r_{b}^{2}\right)^{2} r_{b}^{2}}{r\left(1+3 r_{b}\right)^{2}} \exp \left(\frac{-\left(2+3 r_{b}\right)}{\sqrt{4+3 r_{b}^{2}}} \arctan \left(\frac{2 r+r_{b}}{\sqrt{4+3 r_{b}^{2}}}\right)\right) .
\end{aligned}
$$

A computation then shows that the conformal geodesic equations for curves with initial datum $r_{\star}=r_{b}$ are given by

$$
\begin{aligned}
& U^{\prime \prime}=\frac{2\left(U^{\prime}\right)^{2}}{\sin (2 U)\left(3 r_{b}^{2}+1\right)}\left(\left(3 r_{b}^{2}+1\right) \cos (2 U)-\frac{r_{b}\left(2 r^{3}+r_{b}^{3}+r_{b}\right)}{r^{2}}\right) \\
& \begin{aligned}
& r^{\prime \prime}= \frac{4 r_{b}\left(2 r^{3}+r_{b}^{3}+r_{b}\right) U^{\prime}}{\sin ^{2}(2 U)\left(3 r_{b}^{2}+1\right)^{2} r^{3}} \\
& \quad \quad \times\left(\left(3 r_{b}^{2}+1\right) r r^{\prime} \sin (2 U)+2 r_{b}\left(r_{b}-r\right)\left(r\left(r+r_{b}\right)+r_{b}^{2}+1\right) U^{\prime}\right), \\
& 2\left(r_{*}^{2}+1\right)\left(U^{\prime} \frac{\sin (2 V)}{\sin (2 U)}-V^{\prime}\right)\left(2 r_{b} r^{3}+r_{b}^{2}\left(r_{b}^{2}+1\right)\right)=0
\end{aligned}
\end{aligned}
$$




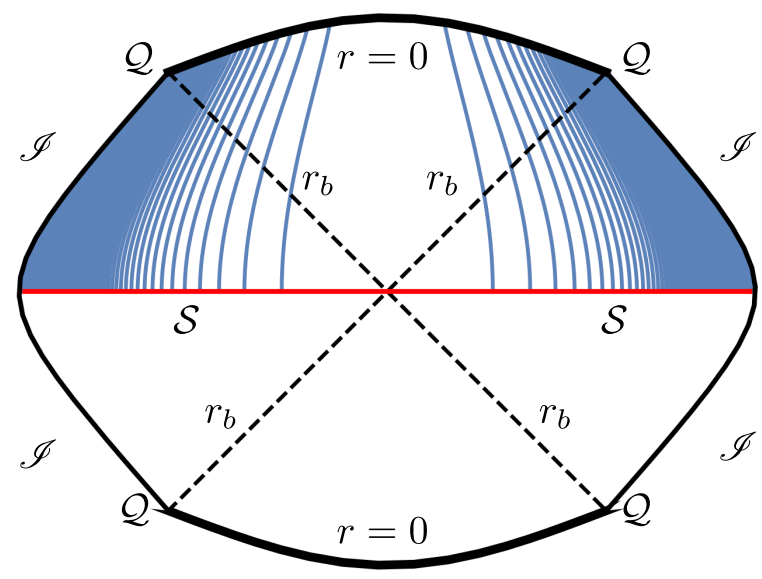

Figure 10: In this figure we show a numerical simulation of the congruence of conformal geodesics plotted in the Kruskal diagram of the Schwarzschild anti de Sitter solution for $M=28.56$ and $\beta$ given by (119). The graph also shows the initial data hypersurface $\mathcal{S}$, the singularity and the conformal boundaries.

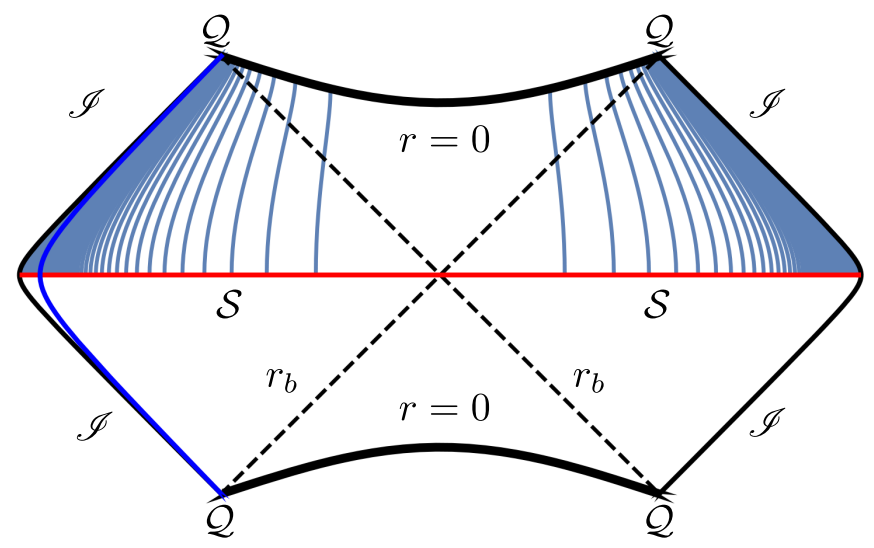

Figure 11: In this figure we show a numerical simulation of the congruence of conformal geodesics for the Schwarzschild anti de Sitter solution similar to the one presented in figure 10 but of a value of the mass parameter given by $M=0.34$. In this case the critical conformal geodesic is also plotted.

with initial conditions

$$
\begin{aligned}
& U_{*}=V_{*}=0, \\
& U_{*}^{\prime}=-\frac{\sqrt[4]{3 r_{b}^{2}+1}}{2 \sqrt{r_{b}}} \exp \left(\frac{\left(3 r_{b}^{2}+2\right)}{2 r_{b} \sqrt{3 r_{b}^{2}+4}} \arctan \left(\frac{3 r_{b}}{\sqrt{3 r_{b}^{2}+4}}\right)\right) .
\end{aligned}
$$

Equation 139c entails

$$
\frac{U^{\prime}}{\sin (U)}=\frac{V^{\prime}}{\sin (V)} .
$$

Combining the latter expression with the initial data for the congruence leads to

$$
U(t)=V(t) .
$$

We can write equation 139a as

$$
\frac{U^{\prime \prime}}{U^{\prime}}=\frac{2 U^{\prime}}{\sin (2 U)}\left(\cos (2 U)-\frac{r_{b}\left(2 r^{3}+r_{b}^{3}+r_{b}\right)}{r^{2}\left(3 r_{b}^{2}+1\right)}\right),
$$


from which one obtains

$$
\log \left(U^{\prime}\right)-\log \left(U_{*}^{\prime}\right)=\int_{0}^{U}\left(\frac{2 d s}{\sin (2 s)}\left(\cos (2 s)-\frac{r_{b}\left(2 r(s)^{3}+r_{b}^{3}+r_{b}\right)}{r(s)^{2}\left(3 r_{b}^{2}+1\right)}\right)\right) .
$$

In this integral, the function $r(s)$ is defined implicitly through the relation

$$
s=\arctan \left(\frac{\sqrt{\left|r(s)-r_{b}\right|}}{\sqrt[4]{r(s)^{2}+r(s) r_{b}+r_{b}^{2}+1}} \Delta\left(r(s), r_{b}\right)\right)
$$

obtained from equation (136) setting $U=V$. Exploiting the last equation one makes a change of variables in the integral of equation (141) to obtain

$$
\log \left(U^{\prime}\right)-\log \left(U_{*}^{\prime}\right)=\int_{r_{b}}^{r} \frac{N\left(r, r_{b}\right)}{D\left(r, r_{b}\right)} d r,
$$

where

$$
\begin{array}{r}
N\left(r, r_{b}\right) \equiv \sqrt{r^{2}+r r_{b}+r_{b}^{2}+1}\left(2 r^{2} r_{b}-r\left(r_{b}^{2}+1\right)-r_{b}\left(r_{b}^{2}+1\right)\right) \\
-\left(2 r^{3} r_{b}+r^{2}\left(3 r_{b}^{2}+1\right)+r_{b}^{4}+r_{b}^{2}\right) \Delta\left(r, r_{b}\right) \\
D\left(r, r_{b}\right) \equiv 2 r r_{b}\left(r^{2}+r r_{b}+r_{b}^{2}+1\right)^{\frac{3}{2}}\left(\frac{\left(r-r_{b}\right) \Delta\left(r, r_{b}\right)}{\sqrt{r^{2}+r r_{b}+r_{b}^{2}+1}}-1\right)
\end{array}
$$

The integral of equation (143) can be computed explicitly, and after lengthy algebra one obtains

$$
\frac{2 U^{\prime}}{\sin (2 U)}=-\frac{\left(3 r_{b}^{2}+1\right)^{\frac{7}{4}}}{2 r_{b}^{\frac{3}{2}}}\left(\frac{r}{\left(r_{b}-r\right)\left(r^{2}+r r_{b}+r_{b}^{2}+1\right)}\right)^{\frac{1}{2}} .
$$

One eliminates the variable $U$ in this equation using the previous formulae to get $U^{\prime}$ in terms of $r^{\prime}$. This computation then renders

$$
\frac{\left(3 r_{b}^{2}+1\right)^{-\frac{3}{4}} r_{b}^{\frac{1}{2}} r^{\prime}}{\left(\left(r_{b}-r\right)\left(r^{2}+r_{b} r+1+r_{b}^{2}\right)\right)^{\frac{1}{2}}}=1
$$

whose solution can be reduced to quadratures

$$
\tilde{\tau}=\left(3 r_{b}^{2}+1\right)^{-\frac{3}{4}} r_{b}^{\frac{1}{2}} \int_{r}^{r_{b}} \frac{\mathrm{d} s}{\left(\left(r_{b}-s\right)\left(s^{2}+r_{b} s+1+r_{b}^{2}\right)\right)^{\frac{1}{2}}} .
$$

The integral is convergent for any value of $r$ in the interval $\left[0, r_{b}\right]$. In particular it enables us to compute the value of the physical time $\tilde{\tau}_{\xi}$ at which this conformal geodesic reaches the singularity:

$$
\tilde{\tau}_{\xi}=\left(3 r_{b}^{2}+1\right)^{-\frac{3}{4}} r_{b}^{\frac{1}{2}} \int_{0}^{r_{b}} \frac{\mathrm{d} s}{\left(\left(r_{b}-s\right)\left(s^{2}+r_{b} s+1+r_{b}^{2}\right)\right)^{\frac{1}{2}}} .
$$

Remark 27. Summarising, the conformal geodesic starting at the bifurcation sphere reaches the singularity in a finite amount of (physical) proper time.

\subsubsection{Conformal geodesics at the conformal boundary}

An important property of the anti de Sitter spacetime is that the conformal boundary can be ruled by a congruence of conformal geodesics - see e.g. [4. In this Section we show that the congruence of conformal geodesics in the Schwarzschild-anti de Sitter spacetime considered in the previous Sections extends to the conformal boundary to include curves with a similar property. 
The approach to the construction of conformal geodesics followed in this article has been to solve the relevant equations in the physical spacetime - this strategy, however, cannot be followed to analyse conformal geodesics at the conformal boundary. In this case, one needs to formulate the conformal geodesic equations and their initial data in a conformal extension.

Start by considering the conformal factor $\Xi=1 / \sqrt{1+r^{2}}$ chosen earlier in this Section - see equation 117 — and let

$$
\begin{aligned}
\overline{\boldsymbol{g}} & =\Xi^{2} \tilde{\boldsymbol{g}} \\
& =\frac{r^{3}+r-M}{r\left(1+r^{2}\right)} \mathbf{d} t \otimes \mathbf{d} t-\frac{r}{\left(1+r^{2}\right)\left(r^{3}+r-M\right)} \mathbf{d} r \otimes \mathbf{d} r-\frac{r^{2}}{1+r^{2}} \boldsymbol{\sigma} .
\end{aligned}
$$

By introducing a new radial coordinate $z=1 / r$, the latter metric can be shown to extend smoothly to the conformal boundary defined by $z=0$. In particular, the (Lorentzian) induced metric $\boldsymbol{\ell}$ on $\mathscr{I}$ (i.e. at $z=0$ ) is given by

$$
\ell=\mathbf{d} t \otimes \mathbf{d} t-\boldsymbol{\sigma}
$$

the standard metric of $\mathbb{R} \times \mathbb{S}^{2}$ — the 3-dimensional Einstein cylinder. On $\left(\mathbb{R} \times \mathbb{S}^{2}, \ell\right)$ consider now the family of curves given by

$$
x(\mathrm{~s}) \equiv\left(\mathrm{s}, \underline{x}_{\star}\right), \quad \underline{x}_{\star} \in \mathbb{S}^{2}, \quad \mathrm{~s} \in \mathbb{R} .
$$

These curves have tangent given by $\boldsymbol{\partial}_{\tau}$ and can be readily shown to be geodesics of the metric $\ell$. As $\left(\mathbb{R} \times \mathbb{S}^{2}, \ell\right)$ is a (3-dimensional) Einstein space, then the curve given by equation 145 is, up to a reparametrisation, a conformal geodesic — see e.g. Lemma 5.2 in [17]. To find the reparametrisation $\tau=\tau(s)$ exhibiting the conformal geodesic character of the curve one follows the argument of the proof of that Lemma and consider a candidate 1-form

$$
\overline{\boldsymbol{\beta}}=\alpha(\tau) \mathbf{d s}, \quad \text { for some smooth function } \alpha(\tau) .
$$

The conformal geodesic equations $15 \mathrm{a})-15 \mathrm{~b}$ readily give that

$$
\ddot{\mathrm{s}}+\alpha \dot{\mathrm{s}}^{2}=0, \quad \dot{\alpha}=\frac{1}{2} \dot{\mathrm{s}}\left(\alpha^{2}+1\right),
$$

where $\cdot$ denotes differentiation with respect to $\tau$. These equations can be solved to give

$$
\mathrm{s}=2 \arctan \frac{1}{2} \tau, \quad \alpha=\frac{1}{2} \tau
$$

Now, a calculation readily yields $\langle\overline{\boldsymbol{\beta}}, \dot{\boldsymbol{x}}\rangle=\alpha \dot{\mathrm{s}}$. Accordingly, the conformal factor $\vartheta$ on $\mathbb{R} \times \mathbb{S}^{2}$ satisfying the condition $\vartheta^{2} \ell(\dot{\boldsymbol{x}}, \dot{\boldsymbol{x}})=1$ obeys the ordinary differential equation

$$
\dot{\vartheta}=\langle\overline{\boldsymbol{\beta}}, \dot{\boldsymbol{x}}\rangle \vartheta, \quad \vartheta_{\star}=1 .
$$

This differential equation can be solved to give

$$
\vartheta=1+\frac{1}{4} \tau^{2}
$$

so that, in particular, one has that

$$
\overline{\boldsymbol{\beta}}=\frac{1}{2} \tau \mathbf{d} t=\vartheta^{-1} \mathbf{d} \vartheta
$$

Using the conformal factor $\vartheta$ one obtains a different representative of the conformal class of conformal boundary of the spacetime -i.e. $\ell^{\prime} \equiv \vartheta^{2} \ell$ so that

$$
\ell^{\prime}=\mathbf{d} \tau \otimes \mathbf{d} \tau-\left(1+\frac{1}{4} \tau^{2}\right)^{2} \boldsymbol{\sigma}
$$


where the parameter $\tau$ has been introduced as new time coordinate. This representative of the conformal class $[\ell]$ can be regarded as canonical as in it, the parameter $\tau$ is the proper time of the curve.

Summarising the previous discussion, we have found that the pair $(x(\tau), \bar{\beta}(\tau))$ given by

$$
x(\tau)=\left(2 \arctan \frac{1}{2} \tau, \underline{x}_{\star}\right), \quad \overline{\boldsymbol{\beta}}(\tau)=\frac{2 \tau}{4+\tau^{2}} \mathbf{d} \tau, \quad \tau \in \mathbb{R}, \quad \underline{x}_{\star} \in \mathbb{S}^{2},
$$

are solutions to the $\bar{g}$-conformal geodesics. Observe that, in particular, as the curve and the 1-form are completely intrinsic to the conformal boundary, then $\langle\overline{\boldsymbol{\beta}}, \boldsymbol{\nu}\rangle=0$ where $\nu$ is the unit normal vector to the initial hypersurface.

Remark 28. This result is, in fact, a general property of anti de Sitter-like spacetimes: a conformal geodesic in an anti-de Sitter-like spacetime which passes through a point $p \in \mathscr{I}$, is tangent to $\mathscr{I}$ at $p$ and which satisfies $\left.\langle\boldsymbol{\beta}, \boldsymbol{\nu}\rangle\right|_{p}=-\Pi$ with $\boldsymbol{\nu}$ the unit normal to $\mathscr{I}$ and $\Pi$ the so-called Friedrich scalar of the conformal representation, remains in $\mathscr{I}$ and defines a conformal geodesic for the conformal structure of $\mathscr{I}$ - see e.g. [17, Lemma 17.1. We recall that the Friedrich scalar at a timelike (or spacelike) conformal boundary is closely related to the extrinsic curvature of the hypersurface - in particular, if $\Pi=0$ then the conformal boundary is extrinsically flat, see [17, Section 11.4.4. In this Section we show that the congruence of conformal geodesics for the Schwarzschild-anti de Sitter spacetime considered in the previous Sections can be extended to include curves on the conformal boundary with the above property.

Remark 29. Observe that as $\tau \rightarrow \pm \infty$ then $\mathrm{s} \rightarrow \pm \pi$. Thus, the parameter $\tau$ does not allow to exhaust the whole of the cylinder $\mathbb{R} \times \mathbb{S}^{2}$. This phenomenon is a 3-dimensional analogue of a similar observation for the (4-dimensional) anti de Sitter spacetime - see 4] also [17] Section 6.4.2.

Relation between the conformal geodesics on the conformal boundary and those in the bulk. Finally, we analyse the relation between the family of curves on the conformal boundary of the Schwarzschild-anti de Sitter spacetime and the conformal geodesics in the interior of the spacetime that have been constructed earlier. To do this, it is recalled that the conformal geodesic equations $(15 \mathrm{a})$ and $(15 \mathrm{~b})$ are conformally invariant under a rescaling $\overline{\boldsymbol{g}}=\Xi^{2} \tilde{\boldsymbol{g}}$ if the 1 -form $\boldsymbol{\beta}$ transforms as

$$
\overline{\boldsymbol{\beta}}=\boldsymbol{\beta}-\Xi^{-1} \mathbf{d} \Xi .
$$

Thus, the initial data for the $\overline{\boldsymbol{g}}$-conformal geodesic equations implied by the initial data for the $\tilde{\boldsymbol{g}}$-conformal geodesic equations in 118) satisfies

$$
\bar{\beta}_{\star}=0
$$

for all points in the conformal extension $\mathcal{S}$ of the (physical) initial hypersurface $\tilde{\mathcal{S}}$. Moreover, it can be readily verified that the curves in $(146)$ are orthogonal to $\mathcal{S}$. Thus, they are the limit of the family of conformal geodesics in the interior of the spacetime considered in this Section.

\subsection{Analysis of the conformal geodesic deviation equation}

In this Section we apply the formalism introduced in Section 3.4 to verify that the congruence of conformal geodesics in the Schwarzschild-anti de Sitter spacetime constructed in the previous Section is non-singular. Remarkably, the various classes of conformal geodesics in the Schwarzschild spacetime discussed in the previous Sections can be analysed simultaneously.

In the case of the Schwarzschild-anti de Sitter equation, equation (48) with the value of $\beta$ given by 118 takes the form

$$
\frac{\mathrm{d}^{2} \tilde{\omega}}{\mathrm{d} \tilde{\tau}^{2}}=\left(\frac{M\left(\left(1+r_{\star}^{2}\right)^{2}-r_{\star} r^{3}\right)-r^{3}\left(1+r_{\star}^{2}\right)}{\tilde{r}^{3}\left(1+r_{\star}^{2}\right)^{2}}\right) \tilde{\omega}+\frac{2 r_{\star}\left(1+r_{\star}^{2}\right)+M\left(3 r_{\star}^{2}-1\right)}{2 \rho_{\star}\left(1+r_{\star}^{2}\right)^{2}} .
$$


It is observed that since both $M$ and $r$ are positive, we have the inequality

$$
\frac{M\left(\left(1+r_{\star}^{2}\right)^{2}-r_{\star} r^{3}\right)-r^{3}\left(1+r_{\star}^{2}\right)}{r^{3}\left(1+r_{\star}^{2}\right)^{2}}=\frac{M}{r^{3}}-\frac{M r_{\star}}{\left(r_{\star}^{2}+1\right)^{2}}-\frac{1}{r_{\star}^{2}+1}>-\frac{M r_{\star}}{\left(r_{\star}^{2}+1\right)^{2}}-\frac{1}{r_{\star}^{2}+1} .
$$

Therefore, $\tilde{\omega}$ satisfies the differential inequality

$$
\frac{\mathrm{d}^{2} \tilde{\omega}}{\mathrm{d} \tilde{\tau}^{2}} \geq-\left(\frac{M r_{\star}}{\left(r_{\star}^{2}+1\right)^{2}}+\frac{1}{r_{\star}^{2}+1}\right) \omega+\frac{2 r_{\star}\left(1+r_{\star}^{2}\right)+M\left(3 r_{\star}^{2}-1\right)}{2 \rho_{\star}\left(1+r_{\star}^{2}\right)^{2}} .
$$

The latter implies that the scalars $\tilde{\omega}$ and $\omega$ satisfy

$$
\omega \geq \varpi, \quad \tilde{\omega} \geq \Theta \varpi,
$$

where $\varpi$ is the solution of

$$
\begin{aligned}
& \frac{\mathrm{d}^{2} \varpi}{\mathrm{d} \tilde{\tau}^{2}}=-\left(\frac{M r_{\star}}{\left(r_{\star}^{2}+1\right)^{2}}+\frac{1}{r_{\star}^{2}+1}\right) \varpi+\frac{2 r_{\star}\left(1+r_{\star}^{2}\right)+M\left(3 r_{\star}^{2}-1\right)}{2 \rho_{\star}\left(1+r_{\star}^{2}\right)^{2}}, \\
& \varpi\left(0, \rho_{\star}\right)=\frac{r_{\star}}{\rho_{\star}}, \quad \varpi^{\prime}\left(0, \rho_{\star}\right)=0 .
\end{aligned}
$$

This differential equation can be explicitly solved, the result being

$$
\varpi=\frac{M\left(3 r_{\star}^{2}-1\right)+2 r_{\star}\left(r_{\star}^{2}+1\right)}{2 \rho_{\star}\left(r_{\star}\left(M+r_{\star}\right)+1\right)}-\frac{M\left(r_{\star}^{2}-1\right)}{2 \rho_{\star}\left(r_{\star}\left(M+r_{\star}\right)+1\right)} \cos \left(\frac{\tilde{\tau} \sqrt{r_{\star}\left(M+r_{\star}\right)+1}}{r_{\star}^{2}+1}\right) .
$$

Thus, using that

$$
\tilde{\omega} \geq \varpi \geq \min (\varpi)=\frac{2 M r_{\star}^{2}+2 r_{\star}\left(r_{\star}^{2}+1\right)}{2 \rho_{\star}\left(r_{\star}\left(M+r_{\star}\right)+1\right)} .
$$

Since the congruence never reaches the conformal boundary, one concludes that the congruence does not form caustic points.

Remark 30. We stress that the previous analysis holds for the three types of conformal geodesics considered in the previous Section.

\subsection{Conformal Gaussian coordinates in the Schwarzschild-anti de Sitter spacetime}

In Remark 21 it has been observed that the $g$-proper time, $\tau$, of the curves of the congruence of conformal geodesics in the Schwarzschild-anti de Sitter spacetime discussed in the previous Section does not cover the whole span of the curves. Thus, it follows that for the Schwarzschild-anti de Sitter spacetime it is not possible to construct global systems of conformal Gaussian coordinates as it was the case in the cases of positive and vanishing Cosmological constant.

Notwithstanding the observation made in the previous paragraph, under Assumption 1, and using methods similar to those employed in Sections 4.5 and 5.5 it is still possible to show that the congruence covers the maximal extension of the conformal representation of the spacetime defined by the conformal factor $\Theta$ as given by equation (120). Taking advantage of the discrete symmetries of the spacetime, the discussion can be restricted to the range $r_{\star} \in\left[r_{b}, \infty\right)$. We omit the details. 


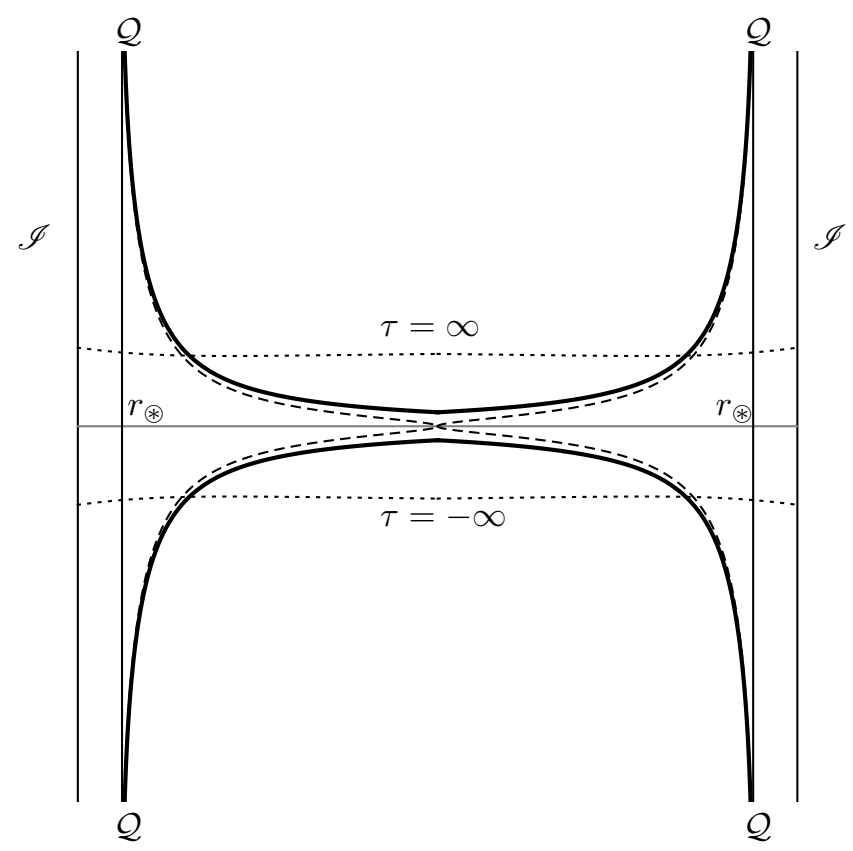

Figure 12: The Schwarzschild anti de Sitter spacetime in conformal Gaussian coordinates $\left(r_{\star}, \tilde{\tau}\right)$ for $M=1 / 2$. The horizontal central line represents the initial hypersurface $\tilde{\mathcal{S}}$ which is parametrised by $r_{\star}$. The vertical axis in this plot corresponds to the physical proper time $\tilde{\tau}$. In particular, the location of the horizon in conformal Gaussian coordinates, $\left(r_{b}, \tilde{\tau}\left(r_{b}, r_{\star}\right)\right)$ with $r_{b} \leq r_{\star} \leq r_{\circledast}$, corresponds to the dashed curve. The thick continuous line denotes the location of the singularity $\left(0, \tilde{\tau}\left(0, r_{\star}\right)\right)$ with $0 \leq r_{\star} \leq r_{\circledast}$. The inner vertical lines correspond to the conformal geodesic with initial datum $r_{\star}=r_{\circledast}$ while the outer vertical lines and the conformal boundary $\mathscr{I}$. The dotted horizontal lines shows boundary of the region that the congruence of conformal geodesics can cover with a single parametrisation of the unphysical proper time $\tau=\tau(\tilde{\tau})$. Similar to the case of the anti de Sitter spacetime the conformal boundary $\mathscr{I}$ cannot be covered with a single parametrisation of the unphysical proper time $\tau$.

\subsection{Summary}

The analysis of Section 6 can be summarised in the following Theorem:

Theorem 2 (Conformal geodesics in the Schwarzschild-anti de Sitter spacetime). For $r_{\star} \geq r_{b}$ let

$$
\tilde{\tau}_{\dagger}\left(r_{\star}\right) \equiv \min \left\{\frac{\pi\left(r_{\star}+1\right)}{\sqrt{r_{\star}^{2}+M r_{\star}+1}}, \tilde{\tau}_{\xi}\left(r_{\star}\right)\right\},
$$

where $\tilde{\tau}_{\xi}\left(r_{\star}\right)$ is defined by (127) if $r_{*}>r_{b}$ or (144) if $r_{*}=r_{b}$. The portion of the maximal extension of the Schwarzschild-anti de Sitter spacetime corresponding to the region

$$
\left\{\left(\tilde{\tau}, r_{\star}\right) \in\left(-\tilde{\tau}_{\dagger}\left(r_{\star}\right), \tilde{\tau}_{\dagger}\left(r_{\star}\right)\right) \times\left[r_{b}, \infty\right)\right\}
$$

can be covered by a non-singular congruence of conformal geodesics.

A qualitatively accurate depiction of the congruence of conformal geodesics in the Penrose diagram for various values of the mass parameter is shown in Figures 10 and 11. A qualitatively accurate depiction of the region of the spacetime that can be covered by conformal Gaussian coordinates can be found Figure 12 . 


\section{Concluding remarks}

In this article we have studied conformal geodesics in the Schwarzschild -de Sitter and Schwarzschildanti de Sitter families of spacetimes. In both cases, initial data for the congruence of curves can be chosen in such a manner that they cover the whole maximal extension of the spacetime. Moreover, in the case of the Schwarzschild-de Sitter spacetime, these curves provide global coordinate systems which, in turn, can be used as the starting point of a study of perturbations and global questions by means of conformal methods. To do this, one would need to study the exact solutions expressed in terms of the conformal Gaussian coordinates. As discussed in [8] for the Schwarzschild-de Sitter spacetime, this is not an easy task -in absence of an explicit change of coordinates one is forced to extract the required properties of the spacetime by means of a direct analysis of the evolution equations satisfied by the exact solution. In contrast in the Schwarzschild-anti de Sitter case only a portion of the maximal extension can be covered by conformal Gaussian coordinates. This phenomenon resembles the case of the anti de Sitter spacetime in which the conformal extension of the spacetime is only compact in the spatial directions but extends infinitely in the time direction. In this case, the unphysical proper time is exhausted before covering the full conformal extension of the spacetime.

\section{Acknowledgements}

AGP is supported by projects IT956-16 (Basque government, Spain), PTDC/MAT-ANA/1275/2014 ("Fundação para a Ciência e a Tecnología" (FCT), Portugal), FIS2014-57956-P ("Ministerio de Economía y Competitividad", Spain) and EG holds a scholarship (494039/218141) from Consejo Nacional de Ciencia y Tecnología (CONACyT). AGP wishes to thank the School of Mathematical Sciences of Queen Mary College, where part of this work was carried out, for hospitality. The three authors thank Dr. Christian Lübbe for enlightening discussions.

\section{References}

[1] S. L. Bażański \& V. Ferrari, Analytic extension of the Schwarzschild-de Sitter metric, Nuovo Cimento B 91, 126 (1986).

[2] P. Byrd \& M. Friedman, Handbook of Elliptic Integrals for Engineers and Physicists, Grundlehren der mathematischen Wissenschaften, Springer Verlag, (2013).

[3] L. Fidkowski, V. Hubeny, M. Kleban, \& S. Shenker, The black hole singularity in AdS/CFT, J. High E. Phys. 2, 014 (2004).

[4] H. Friedrich, Einstein equations and conformal structure: existence of anti-de Sitter-type space-times, J. Geom. Phys. 17, 125 (1995).

[5] H. Friedrich, Conformal Einstein evolution, in The conformal structure of spacetime: Geometry, Analysis, Numerics, edited by J. Frauendiener \& H. Friedrich, Lecture Notes in Physics, page 1, Springer, (2002).

[6] H. Friedrich, Conformal geodesics on vacuum spacetimes, Comm. Math. Phys. 235, 513 (2003).

[7] H. Friedrich \& B. Schmidt, Conformal geodesics in general relativity, Proc. Roy. Soc. Lond. A 414, 171 (1987).

[8] E. Gasperín \& J. A. Valiente Kroon, Perturbations of the asymptotic region of the Schwarzschild-de Sitter spacetime, Ann. H. Poincaré (2017).

[9] J. B. Griffiths \& J. Podolský, Exact space-times in Einstein's General Relativity, Cambridge University Press, (2009). 
[10] E. Hackmann \& C. Lämmerzahl, Geodesic equation in Schwarzschild-(anti)-de Sitter spacetimes: analytical solutions and applications, Phys. Rev. D 78, 024035 (2008).

[11] T. Klösch \& T. Strobl, Classical and quantum gravity in $1+1$ dimensions. Part II: the universal coverings, Class. Quantum Grav. 13(2395) (1996).

[12] D. F. Lawden, Elliptic functions and applications, Springer, (1989).

[13] C. Lübbe \& J. A. Valiente Kroon, On de Sitter-like and Minkowski-like spacetimes, Class. Quantum Grav. 26, 145012 (2009).

[14] C. Lübbe \& J. A. Valiente Kroon, A class of conformal curves in the Reissner-Nordström spacetime, Ann. Henri Poincaré 15, 1327 (2013).

[15] J. Podolský, The structure of the extreme Schwarzschild-de Sitter spacetime, Gen. Rel. Grav. 31, 1703 (1999).

[16] C. Stanciulescu, Spherically symmetric solutions of the vacuum Einstein field equations with positive cosmological constant, Master thesis, University of Vienna, (1998).

[17] J. A. Valiente Kroon, Conformal Methods in General Relativity, Cambridge University Press, (2016). 